\title{
Neuromusculoskeletal Dynamic Modelling of Human Movement in Motion Environments
}

by

\section{Burhanuddin Terai}

\author{
A thesis submitted to \\ the Faculty of Graduate and Postdoctoral Affairs \\ in partial fulfilment of \\ the requirements for the degree of \\ Master of Applied Science \\ in \\ Mechanical Engineering
}

Ottawa-Carleton Institute for Mechanical and Aerospace Engineering

Department of Mechanical and Aerospace Engineering

Carleton University

Ottawa, Ontario, Canada

December 2016

Copyright (C)

2016 - Burhanuddin Terai 


\section{Abstract}

Personnel onboard high-speed marine craft are exposed to eccentric slam impacts of up to $20 \mathrm{~g}$ due to hull separation from the water during operation. To maintain postural stability, occupants adopt a semi-squatted position to attenuate the highacceleration loading, which leads to severe acute and chronic musculoskeletal injuries, and hinders post-transit performance. The harsh environment warrants a thorough understanding of human-body behaviour in order to predict responses under specific conditions, and quantify energy expenditure in maintaining postural stability.

A comprehensive, three degree-of-freedom sagittal-plane musculoskeletal dynamic modelling framework was developed to estimate musculotendon forces from neuromuscular stimuli and joint kinematics to provide estimates of joint torques and muscle energetics. The model was calibrated and validated through experimental trials with seven participants in the laboratory and indicates good agreement with torque profiles obtained through inverse dynamics. The framework provides general applicability to postural stability in a wide range of motion environments and supports future investigation of injury criteria and occupant-seat interaction on high-speed craft. 
For Mom, Dad, Ibrahim, and Huseina. 


\section{Acknowledgments}

I would like to thank my co-supervisors Professor Fred Afagh and Professor Robert Langlois, for their continued motivation and guidance throughout my studies and for allowing me the freedom to explore without boundaries.

I want to thank my dear friends and lab mates at ADL and ABL. This path would not have been the same without their kind words of encouragement, stimulating discussions, and companionship on this journey of growth.

I'm grateful to NSERC and the MAE department for providing the financial support for this research, and the Research Ethics Board for facilitating the experimental trials to realize this work.

Last but not least, I'm deeply indebted to my family, whose vision and sacrifice has allowed me this opportunity, and to my dear wife, for her patience and unwavering support in this achievement. To you, I dedicate this thesis. 


\section{Table of Contents}

Abstract ii

Acknowledgments $\quad$ iv

$\begin{array}{ll}\text { Table of Contents } & \text { v }\end{array}$

List of Tables $\quad$ ix

List of Figures $\quad$ X

List of Acronyms xiv

List of Symbols $\quad$ xviii

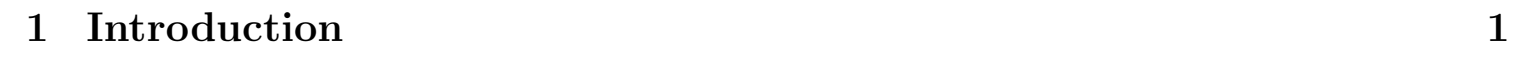

1.1 Background . . . . . . . . . . . . . . . . . . 2

1.1.1 Human Factors in the HSC Environment . . . . . . . . . . . . 2

1.1 .2 Modelling Standards . . . . . . . . . . . . . . . . . . 7

1.2 Literature Review . . . . . . . . . . . . . . . . . . . . . 9

$1.2 .1 \quad$ Human-body Dynamic Modelling . . . . . . . . . . . . . . . . 9

1.2 .2 Muscle Anatomy and Physiology . . . . . . . . . . . . . . . . 12

1.2 .3 Muscle Models . . . . . . . . . . . . . . . . . . . . 15

1.2 .4 Simulation . . . . . . . . . . . . . . . . . . . . 19 
1.2 .5 Summary . . . . . . . . . . . . . . . . . . . . . 21

1.3 Motivation and Scope of Research . . . . . . . . . . . . . . . . . 21

1.4 Thesis Overview . . . . . . . . . . . . . . . . . . . . . . . . . . 22

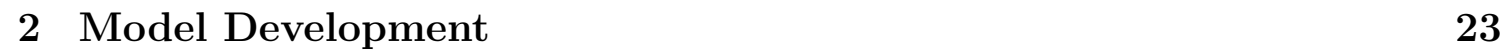

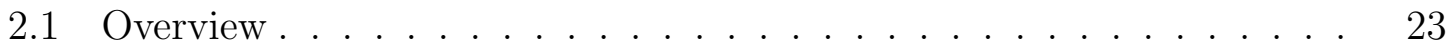

$2.1 .1 \quad$ Model Description . . . . . . . . . . . . . . . . . . 25

2.2 Muscle Activation Dynamics . . . . . . . . . . . . . . . . . . . 28

2.2 .1 Twitch Response . . . . . . . . . . . . . . . . . . . . 28

2.2 .2 Activation Model . . . . . . . . . . . . . . . . . . . . . 29

2.3 Musculotendon Contraction Dynamics _... . . . . . . . . . 30

2.3 .1 Muscle Passive Force-length Relationship . . . . . . . . . . 34

$2.3 .2 \quad$ Muscle Active Force-length Relationship . . . . . . . . . . 35

2.3 .3 Muscle Force-velocity Relationship . . . . . . . . . . . . . 35

2.3 .4 Muscle Pennation Angle $\ldots \ldots \ldots$. . . . . . . . . . . 36

2.3 .5 Tendon Model . . . . . . . . . . . . . . . . . . . . . . . 37

$2.3 .6 \quad$ Rigid-tendon Simplification $\ldots \ldots \ldots$

$2.4 \quad$ Energy Expenditure Model . . . . . . . . . . . . . . . . . . . . . 40

2.4 .1 Activation and Maintenance Heat Rate . . . . . . . . . . . 42

2.4 .2 Shortening and Lengthening Heat Rate . . . . . . . . . . 42

$2.4 .3 \quad$ Scaling Factors $\ldots \ldots \ldots \ldots$. . . . . . . . . . . . 44

2.4 .4 Total Energy Rate . . . . . . . . . . . . . . . . . . 45

$2.5 \quad$ Musculoskeletal Geometry $\ldots \ldots \ldots \ldots$

$2.6 \quad$ Rigid Body Dynamic Equations of Motion $\ldots \ldots \ldots \ldots$

$2.6 .1 \quad$ Model Description $\ldots \ldots \ldots \ldots$

2.6 .2 Joint Positions and Velocities . . . . . . . . . . . . . . 51

$2.6 .3 \quad$ Kinetic and Potential Energy $\ldots \ldots \ldots \ldots \ldots$ 
2.6 .4 Lagrange's Equations of Motion . . . . . . . . . . . . . . . 54

2.7 Chapter Summary . . . . . . . . . . . . . . . . . 55

$\begin{array}{lll}3 & \text { Experiment Design and Instrumentation } & 56\end{array}$

3.1 Overview . . . . . . . . . . . . . . . . . . . 56

3.2 Anthropometric Parameters . . . . . . . . . . . . . . . . . 58

3.3 Motion Platform . . . . . . . . . . . . . . . . . . . . . . 60

3.3 .1 Post-processing Accelerations . . . . . . . . . . . . . . . 64

3.4 Motion Capture . . . . . . . . . . . . . . . . . . . . . . . 64

$3.4 .1 \quad$ Post-processing Joint Kinematics . . . . . . . . . . . . . . . . 67

3.5 Electromyography $\ldots \ldots \ldots$. . . . . . . . . . . . . 70

3.5.1 Post-processing Muscle Activations . . . . . . . . . . . . . . . 73

$3.6 \quad$ Energy Expenditure . . . . . . . . . . . . . . . . . . . . . 74

3.7 Data Collection Procedure . . . . . . . . . . . . . . . . . . . . . . 78

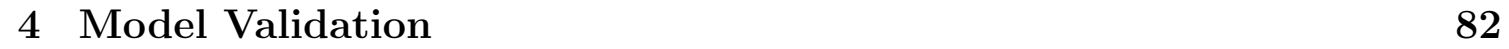

4.1 Model Calibration . . . . . . . . . . . . . . . . . . . . . . . . . . . . 82

4.1 .1 Parameter Tuning . . . . . . . . . . . . . . . . . 83

4.2 Results . . . . . . . . . . . . . . . . . . . . . . . . . . . . . . . . . . . . . . 88

$4.2 .1 \quad$ Chirp Motion Profiles . . . . . . . . . . . . . . . . . . . . . . . 88

4.2 .2 3DOF Motion Profiles . . . . . . . . . . . . . . . . . . 94

4.3 Discussion . . . . . . . . . . . . . . . . . . . . . . . . . . . . . . . . . 97

$4.3 .1 \quad$ Modelling Assumptions . . . . . . . . . . . . . . . . . . . . . . 97

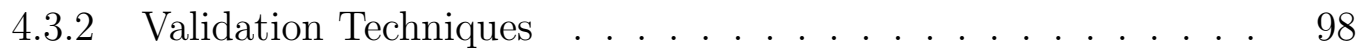

$4.3 .3 \quad$ Calibration and Parameter Tuning . . . . . . . . . . . . . . . 99

4.3 .4 Rigid-tendon Simplification . . . . . . . . . . . . . . . . 100

4.3 .5 Implications of the MTM Model . . . . . . . . . . . . . . . . . 101

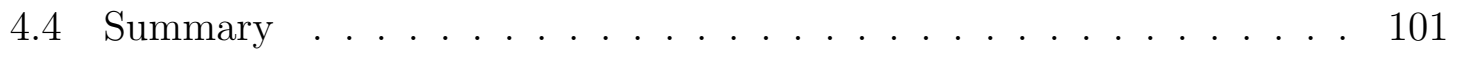


\begin{tabular}{|lll}
\hline 5 & Conclusions and Future Work & 103
\end{tabular}

$5.1 \quad$ Model Development . . . . . . . . . . . . . . . . . . . . . . . 103

5.2 Experiment Design and Instrumentation . . . . . . . . . . . . 104

5.3 Model Validation . . . . . . . . . . . . . . . . . . . . . . . . 105

5.4 Future Work . . . . . . . . . . . . . . . . . . . . . . . . 105

5.4 .1 Optimal Controller . . . . . . . . . . . . . . . . . 106

$5.4 .2 \quad$ Muscle Fatigue . . . . . . . . . . . . . . . . 106

5.4 .3 Model Fidelity . . . . . . . . . . . . . . . . . 107

5.4 .4 Summary $\ldots \ldots \ldots \ldots \ldots \ldots$

\begin{tabular}{ll}
\hline References & 109
\end{tabular}

\begin{tabular}{ll}
\hline Appendix A Ethics Clearance & 115
\end{tabular}

\begin{tabular}{|lll}
\hline Appendix B Sample Joint Torque Profiles & 117
\end{tabular} 


\section{List of Tables}

2.1 Musculotendon parameters for seven major muscles of the lower extremity. Fibre composition taken from Umberger, all other values taken from OpenSim. . . . . . . . . . . . . . . . . 34

2.2 Musculotendon geometry model, simplified from Menegaldo. Moment arms reflect generalized coordinates in the order they appear; coefficients listed are as shown in their respective equations. . . . . . . . . 49

2.3 Inertial parameters of three-link biodynamic model. . . . . . . . . . . 51

$3.1 \quad$ Experimental parameters for model validation. . . . . . . . . . . . . . 57

3.2 Measured and computed anthropometric parameters. . . . . . . . . . 58

$3.3 \quad$ MOOG platform software limits for degrees of freedom. . . . . . . . . 61

3.4 Motion profiles selected for experimental trials. . . . . . . . . . . 62

3.5 Electrode placements for the seven major lower-extremity muscles investigated in this study. . . . . . . . . . . . . . . . . . . 71

$3.6 \quad$ Relationship between RER, energy, and food source. . . . . . . . . . 77

4.1 Tuned maximum isometric muscle forces for seven participants with nominal values taken from OpenSim representing averages from numerous investigators. . . . . . . . . . . . . . . . . . . 87

4.2 Absolute and normalized RMSE of joint torque model predictions during combined 3DOF motion profile representative of frigate deck motion. 97 


\section{List of Figures}

1.1 Hull separation of an HSC during a wave impact. . . . . . . . . . . . 3

1.2 Ullman Dynamics Atlantic suspension seat with full restraint harness

(left) and differences in occupant posture on HSC seats (right). . . . . 4

1.3 Potential effects of lateral supports on spine deflection. . . . . . . . . 4

$1.4 \quad$ HSC operator in seated posture with restricted line of sight (top); and

semi-squatted posture over the seat pan with increased line of sight

(bottom). . . . . . . . . . . . . . . 6

1.5 Spatial coordinate frame defining biomechanical movement (left); and classical four-mass sagittal-plane inverted pendulum model for postural stability analysis (right). . . . . . . . . . . . . . . . . . . . . . . 10

$1.6 \quad$ Organization of skeletal muscle structure illustrating the sliding filament model facilitating muscle contraction. . . . . . . . . . . . . . . 14

1.7 Hill's lumped parameter model describing the dynamics of muscle contraction. . . . . . . . . . . . . . . . . . 18

1.8 Biarticular muscles (left to right): gastrocnemii, biceps femoris, and rectus femoris shown with the respective joint's moment arms. . . . . 19

2.1 Neuromusculoskeletal modelling process. . . . . . . . . . . . . . . . 24

2.2 Four-segment musculoskeletal model of foot, leg, thigh, and HAT actuated by seven major force-producing muscles about ankle, knee, and hip, with accurate depiction of muscle origin and insertion sites on bone. 27 
2.3 Hill-type musculotendon model describing contraction dynamics. (a) Active and passive force-length relationships of muscle; (b) Active force-velocity relationship of muscle; (c) Tendon force-strain relationship. . . . . . . . . . . . . . . . . . . . . . 31

2.4 Simplified geometric representation of tendon and muscle fibres at pennation angle $\alpha$ for musculotendon modelling. . . . . . . . . . . . . . . 37

2.5 Delp's lower extremity model (left) and sagittal-plane knee geometry model for determining kinematics and moment arms (right). . . . . . 46

2.6 Three-link inverted pendulum model of leg, thigh, and HAT rotating about ankle, knee, and hip joints, respectively, under prescribed platform motions $\dot{x}, \dot{y}$, and $\dot{\theta}_{s} . \ldots \ldots \ldots$. . . . . . . . . . 50

$3.1 \quad$ Experimental setup illustrating instrumentation and the 3DOF motions in surge, heave, and pitch corresponding to the $x, y$, and $\theta_{s}$ directions shown. . . . . . . . . . . . . . . . 57

3.2 Palpable bony prominences in the lower extremity used to define segment lengths and locate muscle origin and insertion points. SENIAMrecommended ideal muscle belly locations for electrode placements are identified by circular markers. . . . . . . . . . . . . . . . . . . . . . . 59

3.3 MOOG 6DOF-2000E Gough-Stewart platform. . . . . . . . . . . . . . 60

$3.4 \quad$ A sample of surge chirp (top) and combined 3DOF (bottom) motion

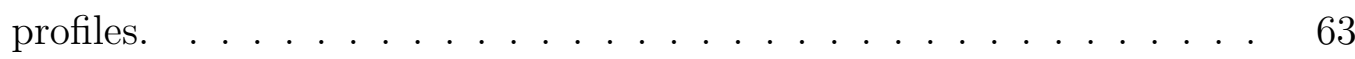

$3.5 \quad$ OptiTrack rigid-body skeleton model fit to participant's T-pose illustrating reflective marker placements and corresponding rigid-body limb segments. . . . . . . . . . . . . . . 66

3.6 The 23 major joint centres defining the BVH skeleton (left), and the resolved planar inverted pendulum model (right). . . . . . . . . . . . 68 
3.7 Joint angle conventions of the (left to right) musculoskeletal geometry model, BVH model, and 3IP dynamic model used in data post-processing. 69

3.8 Active bar electrodes used with the Delsys Bagnoli EMG system. . . 72

3.9 Processing of raw EMG signal. . . . . . . . . . . . . . . . . 75

3.10 Breath by breath metabolic analyzer system setup. . . . . . . . . . . 79

3.11 Foot stomp signatures in raw data evident from knee angle and knee extensor activations. . . . . . . . . . . . . . . . . . . . . 81

4.1 Calibrated and uncalibrated joint torques comparison of MTM and 3IP models during body-weight squats. . . . . . . . . . . . . . . . . . 85

4.2 Joint kinematics, muscle activations and musculotendon forces during a body-weight squat. . . . . . . . . . . . . . 86

$4.3 \quad$ Muscle activations and joint kinematics illustrating coping mechanisms during a surge chirp profile. . . . . . . . . . . . . . . . . . . . 89

4.4 Joint torques from MTM muscle model and 3IP inverse dynamics during a surge chirp profile. . . . . . . . . . . . . . . . . . . . . . . . . . 91

$4.5 \quad$ MTM and 3IP model energetics compared with total body energy expenditure measured with the BBB1LP analyzer during a surge chirp profile (top); a magnified view detailing the MTM and 3IP results (bottom). . . . . . . . . . . . . . . . . . 93

4.6 Joint torques from MTM muscle model and 3IP inverse dynamics during a combined 3DOF motion profile. . . . . . . . . . . . . . . . 95

4.7 MTM and 3IP model energetics compared with total body energy expenditure measured with the BBB1LP analyzer during a combined 3DOF motion profile (top); a magnified view detailing the MTM and 3IP results (bottom). . . . . . . . . . . . . . . . 96

5.1 Schematic of the three-compartment model illustrating transition of muscle fibre states. . . . . . . . . . . . . . . . . . . . . . . . 107 
B.1 Subject 1 joint torques from MTM muscle model and 3IP inverse dynamics during a combined 3DOF motion profile. . . . . . . . . . . . . 118

B.2 Subject 2 joint torques from MTM muscle model and 3IP inverse dynamics during a combined 3DOF motion profile. . . . . . . . . . . . . 119

B.3 Subject 3 joint torques from MTM muscle model and 3IP inverse dynamics during a surge chirp profile. . . . . . . . . . . . . . . . . . . . 120

B.4 Subject 4 joint torques from MTM muscle model and 3IP inverse dynamics during a combined 3DOF motion profile. . . . . . . . . . . . . 121

B.5 Subject 5 joint torques from MTM muscle model and 3IP inverse dynamics during a combined 3DOF motion profile. . . . . . . . . . . . . 122

B.6 Subject 6 joint torques from MTM muscle model and 3IP inverse dynamics during a combined 3DOF motion profile. . . . . . . . . . . . . 123

B.7 Subject 7 joint torques from MTM muscle model and 3IP inverse dynamics during a combined 3DOF motion profile. . . . . . . . . . . . . 124 


\section{List of Acronyms}

\begin{tabular}{ll} 
Acronyms & Definition \\
\hline \hline $2 \mathrm{D}$ & two-dimensional \\
$3 \mathrm{D}$ & three-dimensional \\
$\mathrm{AM}$ & activation and maintenance \\
$\mathrm{ATP}$ & adenosine triphosphate \\
$\mathrm{BF}$ & biceps femoris \\
$\mathrm{BMI}$ & body mass index \\
$\mathrm{BVH}$ & Biovision hierarchy \\
$\mathrm{CE}$ & contractile element \\
$\mathrm{CNS}$ & central nervous system \\
$\mathrm{COM}$ & carbon dioxide \\
& \\
$\mathrm{CH}$ & degree of freedom \\
&
\end{tabular}




\begin{tabular}{|c|c|}
\hline DRI & dynamic response index \\
\hline EMG & electromyography \\
\hline EMI & electromagnetic interference \\
\hline F-L & force-length \\
\hline $\mathrm{FT}$ & fast-twitch \\
\hline $\mathrm{F}-\mathrm{V}$ & force-velocity \\
\hline GAS & gastrocnemius \\
\hline GLU & glutei \\
\hline GM & gluteus medius \\
\hline HAM & hamstrings \\
\hline HAT & head, arms, and trunk \\
\hline $\mathrm{HSC}$ & high-speed craft \\
\hline IED & inter-electrode distance \\
\hline ISO & International Organization for Standardization \\
\hline MADYMO & mathematical dynamic model \\
\hline MIF & motion-induced fatigue \\
\hline MT & musculotendon \\
\hline MVC & maximum voluntary contraction \\
\hline $\mathrm{O}_{2}$ & oxygen \\
\hline
\end{tabular}




\begin{tabular}{|c|c|}
\hline ODE & ordinary differential equation \\
\hline PCSA & physiological cross-sectional area \\
\hline $\mathrm{PE}$ & parallel elastic \\
\hline RER & respiratory exchange ratio \\
\hline $\mathrm{RF}$ & rectus femoris \\
\hline RMS & root mean square \\
\hline RMSE & root mean square error \\
\hline RQ & respiratory quotient \\
\hline RQI & ride quality index \\
\hline SDOF & single degree of freedom \\
\hline $\mathrm{SE}$ & series elastic \\
\hline$S_{\text {ed }}$ & equivalent static compressive stress dose \\
\hline sEMG & surface electromyography \\
\hline SENIAM & surface electromyography for the non-invasive assessment of muscles \\
\hline SL & shortening and lengthening \\
\hline SOL & soleus \\
\hline $\mathrm{ST}$ & slow-twitch \\
\hline $\mathrm{TA}$ & tibialis anterior \\
\hline VAS & vasti \\
\hline
\end{tabular}


VDV vibration dose value

VM vastus medialis 


\section{List of Symbols}

\section{Symbols Definition}

\begin{tabular}{ll}
\hline \hline$\alpha^{M}$ & muscle fibre pennation angle \\
$\alpha_{o}^{M}$ & muscle fibre pennation angle at optimal muscle fibre length \\
$\alpha_{L}$ & lengthening heat rate coefficient \\
$\alpha_{S(S T)}$ & shortening heat rate coefficient for slow-twitch fibres \\
$\alpha_{S(F T)}$ & shortening heat rate coefficient for fast-twitch fibres \\
$\alpha_{t}$ & muscle twitch response gain coefficient \\
$\beta_{i}$ & muscle twitch response recursive coefficient \\
$\gamma_{i}$ & muscle twitch response filter coefficient \\
$\gamma_{L}$ & active force-length relationship shape factor \\
$\varepsilon_{j}$ & muscle physiological cross-sectional area \\
&
\end{tabular}




\begin{tabular}{|c|c|}
\hline$\varepsilon_{\text {toe }}^{T}$ & tendon strain in linear region \\
\hline$\varepsilon_{0}^{T}$ & tendon strain due to maximum isometric force \\
\hline$\eta$ & actin-myosin cross-bridges per unit volume \\
\hline$\kappa$ & spring constant for cross-link between actin and myosin \\
\hline$\lambda$ & distance between successive cross-bridge binding sites \\
\hline$\mu_{F T}$ & proportion of fast-twitch muscle fibres \\
\hline$\rho^{M}$ & muscle density \\
\hline$\sigma^{M}$ & specific tension of muscle \\
\hline$\tau_{a}$ & activation dynamics combined time constant \\
\hline$\tau_{\text {act }}$ & activation time constant \\
\hline$\tau_{\text {deact }}$ & deactivation time constant \\
\hline$\omega_{0}$ & frequency increment \\
\hline$\xi$ & muscle activation scaling factor for heat rate of muscle \\
\hline$\zeta$ & $\begin{array}{l}\text { aerobic/anaerobic metabolic pathway scaling factor for } \\
\text { heat rate of muscle }\end{array}$ \\
\hline$a$ & muscle activation \\
\hline$A$ & amplitude \\
\hline$e$ & surface electromyography envelope \\
\hline$\dot{E}$ & energy expenditure rate \\
\hline
\end{tabular}




\begin{tabular}{|c|c|}
\hline$f_{0}$ & starting frequency \\
\hline$f_{L}$ & muscle force-length relationship \\
\hline$f_{V}$ & muscle force-velocity relationship \\
\hline$F$ & force \\
\hline$F^{M}$ & total muscle force \\
\hline$F_{a}^{M}$ & active muscle force \\
\hline $\bar{F}_{l e n}^{M}$ & maximum normalized muscle force during lengthening \\
\hline$F^{P E}$ & parallel (passive) elastic muscle force \\
\hline$F_{\text {iso }}^{\max }$ & maximum isometric muscle force \\
\hline$F^{T}$ & tendon force \\
\hline $\bar{F}_{\text {toe }}^{T}$ & normalized tendon force in nonlinear toe region \\
\hline$G$ & activation dynamics exponential linearity parameter \\
\hline$H$ & body height \\
\hline$h^{M}$ & muscle height \\
\hline$\dot{H}$ & muscle heat generation rate \\
\hline$\dot{h}$ & muscle heat generation rate per unit muscle mass \\
\hline$\dot{h}_{A M}$ & activation and maintenance heat generation rate per unit \\
\hline
\end{tabular}




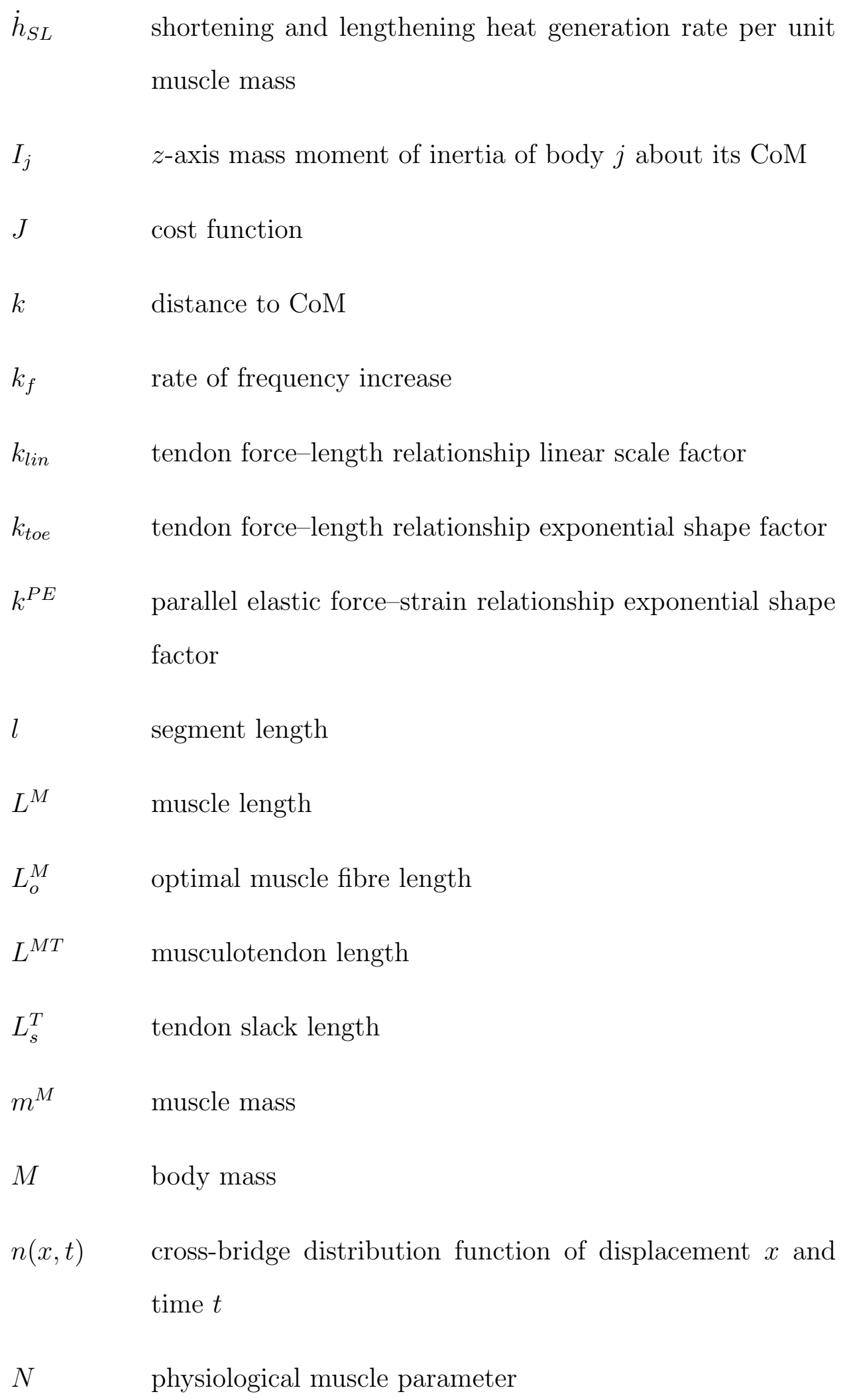




$\begin{array}{ll}p & \text { integer exponent for objective cost function } \\ \left\{\vec{r}_{A}\right\}^{l} & \text { position vector of point } A \text { in local reference frame } \\ r^{M} & \text { muscle moment arm } \\ r_{g} & \text { radius of gyration } \\ s(t) & \text { sarcomere length } \\ S_{f} & \text { active muscle force-velocity relationship shape factor } \\ T & \text { scalar joint torque } \\ u & \text { neural excitation } \\ \dot{W}^{M} & \text { velocity vector of point } A \text { in global reference frame } \\ \left.V_{A}\right\}^{g} & \text { musculotendon velocity } \\ V_{\text {max }}^{M} & \text { volume of CO }{ }_{2} \text { produced } \\ V^{M} & \text { muscle contraction velocity } \\ & \end{array}$




\section{Chapter 1}

\section{Introduction}

Personnel onboard high-speed marine craft (HSC) are exposed to eccentric slam impacts due to hull separation from the water during operation. As a result, occupants are subjected to repeated, high-acceleration impacts which lead to severe acute and chronic musculoskeletal injuries, and hinder their performance during and posttransit. While commercially-available suspension seats have the potential to reduce the harsh environmental effects on individual health and safety, the performance requirements to provide suitable shock mitigation improvements are not well understood. As a result, occupants often adopt a semi-squatted position over the seat pan to maintain postural stability and attenuate shock loading, placing them at direct risk for severe fatigue and injury in the lower extremity. The harsh environments of HSC warrant a thorough understanding of the behaviour of the human body in order to predict responses under specific conditions, as well as energy expenditure in maintaining postural stability. Doing so has the potential to reduce the risk of injury through efficient seat and hull designs and improve operational planning for mission efficacy.

The modelling framework introduced in this study uses neuromuscular stimuli to predict kinematics and kinetics of the human body actively with muscle engagement, 
and estimate energy expenditure in doing so. While motivated by the HSC environment, the framework has general applicability to postural stability in a wide range of motion environments be it land, sea, or air. A modelling approach has the benefits of reducing cost - both health and monetary, and can directly be applied under a wide range of input conditions to predict the risk of injury, investigate occupant-seat interaction, and for operational planning.

This chapter provides background information on human factors considerations in the HSC environment and current modelling standards in Section 1.1, an investigation of the state-of-the-art of human-body dynamic modelling capabilities in Section 1.2 , and finally outlines the research scope and overview of the thesis in Sections 1.3 and 1.4, respectively.

\section{$1.1 \quad$ Background}

\subsubsection{Human Factors in the HSC Environment}

High-speed craft are small boats designed to deliver personnel and equipment at high speeds in low to moderate sea conditions. These vessels are primarily used in military applications for patrol, search and rescue, and other missions where passengers support heavy operational loads from clothing and equipment worn and manual handling of heavy items, and are required to immediately perform high-intensity, physicallydemanding tasks post-transit. Exceeding speeds of 40 knots, the hull constantly experiences vertical shocks of up to $10 \mathrm{~g}$, occasionally reaching $20 \mathrm{~g}$, as a result of hull separation from the water, as shown in Figure 1.1. These shocks are potentially higher than those a jet pilot experiences upon ejection [1,2]. As a result, HSC transits are among the harshest operating environments as compared to any other mode of transport. In addition to the primary vertical loading, occupants are subjected 
to lateral, longitudinal, and torsional loading resulting in a host of severe acute and chronic musculoskeletal injuries, motion-induced fatigue (MIF), and reduced situational awareness [2]. Over $65 \%$ of boat operators have reported at least one injury; with the most common being in the lower back, knee, shoulder, neck, upper back, and ankle, as well as serious trauma, vertebral fractures, and intervertebral disc injuries in some cases [3]. A variety of commercially-available suspension seats, such as
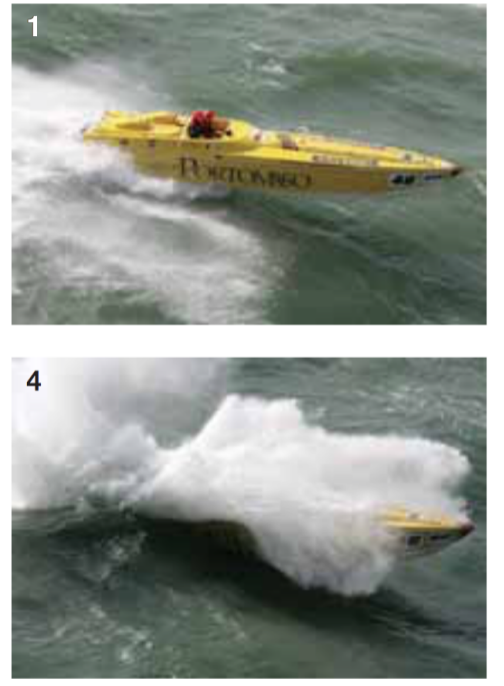
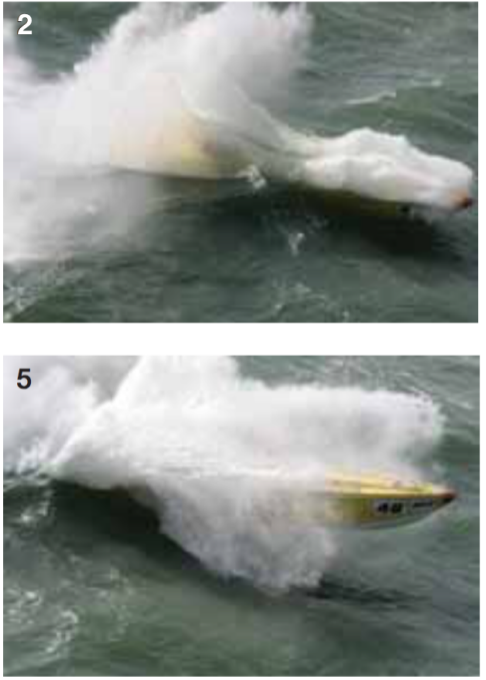
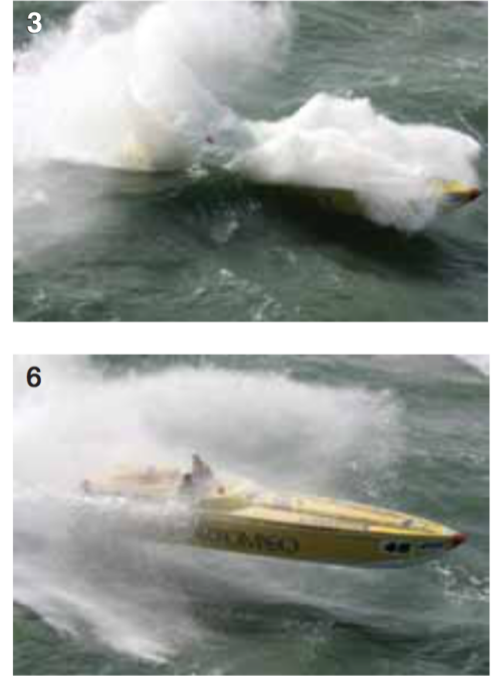

Figure 1.1: Hull separation of an HSC during a wave impact 2].

the Ullman Dynamics Atlantic, shown in Figure 1.2, are used to mitigate the shock loading. The stiffness and damping characteristics of these seats can often be tuned to suit operational conditions. However, the performance requirements to establish compliance with sea conditions in order to provide sufficient improvements to health and safety are not well understood [4].

Improperly-designed seats are potentially more dangerous to occupants. While seat restraints can give a sense of security, over-constrained supports can induce stress concentrations along the spine during a lateral impact. As shown in Figure 1.3 , shoulder and torso supports can cause excessive deflections in the neck rather than distributing it across the length of the spine. Similarly, seat cushions act as me- 

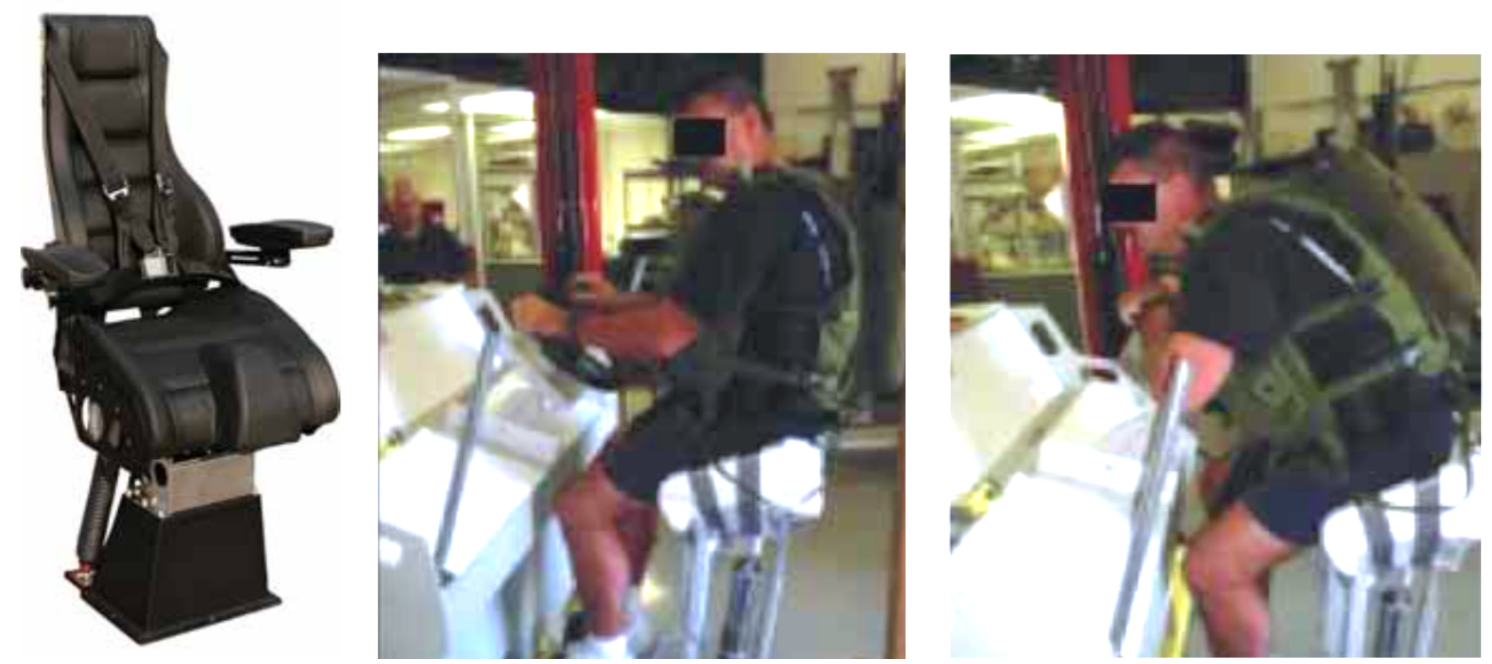

Figure 1.2: Ullman Dynamics Atlantic suspension seat with full restraint harness (left) and differences in occupant posture on HSC seats (right) [2].
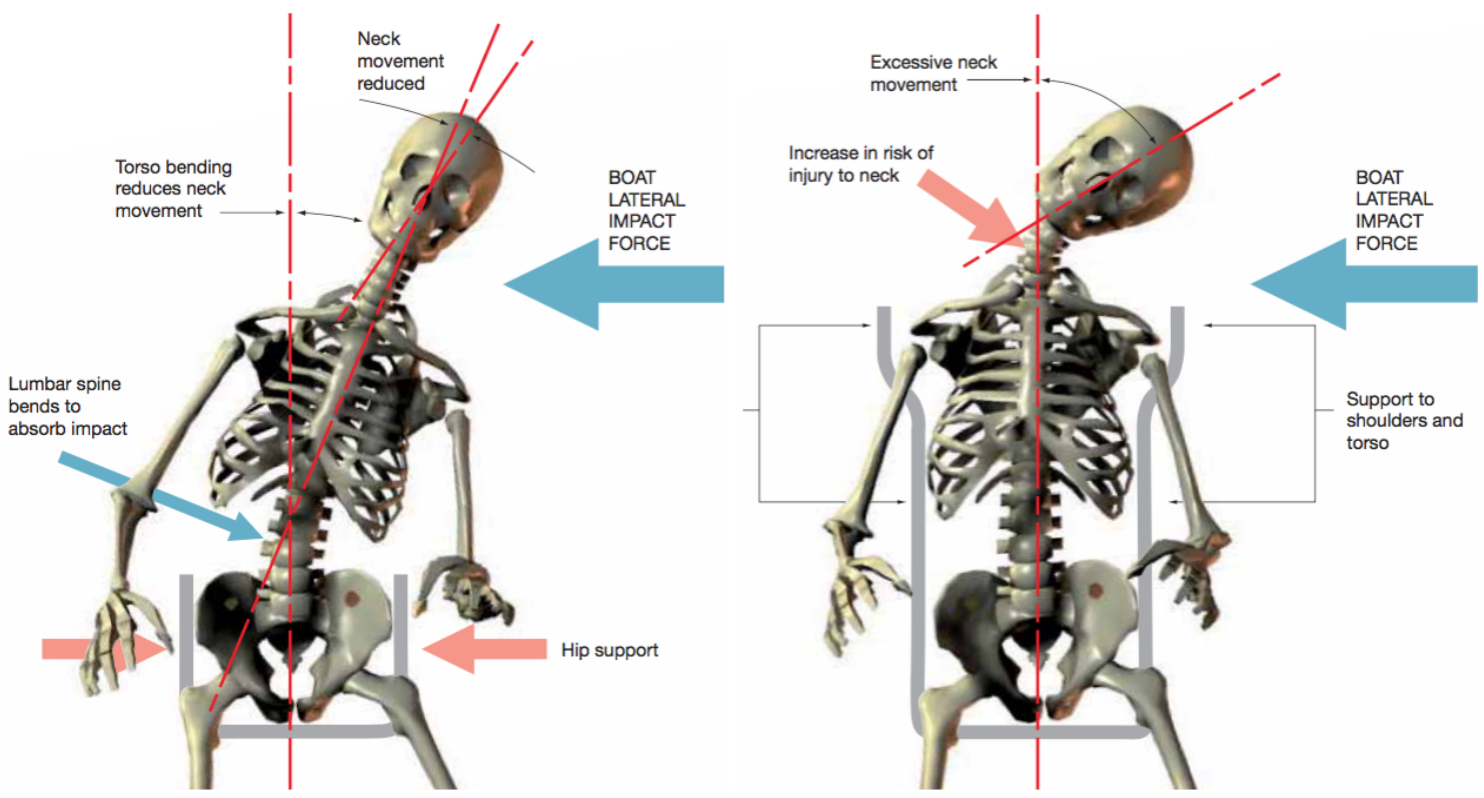

Figure 1.3: Potential effects of lateral supports on spine deflection [2]. 
chanical filters to dampen shock loading by providing the necessary displacement for deceleration. If the cushion is too soft, however, the foam can collapse during a large impact, causing it to bottom out and thus failing to attenuate the magnitude of the impact on the occupant properly. The spine has the ability to absorb significant impact when force is applied longitudinally. When misaligned due to poor posture, the shear component of the force vector on the spine induces transverse loads between vertebrae, significantly reducing the force required to cause injury. Many of the commercially-available seats are not designed for ergonomics, forcing occupants to take on poor postures during operation which places them at an increased risk during an impact, as shown in Figure 1.2, and they have been found to be restrictive in line of sight [2], as shown in Figure 1.4.

In the interest of comfort, passengers opt to brace themselves in a semi-squatted position over the seat pan, as shown in Figure 1.4, to maintain postural stability and attenuate impacts with their legs. To do so, the lower extremity muscles contract eccentrically (direction of force opposes motion) to decelerate the induced motion. These rapid eccentric muscle contractions cause localized fatigue and muscle damage indicated by elevated creatine kinase levels up to 72 hours post-transit, which is consistent with self-reported pain and soreness in occupants. Creatine kinase is a muscle enzyme involved in energy production that is commonly assayed in blood tests for indications of tissue damage [1,2]. The result is a significant reduction in physical performance: decreased jump height, running capability, and power output. On some occasions, this has also been known to cause hematuria (blood in the urine) an indication of vigorous exercise [1]. Therefore, while not considered a highly-aerobic activity, the physical work associated with mitigating shocks during an HSC transit causes motion-induced fatigue from the elevated energy expenditure and localized muscle damage [2]. 

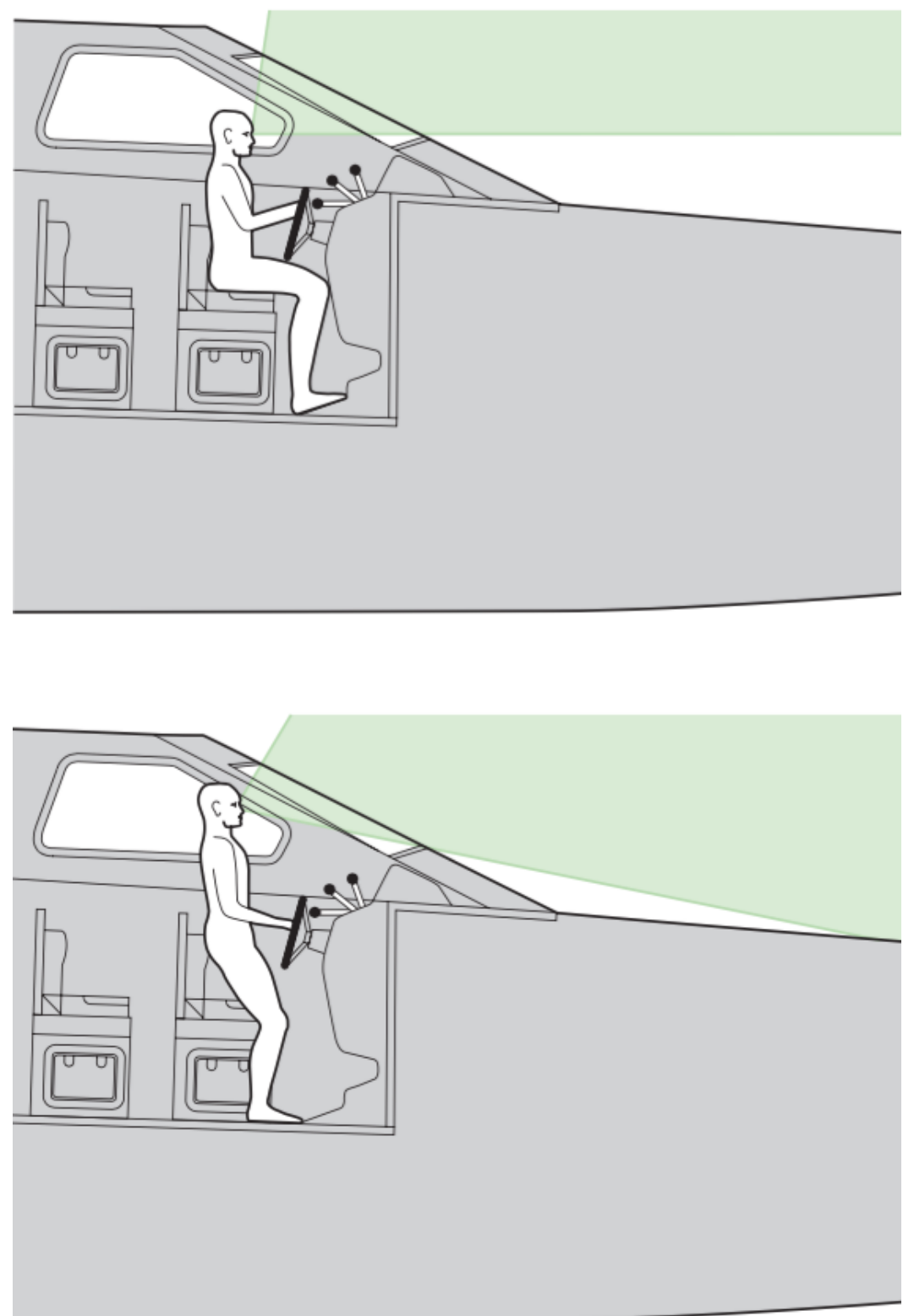

Figure 1.4: HSC operator in seated posture with restricted line of sight (top); and semi-squatted posture over the seat pan with increased line of sight (bottom) [2]. 


\subsubsection{Modelling Standards}

A number of standards and injury design rules have been widely accepted to assess environmental conditions involving whole-body vibration and impact. This section briefly assesses these standards for their applicability to the HSC environment.

\section{Vibration Dose Value and Ride Severity Index}

Vibration Dose Value (VDV) is a generic benchmark outlined in ISO 2631 [5] and is primarily used by the automobile industry to evaluate discomfort by computing rootmean-square (RMS) of an acceleration time history at the seat. A similar approach, known as the Ride Severity Index [6] uses statistical acceleration values from HSC transits to characterize seakeeping responses for potential structural damage and equipment malfunction. Average acceleration of the top $1 / 3 \mathrm{rd}, 1 / 10 \mathrm{th}$, and $1 / 100$ th peaks, as well as RMS acceleration are computed to assign a relative Ride Quality Index (RQI). The RQI can then be used to compare an operator's perception of ride quality between craft or sea conditions. Evaluating health and safety, however, is difficult since perceived discomfort in military personnel may not be aligned with that of the general civilian population. While useful for assessing structural integrity, this approach has limitations in applications to human injury in the HSC environment, and cannot be used to directly evaluate the effect of multi-DOF motions on occupants and craft design [7].

\section{Dynamic Response Index}

The Dynamic Response Index (DRI) [8] uses a single-degree-of-freedom (SDOF) lumped parameter model of the lumbar spine to assess single-event, seated vertical impacts such as jet aircraft seat ejections. The acceleration time history at the seat pan during the event is integrated twice to compute displacement. DRI values 
are then computed as non-dimensionalized displacements representing strain in the lumbar spine, ultimately derived as a function of maximum allowable compression of the occupant's spine. While this approach has been extended to multiple-impact events, it has limitations in applicability to stochastic high-acceleration environments with repetitive loading and cannot account for complex postures [7].

\section{ISO 2631 Part 5}

Part $5[9]$ is a recent addition to the ISO 2631 series standard for whole-body vibration containing repeated shock loading with particular consideration to lumbar spine dynamics. The concept is similar to DRI whereby a spinal response acceleration dose is computed using the number and magnitude of acceleration peaks considering deflections along all three axes. A linear, lumped-parameter model is used in the transverse directions, while a nonlinear neural network is used to evaluate response in the axial direction. Using induced acceleration, an equivalent static compressive stress dose $\left(\mathrm{S}_{\mathrm{ed}}\right)$ in the spine, normalized to an average eight-hour exposure, can be determined to estimate the likelihood of an adverse health effect occurring [9]. The methodology, however, is focused on long-term working conditions for the civilian population, producing erroneous results for high-magnitude impacts, thereby rendering its applicability to the HSC environment questionable [2,4].

\section{MADYMO}

MADYMO [10] provides finite-element and multi-body dynamic modelling capabilities using a variety of standard dummy and human models. The models are used extensively to predict injury parameters for vehicle collision testing; however, they seem to have limited transferability to other motion environments. When tested with experimental boat data, the models were insensitive to pitch motions resulting in significant limitations in the injury model 11 . 


\section{Summary}

Overall, there is a clear lack of a universally-accepted metric to characterize vibration exposure under the repetitive shock environment on HSC [4, 7]. A clear modelling framework is required that can be validated through experimental studies to characterize mechanical performance of the human body under such loading conditions. It is also important to note that human occupants can, and typically do, actively engage their muscles during HSC transits to mitigate shocks in an attempt to remain attached to the seat or deck and mitigate the effects of potentially-injurious shock loads. This effort is variable and difficult to capture, and is often missed in laboratory tests conducted with the use of passive masses in the form of crash test dummies or rigid mass stacks [4,12]. As a result, the contribution of active muscle engagement is often ignored in current modelling approaches and injury design rules.

\subsection{Literature Review}

In this literature review, generic dynamic modelling of the human body capabilities are first briefly investigated for their usefulness, followed by an exploration into the physiology of muscle contraction, mathematical modelling approaches for muscle contraction dynamics, and techniques for numerical solutions to develop a modelling framework for use with human movement.

\subsubsection{Human-body Dynamic Modelling}

To characterize postural stability movement in the sagittal and frontal planes, inverted pendulum rigid-body models are often employed 13 18. These consist of limb members that rotate about revolute joints and interact with their environments through external generalized forces or constrained geometry, as shown in Figure 1.5. 
The characteristics of joints specify constraints, thereby restricting DOF on attached limb members. Depending on the movement of interest, model biofidelity is improved through higher-DOF joints to account for out of plane motion [17, 19] and by the nature of internal and external forces through elaborate joint contact models and joint stiffness and damping characteristics to mimic soft tissue [19]. The corresponding equations of motion have been derived using Newtonian [13, 15 or Lagrangian dynamics [14] which represent mathematical models describing the relationship between generalized forces (kinetics) comprising ground reaction forces, muscular effort and gravitational forces; and the resulting rectilinear and angular positions, velocities, and accelerations (kinematics) of joints and limb segments. Depending on the quantities
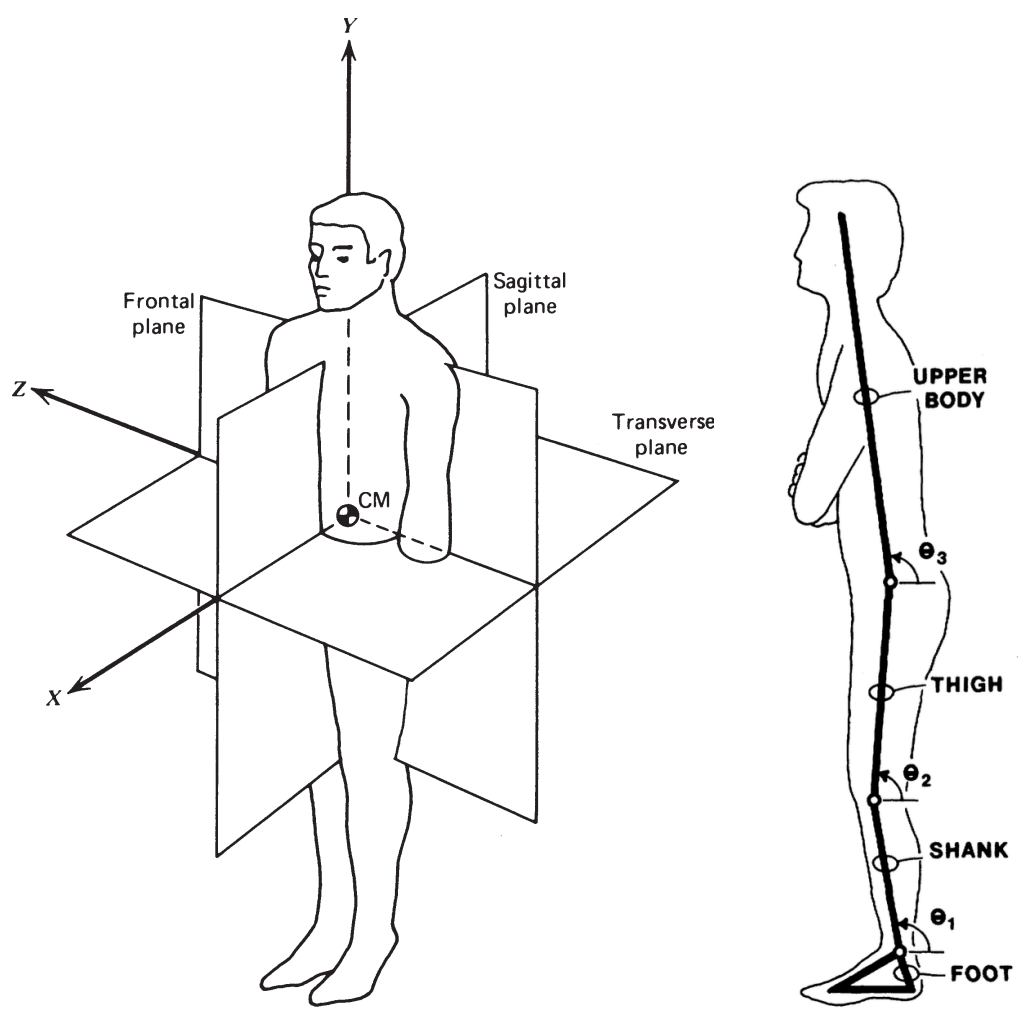

Figure 1.5: Spatial coordinate frame defining biomechanical movement 20, p. 47] (left); and classical four-mass sagittal-plane inverted pendulum model for postural stability analysis [15] (right).

of interest, the equations of motion describing musculoskeletal dynamics are evaluated 
using one of two approaches: forward and inverse dynamics. Forward dynamics is the technique associated with solving the equations as a system of ordinary differential equations (ODE) to compute kinematics of the human body from the known kinetic quantities of muscle forces and joint torques. While this is the preferred approach for predictive and controls applications in gait [19], postural stability [17, 18], and neuromuscular performance [21], it is computationally expensive and is often subject to drift due to the propagation of errors in simulation as small errors in joint torques can result in considerable errors in joint positions [22].

The computationally-simpler inverse dynamics approach has the advantage of inverting the equations of motion to solve differential algebraic equations (DAE) in order to compute the kinetics responsible after having observed the resulting kinematics. This is usually done through camera-based motion capture techniques [13, 16, 22, yielding positional trajectories of joints and limb segments. The displacements can be differentiated with respect to time to attain velocities and accelerations, which then can be used to compute the forces exerted. While computationally efficient in simulation, this approach lacks predictive capabilities and therefore is limited in cause and effect interpretations 23 . The majority of multi-body models in literature are actuated by joint torques, rather than muscle forces, which inherently results in a number of limitations. Torque generators represent the net effect of opposing muscle forces, and therefore lack insight on the physiological effects of muscle fatigue, energy expenditure, and time delays in response to external stimuli for force production [23]. By neglecting the effects of muscle redundancy, the effort involved in maintaining muscle activation for coordinated tasks [23] or stiffening of joints is significantly underestimated.

There are a number of reasons for this common simplification. Musculoskeletal dynamics are inherently under-determined [23], as the number of musculotendon units exceeds the number of DOF they actuate, such that there are an infinite number of 
combinations of muscle forces that can drive a specific joint with the same DOF. This becomes particularly challenging for inverse dynamic simulations, which require constrained optimizations $[19,22,24$ to achieve realistic muscle recruitment patterns. Despite tremendous efforts [25, p. 153], muscle forces still cannot be directly measured non-invasively. Surface electromyography (sEMG) can, however, act as a surrogate, with the use of mathematical models to estimate muscle activations for describing the dynamics of muscle contraction [23]. Furthermore, force production requires accurate mathematical representations of musculoskeletal geometry for the insertion and origin of musculotendon units to specify lengths and moment arms to compute resulting joint torque contributions [22]. While the elaborate geometry associated with the wrapping of muscle and tendons around bone and soft tissue has been generally characterized [26], these are very challenging to measure in vivo [22]. Despite these challenges, musculoskeletal dynamic models can offer improvements in the prediction of joint torques, muscle forces, metabolic energy consumption, and muscle recruitment patterns over traditional torque generator models [23].

\subsubsection{Muscle Anatomy and Physiology}

This section briefly describes the relevant anatomy and physiology for muscle contraction. There are three types of muscle [27:

1. Skeletal: which attach to bones via tendons and are responsible for voluntary movement;

2. Smooth: which are involuntary muscles which surround lumen (tubes) of the body such as blood vessels, and are responsible for controlling size of the lumen; and

3. Cardiac: which is a second involuntary muscle type that is sufficiently unique to be considered different, and makes up the majority of the heart's mass. 
Modelling techniques discussed in this thesis exclusively focus on skeletal muscle. Depending on its function, skeletal muscle is composed of a mix of three fibre types [27]:

1. Slow oxidative (Slow Twitch): these cells produce ATP (adenosine triphosphate - standard energy currency in the cell) via aerobic respiration, and are therefore critical for endurance activities;

2. Fast oxidative (Intermediate Twitch): these cells produce ATP aerobically, but can also switch over to glycolysis (anaerobic), and are thus used for activities requiring moderate strength at moderate durations; and

3. Glycolytic (Fast Twitch): these cells produce ATP anaerobically, and are thus used for high-strength, high-intensity activities.

Each muscle contains a mixture of these three types and recruitment of each fibre type is dependent on the task performed and forces required [27]. Henneman's size principle dictates that smaller loads requiring less force will recruit low-threshold slow-twitch fibres which are fatigue-resistant, whereas recruitment of high-threshold fast-twitch fibres which fatigue easily are reserved for larger forces [25, p. 6]. As shown in Figure 1.6, muscle tissue is composed of numerous bundles of muscle fibres known as fascicles. Each muscle fibre consists of parallel rods of myofibrils, which are responsible for causing muscle cell contraction. Myofibrils have a banded structure, with repeated series of sarcomere units, the basic contractile units of muscle. Each sarcomere unit has protein structures composed of thick myosin filaments surrounded by thinner actin filaments [27].

During muscle contraction, electrical impulses originating from the central nervous system (CNS) are sent, through motor neurons, to neuromuscular junctions which innervate a set of muscle fibres. The electrical impulses stimulate a flow of calcium ions into the sarcomeres, which causes the myosin filaments to slide against the actin 

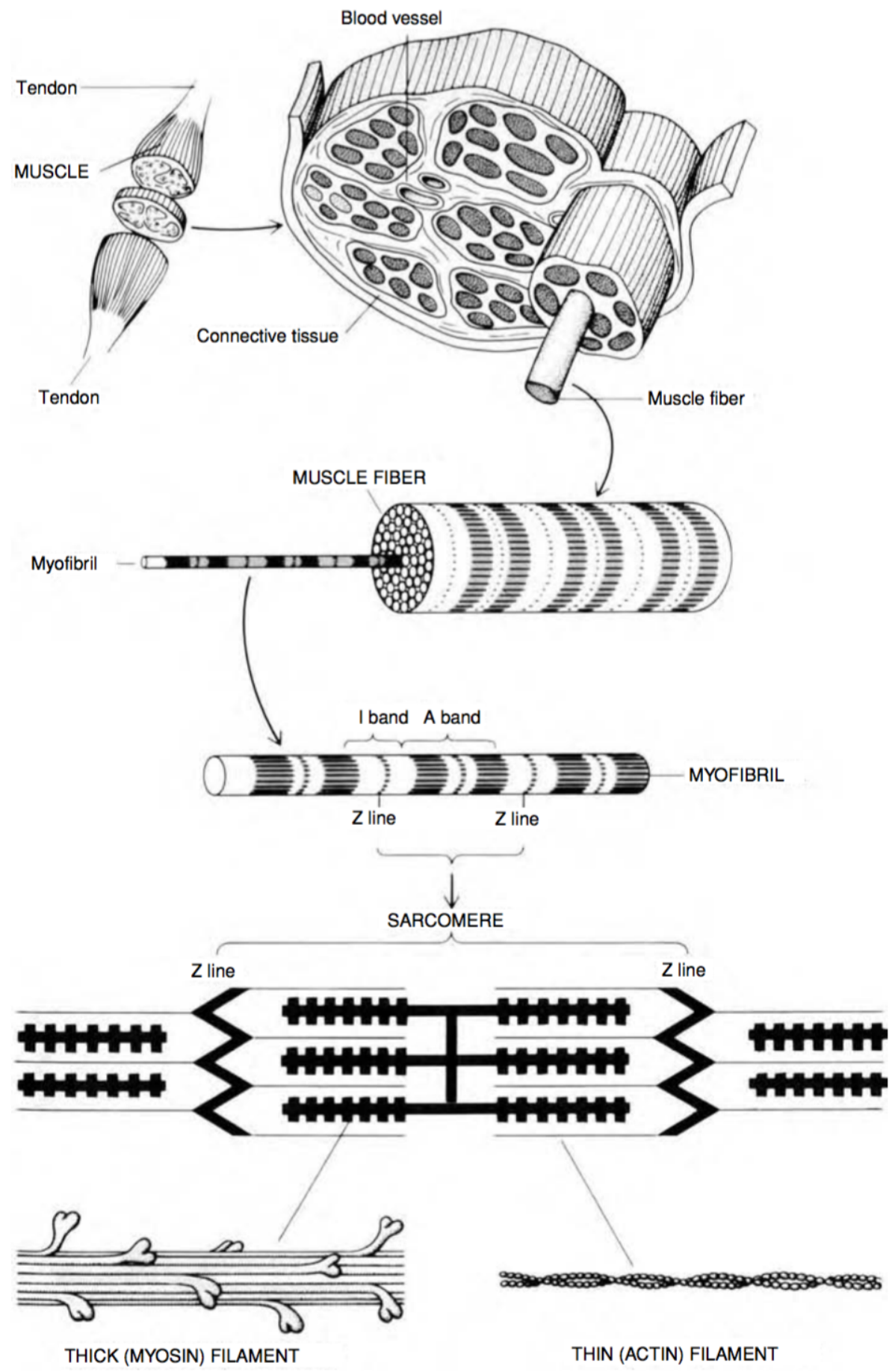

Figure 1.6: Organization of skeletal muscle structure illustrating the sliding filament model [28 facilitating muscle contraction [27]. 
filaments, thus shortening the sarcomere [24]. The sliding is a result of the myosin heads forming cross-bridges on neighbouring actin filaments in the presence of ATP, causing a ratcheting action which results in overlap of actin and myosin filaments. The overlap causes the muscle to contract in length, responsible for the dark bands seen in the sarcomeres, and the amount of overlap is directly proportional to the force generated 28,29$]$.

\subsubsection{Muscle Models}

Muscle mechanics are an integral combination of mechanical, electrical, and chemical dynamic systems working simultaneously [23]. A good mathematical model must integrate the relationship between these systems to account for the neural and mechanical activity in force generation to simulate human movement. Experimentation alone is not sufficient to accurately model force production [25, p. 153] for a number of reasons. EMG measurements do not contain information on joint configuration, which reflect muscle and tendon stiffness and their resulting forces [22, 23]. Additionally, EMG measurements are representative of the neural excitation provided to motor neurons, and thus require treatment of the time delays associated with force production. Therefore, the dynamics involved in these processes require coupling with skeletal and musculotendon kinematics, as well as the dynamics involved to estimate muscle activations for force production.

The following sections investigate the biophysical and phenomenological techniques used in literature to model muscle contraction and force generation, and evaluate their effectiveness with regards to simulation and capabilities for postural stability studies. 


\section{Activation Dynamics}

Neural excitation refers to the electrical potential that spreads across the muscle as it is innervated, and can be estimated by measured sEMG [22]. The time delay associated with force generation in the muscle fibres is generally modelled by a firstorder differential equation of the form [24:

$$
\frac{\mathrm{d} a}{\mathrm{~d} t}=(u-a)\left(\frac{u}{\tau_{\text {act }}}+\frac{1-u}{\tau_{\text {deact }}}\right)
$$

where $u$ represents neural excitation, often derived from sEMG, $a$ is muscle activation, and $\tau_{\text {act }}$ and $\tau_{\text {deact }}$ are the activation and deactivation time constants for a given muscle.

\section{Huxley-type Models}

Huxley [28, 29] developed the sliding filament model that describes force production at the microstructure level due to cross-bridge formation between actin and myosin filaments as shown in Figure 1.6. The force produced $F(t)$ is proportional to the number of attached cross-bridges with distribution function $n(x, t)$, displacement $x$ at time $t$, and contraction velocity $v(t)$; as well as the respective attachment and

detachment rates $f(x)$ and $g(x)$. The corresponding relationships are given by [24,30]:

$$
\begin{gathered}
\frac{\mathrm{d} n}{\mathrm{~d} t}=\frac{\partial n}{\partial t}-v(t) \frac{\partial n}{\partial x}=(1-n) f(x)-n g(x) \\
F(t)=\frac{\eta \kappa \Gamma s(t)}{2 \lambda} \int_{-\infty}^{\infty} x n(x, t) \mathrm{d} x
\end{gathered}
$$

where $\eta$ represents the number of cross-bridges per unit volume with physiological cross-sectional area (PCSA) $\Gamma$ of the muscle, sarcomere length $s(t)$, the distance 
between successive binding sites $\lambda$, and spring with constant $\kappa$ representing the crosslink between the myosin head and actin filament.

While this approach models the mechanics of cross-bridge formation and the resulting force-displacement relationships accurately, scaling these relationships to whole muscles has been shown to be problematic in modelling velocity dependence, particularly for use with non-isometric contractions [23].

Attempts at experimental validation such as Zahalak's [30] work, have provided improvements to Huxley's model in simulating eccentric contractions at the cost of high complexity requiring numerous empirical parameters that cannot be physically explained. Consequently, this type of framework is not feasible for use with human simulations of movement involving multiple muscles 22, 23].

\section{Hill-type Models}

Hill [31, 32] was interested in modelling the external behaviour of muscle rather than its underlying physiology [22]. In doing so, a lumped parameter approach was taken, as shown in Figure 1.7, whereby muscle is modelled as a damped (B), active forcegenerating contractile element $(\mathrm{CE})$ with a parallel elastic $(\mathrm{PE})$ element representing its passive elasticity, attached to a series elastic (SE) element to represent the tendon. The force-generating capacity of muscle is dependent on length and velocity such that actin and myosin filaments have maximum overlap at an optimal length, producing the greatest capacity to generate force. Force drops as length deviates from this optimal position in either direction. Force is also scaled by the muscle activation signal, which allows a maximally-innervated musculotendon unit to produce a maximum isometric force at its optimal fibre length, a quantity unique to each musculotendon unit. These relationships, along with stiffness and damping parameters of muscle and tendon, are empirically modelled from individual muscle fibres, including the nonlinear elastic properties of muscle and tendon tissue 22, 24. 


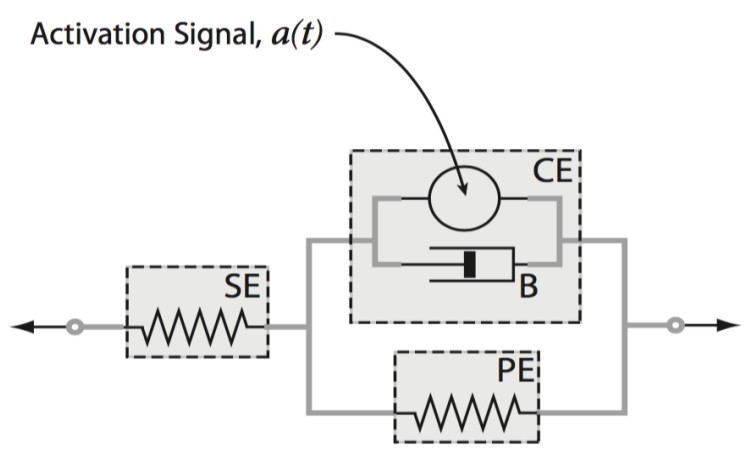

Figure 1.7: Hill's lumped parameter model describing the dynamics of muscle contraction [24].

Zajac [33] refined Hill's model by non-dimensionalizing these relationships such that they could be scaled by its optimal fibre length, $L_{o}^{M}$, maximum isometric force $F_{i s o}^{\max }$, and tendon slack length $L_{s}^{T}$ to represent any skeletal musculotendon unit [24]. In doing so, the lumped parameter model can be represented as a 1DOF system, modelled as a first-order differential equation for each musculotendon unit. The muscle force can then be computed by $[22]$ :

$$
F^{M}=F_{i s o}^{\max }\left(a f_{L} f_{V}+\bar{F}^{P E}\right)
$$

where $f_{L}$ and $f_{V}$ represent the force-length and force-velocity relationships of the muscle's contractile element, and $\bar{F}^{P E}$ represents the passive elastic force of muscle, each expressed using non-dimensional quantities. This type of framework allows scalability to large-scale human movement such that multi-body dynamic models can be actuated by numerous musculotendon units in simulation with computational feasibility. 


\subsubsection{Simulation}

Given that muscles are only capable of generating contractile forces, multi-body models consist of numerous muscles arranged about each joint, both for stability, and such that muscles work as agonist-antagonist pairs in opposition to produce controlled and coordinated motion. This leaves the system inherently underdetermined. In addition, numerous muscles such as the gastrocnemii (knee flexors/ankle plantarflexors), biceps femoris (hip extensor/knee flexor), and rectus femoris (hip flexor/knee extensor) are biarticular as shown in Figure 1.8, in that they produce torques about multiple joints. These muscles have a large impact on energy expenditure during coordinated motion, where energy can be transferred between joints by performing positive work on one joint and negative work on another, which reduces the net change in muscle length as compared to two individual muscles performing the same task [20, p. 102-104]. Reproducing these complex recruitment patterns in simulation is done through optimization to solve the muscle redundancy problem. Biofidelic reproductions of recruitment pat-
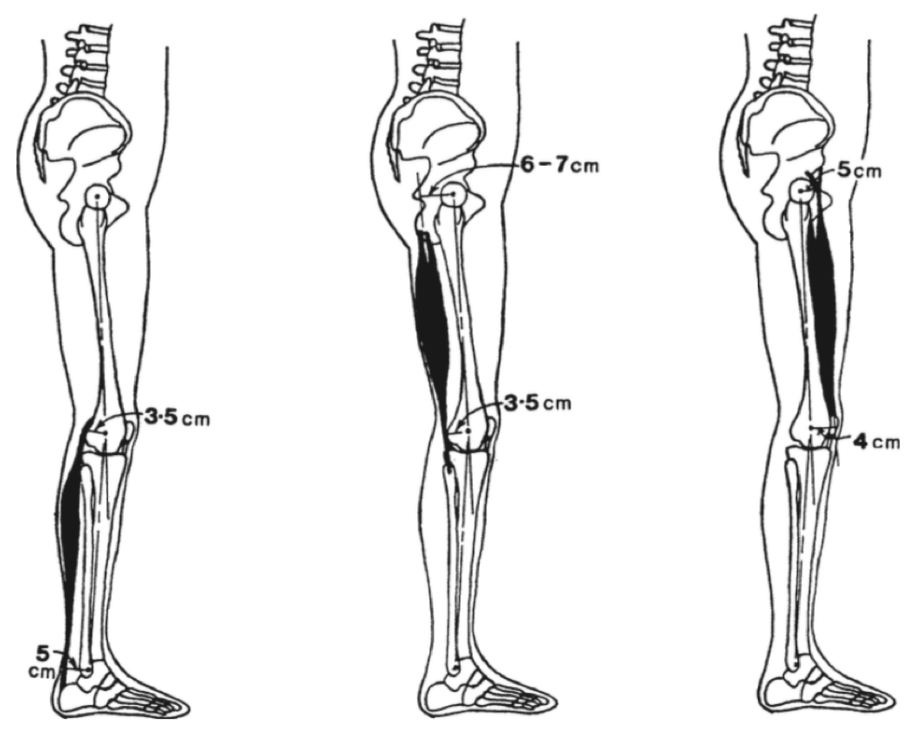

Figure 1.8: Biarticular muscles (left to right): gastrocnemii, biceps femoris, and rectus femoris shown with the respective joint's moment arms [20, p. 104].

terns and force generation typically make use of an objective cost function of the 
following form 23,24 :

$$
J=\sum_{i=1}^{n}\left(\frac{F_{i}}{N_{i}}\right)^{p}
$$

where the quotient of muscle force $F$ and a physiological muscle parameter $N$ is minimized for $n$ muscles involved in the movement. A common practice of using PCSA for $N$ with an exponent of $p=3$ has been shown to produce recruitment patterns that are consistent with the minimization of muscle fatigue 34, 35]. There are two commonly-used approaches for muscle redundancy: static and dynamic optimization.

Static optimization refers to the process of minimizing Equation 1.5 at each instant of time to minimize muscle forces. This removes any dynamic dependency in muscle forces across the duration of the task and does not require time integration, thereby making it computationally simple [24]. This is often the desired approach to solve the inverse dynamics problem to attain muscle forces when muscle activations are not available.

In contrast, dynamic optimization requires coupling of activation, muscle contraction, and skeletal dynamics, taking neural excitation as input to compute the resulting movement while simultaneously computing the optimal excitation signals to satisfy desired performance criteria [23, 24]. In doing so, time histories of the complete movement are considered, which, while computationally expensive, is useful for optimization of physiological performance parameters such as minimum energy expenditure in gait [19] and maximum vertical jump height [36], making dynamic optimization ideal for both forward and inverse dynamic simulations. Anderson and Pandy [35] showed that static and dynamic optimizations can produce similar results in predicting muscle forces and joint contact forces during some movements such as gait, due to the similarity in minimizing muscle fatigue at each instant of time as well as across the gait cycle 24$]$. 


\subsubsection{Summary}

Muscle models have the potential to aid in pathological studies examining changes in movement and muscle dynamics during the onset of fatigue and injury, as well as evaluate the effectiveness of rehabilitation. By modelling the effects of muscle engagement, the influence of human-machine interaction can be studied for assistive devices such as suspension seating on HSC as well as the effects of force input from seats in the semi-squatted position adopted by HSC occupants. These dynamic simulations can reveal control strategies to cope with, and reduce the incidence of injury and fatigue. Finally, with an appropriate construct of forward- or inverse-dynamics, quantities of interest can be computed efficiently through the collection of experimental data, or for predictive purposes of kinetics, kinematics, and work involved with human movement.

\subsection{Motivation and Scope of Research}

To date, the muscle modelling techniques described here have been primarily applied to gait and neuromuscular jump performance. This study seeks to develop a dynamic modelling framework for application in postural stability studies to provide predictive capabilities for musculotendon forces and joint torques. This is intended as a tool to study muscle recruitment patterns and energy expenditure for maintaining postural stability in motion environments. Of particular interest is the use of this framework to address the muscle involvement in maintaining postural stability in the harsh environments onboard HSC, as well as the high metabolic cost in doing so, as a design rule for reduction of injury and safe operational planning. 


\subsection{Thesis Overview}

In this thesis, model development of a musculoskeletal model and energy expenditure model is discussed in Chapter 2 to derive the resulting equations of motion for use with experimental validation. The framework consists of a 3DOF inverted pendulum skeletal dynamic model actuated by the seven major musculotendon units of the lower extremity. Chapter 3 details experimental data collection, instrumentation and post-processing techniques to obtain the input variables to the model as well as parameters for validation. In Chapter 4 , the calibration procedures for the model are discussed followed by a presentation of validation data to assess the predictive capability between model output and acquired data for joint torques and metabolic energy. Lastly, Chapter 5 presents a summary of the work along with future considerations for improvements in the modelling framework presented here. 


\section{Chapter 2}

\section{Model Development}

\subsection{Overview}

When the body attempts to perform a motor task, neural excitation signals are generated by the CNS to activate muscles, subsequently generating forces that are transmitted, through tendons, to the skeletal system to execute the task 33].

This chapter is intended to detail the modelling process for the neuromusculoskeletal dynamics of human movement in a motion environment. The objective of the model is to predict musculotendon forces and joint torques from neuromuscular signals under prescribed motion, as well as to simultaneously compute energy expended by the muscles. The modelling process involves four primary steps [22] which are illustrated in Figure 2.1.

1. Muscle Activation Dynamics: to compute muscle activation $a(t)$ from neural excitation $u(t)$, which is derived from surface electromyography $e(t)$;

2. Musculotendon Contraction Dynamics: to compute muscle forces $F(t)$ from muscle activations;

3. Musculoskeletal Geometry: to determine muscle moment arms $r^{M}\left(\theta_{j}(t)\right)$ as a function of joint configuration to compute joint torques $T(t)$ from muscle 


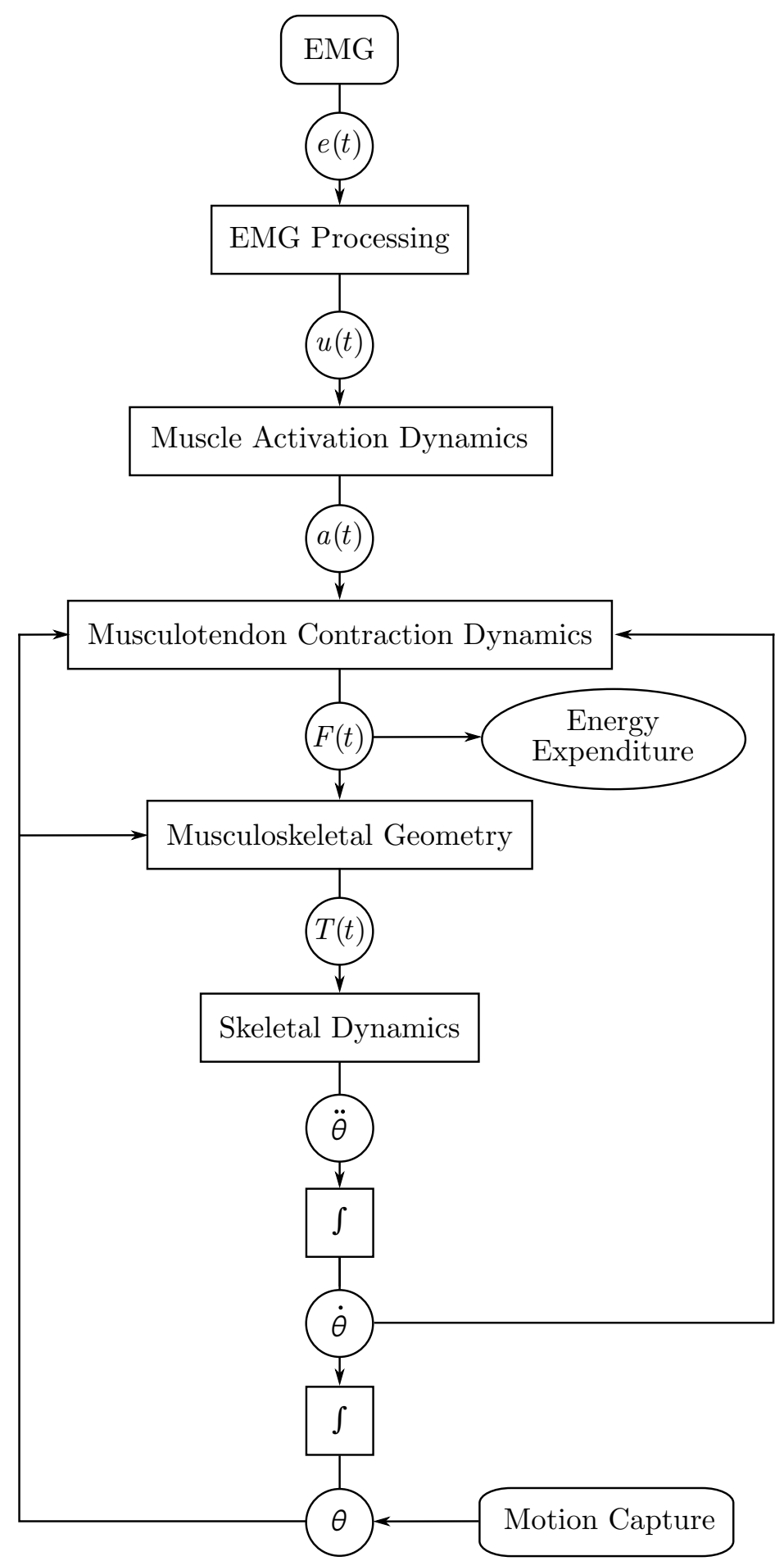

Figure 2.1: Neuromusculoskeletal modelling process. 
forces; and

4. Skeletal Dynamic Equations of Motion: to determine joint kinematics $\theta(t)$, $\dot{\theta}(t), \ddot{\theta}(t)$ from joint torques using the rigid-body dynamic equations of motion of the various body segments as they rotate about their respective joints.

This chapter details the modelling process with a description of the model and underlying assumptions in Section 2.1.1, the four primary steps of neuromusculoskeletal dynamic modelling in Sections 2.2, 2.3, 2.5, and 2.6, and development of a muscle expenditure model in Section 2.4.

\subsubsection{Model Description}

Forward dynamics uses neural excitation derived from EMG as an input to determine muscle activations to estimate musculotendon forces and torques, while inverse dynamics approaches the problem from the other end where joint kinematics are measured along with external forces acting on the body in order to determine joint torques. A forward-dynamic computation of human body kinetics has the benefit of providing a better estimate of the simultaneous muscle involvement to exert net torques to actuate a joint. Particularly in applications requiring controlled motions (e.g., slow extension of the arm with a sizeable load), these muscle torques act in opposition, to stiffen a joint, or control the speed of motion. This results in muscle redundancy which may produce large muscle forces but results in a small net torque applied about the joint. In this case, an inverse dynamics approach would yield small net joint torques due to the cancelling effect of opposing muscle force components, and thus fail to estimate muscle forces which could have large power requirements to execute the task.

This is often the case in maintaining postural stability where an individual, under large accelerations in a motion environment, may stiffen joints through muscle 
contractions to stabilize the body. In this study, an EMG-based forward dynamics modelling approach is selected to estimate the kinetics and energetics in maintaining postural stability on planar motion environments.

The intended model is developed under the following underlying assumptions.

1. As shown in Figure 2.6, the body is modelled as a three-segment inverted pendulum with shanks, thighs, and combined head, arms, and trunk (HAT) - a common approach $[14,21,38$. Hence, the model is assumed to be bilaterally symmetric, whereby both shanks and both thighs move in unison.

2. The above limb segments are actuated about the ankle, knee, and hip joints, which are assumed to be connected to the limb as single-degree-of-freedom (SDOF) revolute joints with fixed joint centres such that the resulting motion is restricted to the sagittal plane.

3. The feet are assumed to be rigidly attached to the supporting deck e.g. boat deck without sliding or pivoting, and their motion is completely described by surface translations and rotations of the boat deck.

4. The prescribed boat motion is restricted to 3DOF with deck translations in surge and heave, $\dot{x}$ and $\dot{y}$ respectively, as well as rotation in pitch, $\dot{\theta}_{S}$, all of which are completely known from measured data; see Figure 2.6.

5. While there are over 40 muscles in the lower extremity [39], the seven largest force-producing muscle groups are modelled in this study, as shown in Figure 2.2 , comprising the: glutei (GLU), rectus femoris (RF), vasti (VAS), biceps femoris $(\mathrm{BF})$, gastrocnemius (GAS), tibialis anterior (TA), and soleus (SOL). 


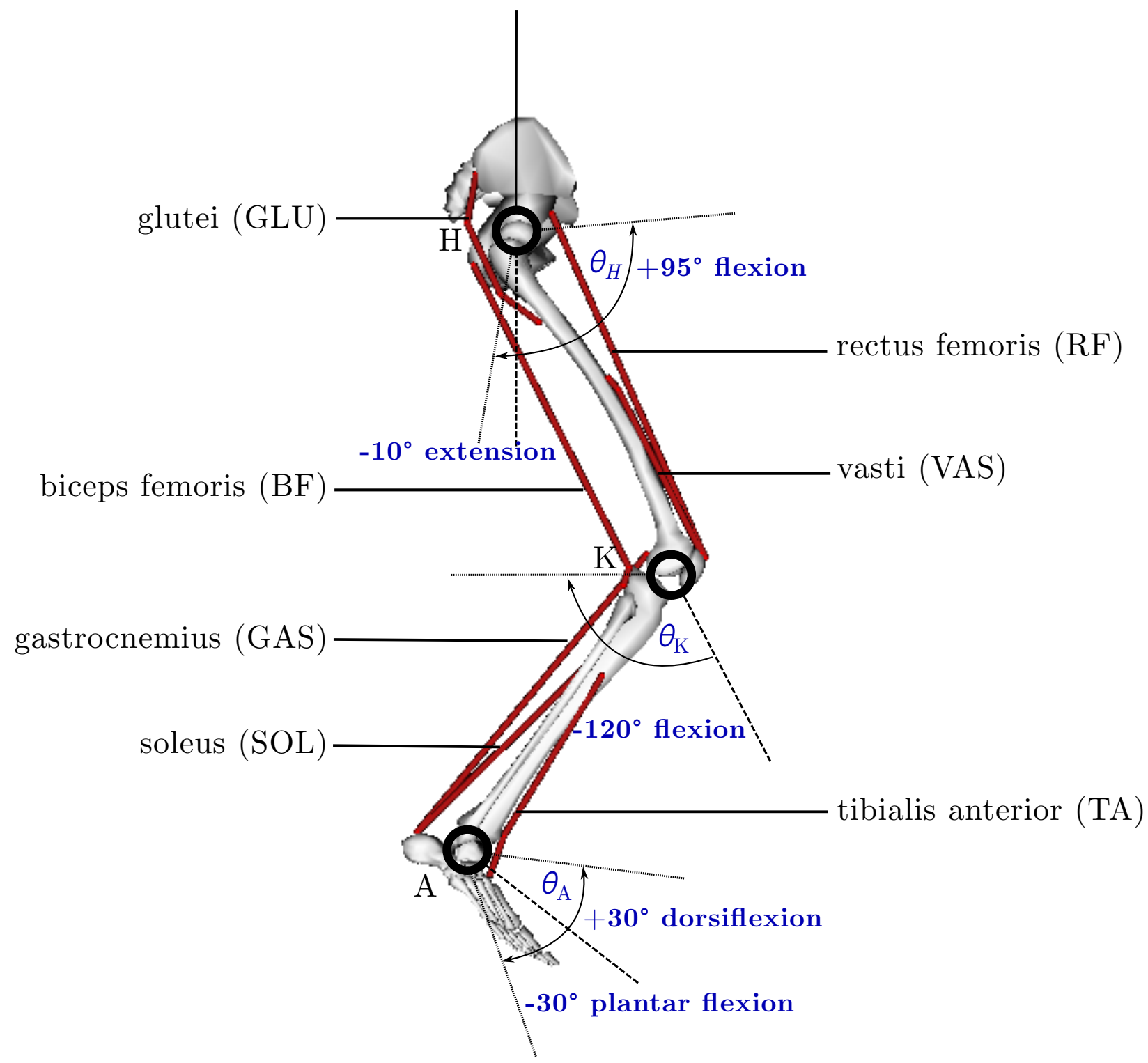

Figure 2.2: Four-segment musculoskeletal model of foot, leg, thigh, and HAT actuated by seven major force-producing muscles about ankle, knee, and hip, with accurate depiction of muscle origin and insertion sites on bone [37]. 


\subsection{Muscle Activation Dynamics}

EMG is used to determine a muscle's activation profile. A raw EMG signal, through rectification, systematic filtering, and normalization (detailed in Chapter 3), produces an EMG envelope signal $e(t)$ varying between 0 and 1 . An activation dynamics model estimates muscle activation $a(t)$ from $e(t)$, which is in turn used to determine muscle forces $[22]$.

\subsubsection{Twitch Response}

When a muscle fibre is excited by an action potential, it generates a twitch response which can be represented by a damped linear second-order differential equation [22]:

$$
u(t)=M \frac{\mathrm{d}^{2} e(t)}{\mathrm{dt}^{2}}+B \frac{\mathrm{d} e(t)}{\mathrm{dt}}+K e(t)
$$

where $M, B$, and $K$ represent inertial, damping, and stiffness parameters of the response. This type of response has been used to determine neural excitation $u(t)$ from the measured EMG envelope $e(t)$. The $e(t)$ signal is derived by filtering, rectifying, and low-pass filtering the EMG signal for its envelope while $u(t)$ is derived via subsequent post-processing of the EMG envelope as detailed in Section 3.5.1. Discretizing this response for use with measured data points yields [22]:

$$
u_{j}(t)=\alpha_{t} e_{j}(t-\delta)-\beta_{1} u_{j}(t-1)-\beta_{2} u_{j}(t-2)
$$


where:

$$
\begin{aligned}
\beta_{1} & =\gamma_{1}+\gamma_{2} \\
\beta_{2} & =\gamma_{1} \gamma_{2} \\
\left|\gamma_{1}\right| & <1 \\
\left|\gamma_{2}\right| & <1 \\
\alpha_{t}-\beta_{1}-\beta_{2} & =1
\end{aligned}
$$

It should be noted that for a given muscle $j, \alpha_{t}$ is the gain coefficient, $\beta_{1}$ and $\beta_{2}$ are recursive coefficients, and $\delta$ is the electromechanical delay. Parameters $\gamma_{1}$ and $\gamma_{2}$ dictate the impulse response of the second-order filter - if they are both positive, the response is under-damped, if the values are instead negative, or of different signs with $\left|\gamma_{1}\right|>\left|\gamma_{2}\right|$, the response is over-damped. The damped second-order response stretches the duration of the processed EMG while the electromechanical delay improves the synchronization between activation and force production [22].

If $\gamma_{1}$ and $\gamma_{2}$ are known, $\beta_{1}, \beta_{2}$, and $\alpha$ can be found from the above equations. An electromechanical delay, $\delta$, is included in the discretized recursive filter which accounts for the delay between the neural signal and the start of the resulting twitch force. This delay has been reported to range between 10 and 100 ms, with a typical value of $40 \mathrm{~ms}, 22,40$.

\subsubsection{Activation Model}

Muscles cannot generate force instantaneously and experience a systematic time delay between receiving neural excitation signals and the resulting force generated. To account for this time delay, a first-order nonlinear differential equation is used to represent the muscle's activation state, $a(t)$, as a function of the neural excitation, 
$u(t)$, it receives 41,42 :

$$
\frac{\mathrm{d} a}{\mathrm{~d} t}=\frac{u-a}{\tau_{a}(a, u)}
$$

where $\tau_{a}(a, u)$ is a time constant that varies with the level of muscle activation as well as the rate of change of activation:

$$
\tau_{a}(a, u)= \begin{cases}\tau_{a c t}(0.5+1.5 a) & u>a \\ \tau_{\text {deact }} /(0.5+1.5 a) & u \leq a\end{cases}
$$

Here, $\tau_{\text {act }}$ and $\tau_{\text {deact }}$ are the activation and deactivation time constants, with a value of $10 \mathrm{~ms}$ and $40 \mathrm{~ms}$ respectively, and can be modified to account for age-related changes in muscle mechanics 41.

An alternative, simpler formulation developed by Lloyd [40,43] has been shown to yield adequate solutions without requiring integration:

$$
a(t)=\frac{e^{G u(t)}-1}{e^{G}-1}
$$

In this case, a single parameter $G$, between -3 and 0 , is used to represent the nonlinearity and can be tuned in the calibration process, whereby $G=-3$ produces a highly nonlinear relationship, while $G=0$ produces a linear relationship [22]. For simplicity, Lloyd's model is used in this study.

\subsection{Musculotendon Contraction Dynamics}

Contraction dynamics is the mathematical model transforming muscle activation to musculotendon force. The common approach adopted for multi-body systems is the 
use of Hill-type models which express muscle dynamics in the form of a first-order differential equation for each muscle. As shown in Figure 2.3, a musculotendon actuator
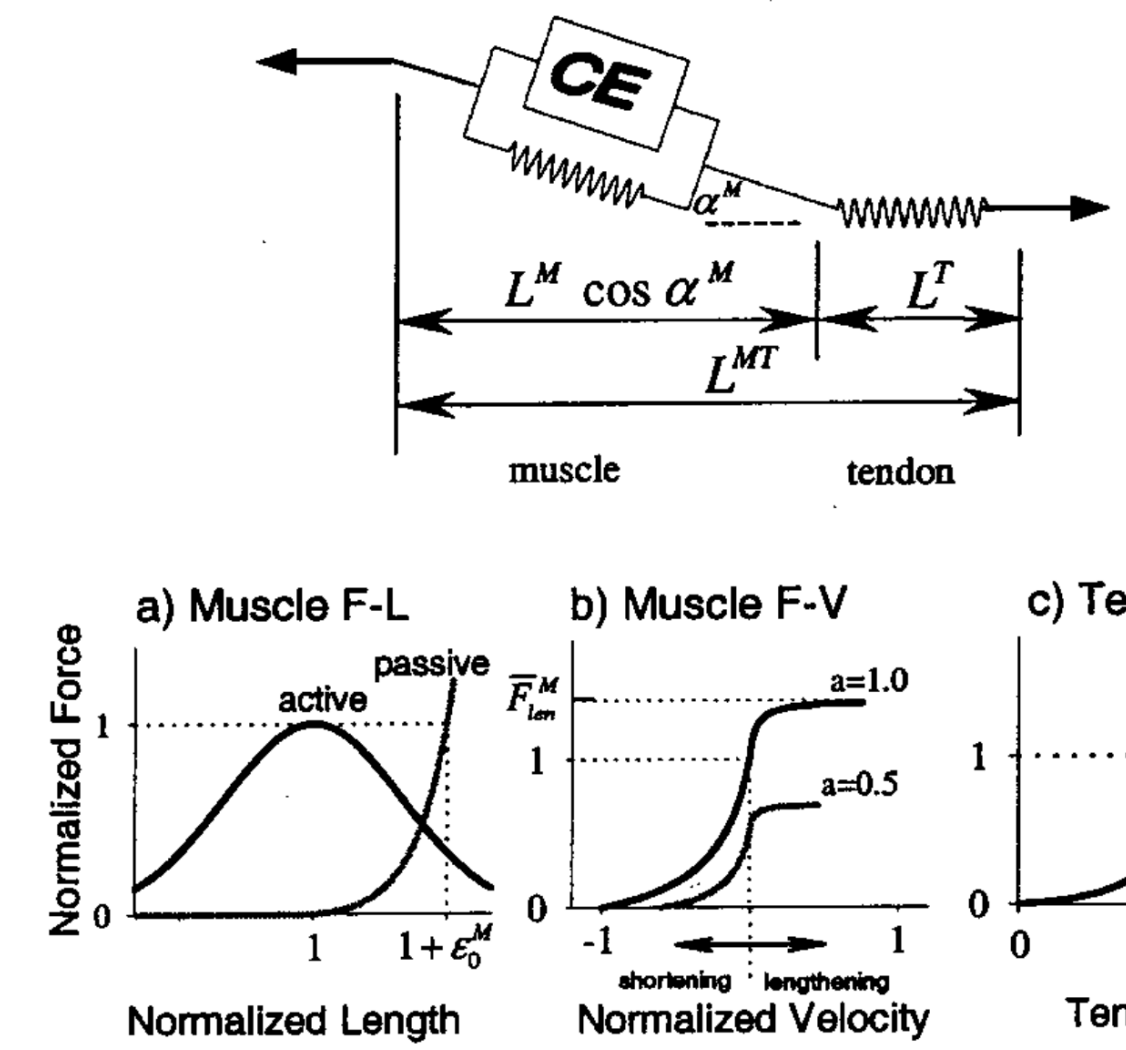

c) Tendon

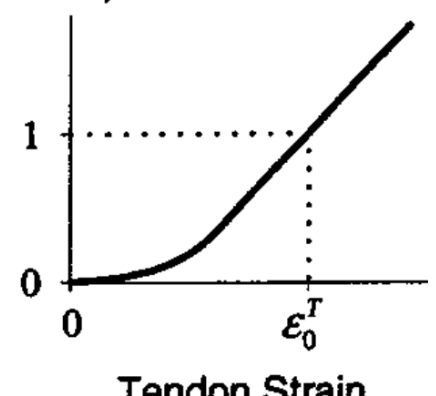

Figure 2.3: Hill-type musculotendon model describing contraction dynamics. (a) Active and passive force-length relationships of muscle; (b) Active forcevelocity relationship of muscle; (c) Tendon force-strain relationship [41].

consists of an active contractile element (force generator), a passive, parallel elastic element to model muscle stiffness, and a series elastic element to model the tendon. The contractile element develops tension when the muscle tissue is excited by the central nervous system. The force it generates $F^{M}$ varies nonlinearly with the length of muscle tissue $L^{M}$, which is represented by active force-length curve $f_{L}$. This curve peaks at the optimum muscle fibre length $L_{o}^{M}$, producing the maximum isometric force the muscle can generate $F_{i s o}^{\max }[41,42$. By using the normalized force-length and force-velocity relationships shown here, any musculotendon unit can be modelled 
as a function of its maximum isometric force $F_{i s o}^{\max }$, optimal muscle fibre length $L_{o}^{M}$, and maximum contraction velocity $V_{\max }^{M}$.

Muscle contractions are generally categorized as isometric (constant length), concentric (shortening), or eccentric (lengthening). During concentric contractions, the muscle produces force in the same direction as the fibre velocity $V^{M}$, whereas force and velocity are in opposite directions during eccentric contractions such that the muscle acts to stabilize the load. During non-isometric contractions, muscle force also varies nonlinearly with contraction velocity, which is denoted as the active forcevelocity relationship $f_{V}$ 41, 42].

As the muscle tissue stretches, its passive stiffness produces a force proportional to its length. This relationship is denoted by the passive force-length relationship $F^{P E}$, modelled as a nonlinear spring $[41,42]$.

By combining these three relationships, muscle force $F^{M}$ can be computed as:

$$
F^{M}=F_{i s o}^{\max }\left[a f_{L}\left(\bar{L}^{M}\right) f_{V}\left(\bar{V}^{M}\right)+\bar{F}^{P E}\left(\bar{L}^{M}\right)\right]
$$

In doing so, each force relationship is normalized to the given muscle's maximum isometric force $F_{i s o}^{\max }$, optimal muscle fibre length $L_{o}^{M}$, and maximum contraction velocity $V_{\max }^{M}$, typically set to $10 L_{o}^{M} \mathrm{~s}^{-1}$ for young adults [41], such that:

$$
\begin{gathered}
\bar{L}^{M}=L^{M} / L_{o}^{M} \\
\bar{V}^{M}=V^{M} / V_{\max }^{M} \\
=V^{M} /\left(10 L_{o}^{M}\right)
\end{gathered}
$$

Tendons are passive tissue and can produce considerable forces when stretched from their slack length $L_{s}^{T}$, allowing them to be modelled as nonlinear springs denoted by 
the tendon force-length curve $F^{T}$. As shown in Figure 2.3, muscle fibre is attached to tendon at a pennation angle $\alpha^{M}$. By neglecting the mass of the muscle, the equilibrium equation between tendon and muscle forces can be expressed as:

$$
\begin{aligned}
F^{M} \cos \alpha^{M}-F^{T} & =0 \\
F_{i s o}^{\max }\left[a f_{L}\left(\bar{L}^{M}\right) f_{V}\left(\bar{V}^{M}\right)+\bar{F}^{P E}\left(\bar{L}^{M}\right)\right] \cos \alpha^{M}-F_{i s o}^{\max } \bar{F}_{T}\left(\bar{L}^{T}\right) & =0
\end{aligned}
$$

whereby Equation 2.10 is obtained by substituting Equation 2.6 into Equation 2.9. Tendon length is also normalized to optimal muscle fibre length:

$$
\bar{L}^{T}=L^{T} / L_{o}^{M}
$$

By normalizing the length and velocity of muscle and tendon, forces for any musculotendon actuator can be computed using four muscle-specific parameters:

$$
\begin{aligned}
L_{o}^{M} & \text { : optimal muscle fibre length; } \\
L_{s}^{T} & \text { : tendon slack length; } \\
F_{i s o}^{\text {max }} & \text { : maximum isometric force (at optimal muscle fibre length); and } \\
\alpha_{o}^{M} & : \text { muscle pennation angle at optimal muscle fibre length. }
\end{aligned}
$$

For the seven musculotendon actuators considered in this study, these parameters have been experimentally determined for the average individual [37], and tabulated in Table 2.1 along with their respective proportions of fast-twitch fibres $\mu_{F T}$. The force-length and force--velocity relationships for tendon and muscle described here are shown in Figure 2.3, and their mathematical models are derived in Sections 2.3.12.3 .5 . 
Table 2.1: Musculotendon parameters for seven major muscles of the lower extremity. Fibre composition taken from Umberger [44], all other values taken from Delp 37].

\begin{tabular}{lcccccc}
\hline Muscle & Abbrev. & $L_{o}^{M}(\mathrm{~m})$ & $L_{s}^{T}(\mathrm{~m})$ & $F_{i s o}^{\max }(\mathrm{N})$ & $\alpha_{o}^{M}(\mathrm{rad})$ & $\mu_{F T}$ \\
\hline Glutei & GLU & 0.1629 & 0.1407 & 1944 & 0 & 0.45 \\
Rectus Femoris & RF & 0.1329 & 0.3613 & 1169 & 0.0872 & 0.65 \\
Vasti & VAS & 0.2162 & 0.1368 & 4530 & 0.0524 & 0.50 \\
Biceps Femoris & BF & 0.1282 & 0.3835 & 2594 & 0 & 0.35 \\
Tibialis Anterior & TA & 0.1101 & 0.2504 & 1759 & 0.0873 & 0.25 \\
Gastrocnemii & GAS & 0.1031 & 0.4125 & 2241 & 0.2967 & 0.50 \\
Soleus & SOL & 0.0297 & 0.4125 & 5137 & 0.4363 & 0.20 \\
\hline
\end{tabular}

\subsubsection{Muscle Passive Force-length Relationship}

Passive muscle force is generated due to the elasticity of muscle tissue that is in parallel with the contractile element in Figure 2.3. These forces are very small for muscle fibre lengths below $L_{o}^{M}$, and increase nonlinearly thereafter. In doing so, the normalized force of the passive element can be expressed as [41]:

$$
F^{P E}=F_{i s o}^{\max } \bar{F}^{P E}
$$

where

$$
\bar{F}^{P E}\left(\bar{L}^{M}\right)= \begin{cases}1+\frac{k^{P E}}{\varepsilon_{0}^{M}}\left(\bar{L}^{M}-\left(1+\varepsilon_{0}^{M}\right)\right) & \bar{L}^{M}>1+\varepsilon_{0}^{M} \\ \frac{e^{k^{P E}}\left(\bar{L}^{M}-1\right) / \varepsilon_{0}^{M}}{e^{k^{P E}}} & \bar{L}^{M} \leq 1+\varepsilon_{0}^{M}\end{cases}
$$

Parameter $k^{P E}$ is an exponential shape factor set to 4 [37], and $\varepsilon_{0}^{M}$ is the passive muscle strain due to maximum isometric force set to 0.6 for young healthy adults [37. 


\subsubsection{Muscle Active Force-length Relationship}

The active force-length relationship represents the force-generator capability of the muscle's contractile element, which can be approximated as a Gaussian function that peaks at $L_{o}^{M}$. This curve scales linearly with muscle activation and can thus be approximated by the following 41$]$ :

$$
F_{a}^{M}=a f_{L} F_{i s o}^{\max }
$$

where

$$
f_{L}=e^{-\left(\bar{L}^{M}-1\right)^{2} / \gamma_{L}}
$$

and $\gamma_{L}$ is a shape factor set to 0.6 [37] to approximate the force-length relationship of sarcomeres [41].

\subsubsection{Muscle Force-velocity Relationship}

In order to simulate the model using integration, the force-velocity $f_{V}$ relationship is usually inverted to express fibre velocity $V^{M}$ as a function of force and the forcelength relationship [41]:

$$
\dot{L}^{M}=V^{M}=(0.25+0.75 a) V_{\max }^{M} \frac{\bar{F}^{M}-a f_{L}}{b}
$$

where $V_{\text {max }}^{M}$ is the maximum contraction velocity and $b$ is a parameter computed based on whether the fibre is shortening $\left(\bar{F}^{M} \leq a f_{L}\right)$ or lengthening $\left(\bar{F}^{M}>a f_{L}\right)$ :

$$
b= \begin{cases}a f_{L}+\bar{F}^{M} / S_{f} & \bar{F}^{M} \leq a f_{L} \\ \frac{\left(2+2 / S_{f}\right)\left(a f_{L} \bar{F}_{l e n}^{M}-\bar{F}^{M}\right)}{\bar{F}_{l e n}^{M}-1} & \bar{F}^{M}>a f_{L}\end{cases}
$$


where $\bar{F}_{l e n}^{M}$ is the maximum normalized muscle force achievable when the muscle fibre is lengthening, set to $1.8[41]$; and $S_{f}$ is a force-velocity shape factor set to $0.3[37$.

\subsubsection{Muscle Pennation Angle}

As shown in Figure 2.4, assuming straight, parallel, and coplanar muscle fibres, the height of the muscle $h^{M}$ is kept constant such that the muscle fibre pennation angle $\alpha^{M}$ changes with length:

$$
\begin{gathered}
h^{M}=L_{o}^{M} \sin \alpha_{o}^{M} \\
\alpha^{M}(t)= \begin{cases}0 & L^{M}(t)=0 \quad \text { or } \quad h^{M} / L^{M}(t) \leq 0 \\
\sin ^{-1}\left(h^{M} / L^{M}(t)\right) & 0<h^{M} / L^{M}(t)<1 \\
\cos ^{-1}(0.1) & h^{M} / L^{M}(t) \geq 1\end{cases}
\end{gathered}
$$

Values for $\alpha(t)$ are restricted between 0 and $\frac{\pi}{2}$ as exceeding this range represents states that are not physically achievable whereby muscle height exceeds muscle length or decreases to zero (see Figure 2.4), which can cause singularities during simulation. As such, restricting the upper range to $\cos ^{-1}(0.1)$, about $84^{\circ}$, ensures the equations do not become numerically stiff as the pennation angle approaches $\frac{\pi}{2}[42$.

The pennation angle is used to determine tendon length as a function of muscle length and pennation angle from the total musculotendon length $L^{M T}$, determined from musculoskeletal geometry:

$$
L^{T}=L^{M T}-L^{M} \cos \alpha^{M}
$$




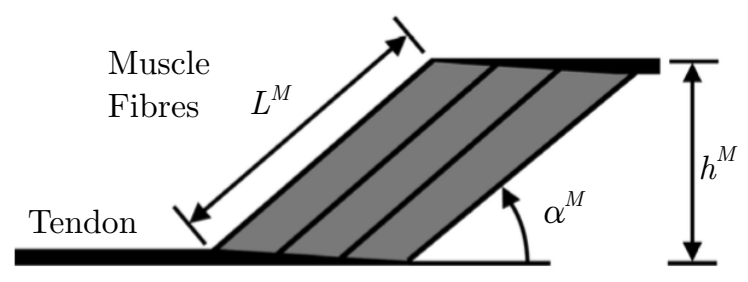

Figure 2.4: Simplified geometric representation of tendon and muscle fibres at pennation angle $\alpha$ for musculotendon modelling $[42$.

\subsubsection{Tendon Model}

Tendon consists of elastic tissue attached in series with muscle, making it responsible for load transfer to bone in order to initiate motion. Tendons are passive elements and act as a nonlinear elastic spring that only carries loads at lengths greater than the tendon slack length $L_{s}^{T}$ 222. As shown in Figure 2.3 $\mathrm{C}$, the tendon's force-strain relationship is represented by an exponential function at the nonlinear toe region, followed by a linear function thereafter [41]:

$$
\bar{F}^{T}= \begin{cases}\frac{\bar{F}_{t o e}^{T}}{e^{k_{t} o e}-1}\left(e^{k_{t o e} \varepsilon^{T} / \varepsilon_{t o e}^{T}}-1\right) & \varepsilon^{T} \leq \varepsilon_{\text {toe }}^{T} \\ k_{l i n}\left(\varepsilon^{T}-\varepsilon_{\text {toe }}^{T}\right)+\bar{F}_{\text {toe }}^{T} & \varepsilon^{T}>\varepsilon_{\text {toe }}^{T}\end{cases}
$$

where

$$
\begin{gathered}
\bar{F}^{T}=F^{T} / F_{i s o}^{\max } \\
k_{\text {toe }}=3 \\
k_{\text {lin }}=1.712 / \varepsilon_{0}^{T}
\end{gathered}
$$




$$
\begin{gathered}
\varepsilon^{T}=\frac{L^{T}-L_{s}^{T}}{L_{s}^{T}} \\
\varepsilon_{\text {toe }}^{T}=0.609 \varepsilon_{0}^{T} \\
\varepsilon_{0}^{T}=0.033 \\
\bar{F}_{\text {toe }}^{T}=0.33
\end{gathered}
$$

such that $\bar{F}^{T}$ is the tendon force normalized to maximum isometric force, $\varepsilon^{T}$ is tendon strain, $\varepsilon_{t o e}^{T}$ is tendon strain in the linear region corresponding to normalized forces greater than $\bar{F}_{\text {toe }}^{T}, k_{t o e}$ is an exponential shape factor, $k_{\text {lin }}$ is a linear scale factor, and $\varepsilon_{0}^{T}$ is the tendon strain due to maximum isometric force with respective relationships shown above 41.

\subsubsection{Rigid-tendon Simplification}

Recent work by Millard [42] which uses an inextensible tendon assumption has shown good agreement with the contraction dynamics model using an elastic tendon, as long as tendon slack length does not significantly exceed the optimal fibre length. With this assumption, a drastic increase in computational speed and simplicity can be achieved by reducing the first-order differential equation (Equation 2.16) to a kinematic model of the musculotendon actuator as shown below [42]:

$$
L^{M T}=L^{T}+L^{M} \cos \alpha^{M}
$$


The tendon length is assumed to be the tendon slack length $L_{s}^{T}$, with a zero velocity. Therefore, differentiating this with respect to time yields:

$$
V^{M T}=V^{M} \cos \alpha^{M}-L^{M} \dot{\alpha}^{M} \sin \alpha^{M}
$$

Muscle length and pennation angle are related by the constant height $h^{M}$ defined in Equation 2.18.

$$
h^{M}=L^{M} \sin \alpha^{M}
$$

Differentiating this with respect to time yields an expression of the pennation velocity:

$$
0=V^{M} \sin \alpha^{M}+L^{M} \dot{\alpha}^{M} \cos \alpha^{M}
$$

which can be solved for $\dot{\alpha}^{M}$ resulting in:

$$
\dot{\alpha}^{M}=-\frac{V^{M} \sin \alpha^{M}}{L^{M} \cos \alpha^{M}}
$$

Substituting this expression into Equation 2.30 yields an expression for $V^{M}$ :

$$
V^{M}=\frac{V^{M T}}{\cos \alpha^{M}+\tan \alpha^{M} \sin \alpha^{M}}
$$

In doing so, the force--velocity relationship can be readily computed by inverting Equation 2.16 with the addition of singularity avoidance constraints 45]. To derive $f_{V}\left(\dot{L}^{M}\right)$, the expression is modified such that $V^{M}$ can be linearly extrapolated when $F^{M}<0$ (concentric contraction), and $F^{M}>0.95 F_{l e n}^{M}$ (eccentric contraction), such that $f_{V}$ never decreases below 0 , or exceeds $F_{l e n}^{M}$. The inverted relationship, 
formulated by John [45] is as follows:

$$
f_{V}\left(\bar{V}^{M}\right)= \begin{cases}\frac{\bar{V}^{M}+1}{1+\frac{1}{S_{f}}} & \bar{V}^{M}<-1 \\ \frac{\bar{V}^{M}+1}{1-\frac{\bar{V}^{M}}{S_{f}}} & -1 \leq \bar{V}^{M}<0 \\ \frac{\left(2+\frac{2}{S_{f}}\right) \bar{V}^{M} \bar{F}_{l e n}^{M}+\bar{F}_{l e n}^{M}-1}{\left(2+\frac{2}{S_{f}}\right) \bar{V}^{M}+\bar{F}_{l e n}^{M}-1} & 0 \leq \bar{V}^{M}<\frac{10\left(\bar{F}_{l e n}^{M}-1\right)\left(0.95 \bar{F}_{l e n}^{M}-1\right)}{\left(1+\frac{1}{A_{f}}\right) \bar{F}_{l e n}^{M}} \\ \frac{\bar{F}_{l e n}^{M}}{20\left(\bar{F}_{l e n}^{M}-1\right)}\left[\frac{\left(1+\frac{1}{S_{f}}\right) \bar{F}_{l e n}^{M} \bar{V}^{M}}{10\left(\bar{F}_{l e n}^{M}-1\right)}+18.05 \bar{F}_{l e n}^{M}-18\right] & \frac{10\left(\bar{F}_{l e n}^{M}-1\right)\left(0.95 \bar{F}_{l e n}^{M}-1\right)}{\left(1+\frac{1}{S_{f}}\right) \bar{F}_{l e n}^{M}} \leq \bar{V}^{M}\end{cases}
$$

with parameters as defined in Section 2.3.3. For computational efficiency, the rigid tendon simplification is applied to the model developed in this study.

\section{$2.4 \quad$ Energy Expenditure Model}

The use of a muscle-driven biodynamic modelling approach has the added benefit of providing an accurate estimate of metabolic energy consumption through the direct computation of musculotendon forces and kinematics. In doing so, the effects of muscle redundancy and co-activation can be accounted for.

In general, an accurate muscle energetics model has important applications in forward-dynamic musculoskeletal studies, since energy minimization is often used as a criterion to predict muscle activity and coordination when performing a task [46].

The natural choice for modelling the relationship between muscle mechanics and energetics is through the use of Huxley's cross-bridge model [28], as it contains the framework for ATP hydrolysis. This model requires numerous parameters, whose values are uncertain particularly for human muscles, and it is therefore much simpler to use an empirical model with the Hill-type muscle mechanics model outlined in this chapter [46]. 
In order to compute muscle energetics from the force-producing properties of muscles, phenomenological models of muscle energy expenditure have been developed for use with the simple Hill-type models for muscle contraction, and have been shown to be useful for varying levels of complexity from simulating isolated muscle contractions to simulations of whole body locomotion [44, 46].

Following the traditional first law of thermodynamics approach to partitioning energy consumption in muscles:

$$
\dot{E}=\dot{H}+\dot{W}
$$

where the net change in internal energy, or total rate of energy expenditure $\dot{E}$, is represented by the heat generated $\dot{H}$ and rate of mechanical work $\dot{W}$ done by the muscle's contractile element. The work rate can be expressed as:

$$
\dot{W}=-F^{M} V^{M}
$$

Since positive work is done when muscles shorten, producing a negative contraction velocity, the negative sign ensures the sense is corrected. It is important to note that the work rate does not include work done by (or on) the series and parallel elastic elements of the musculotendon unit [44]. The energy stored in these springs is restorative and simply transfers between agonist-antagonist groups.

The heat generated by muscle can be expressed as:

$$
\dot{H}=m^{M}\left(\dot{h}_{A M}+\dot{h}_{S L}\right)
$$

where muscle mass $m^{M}$ is computed as follows:

$$
m^{M}=\rho^{M} L_{o}^{M} \frac{F_{i s o}^{\max }}{\sigma^{M}}
$$


such that $\rho^{M}$ is the density of muscle tissue, assumed to be $1059.7 \mathrm{~kg} / \mathrm{m}^{3}$ [44], and $\sigma^{M}$ is the specific tension for muscle, assumed to be $0.25 \mathrm{MPa}$ [44]. Dividing the maximum isometric force by the specific tension provides an estimate of the muscle's physiological cross-sectional area. The heat rate is normalized to the muscle's mass, expressed as the sum of $\dot{h}_{A M}$ and $\dot{h}_{S L}$ representing activation and maintenance, and shortening and lengthening heat rates in $\mathrm{W} / \mathrm{kg}$. These terms are detailed in the following subsections according to Umberger's formulation [44].

\subsubsection{Activation and Maintenance Heat Rate}

Activation and maintenance heat rates are represented as a combined term, which is a function of the muscle's composition of slow- (ST) and fast-twitch (FT) fibres as shown below. For mammalian muscles, this relationship has been empirically determined to be a linear function [44]:

$$
\dot{h}_{A M}=128 \mu_{F T}+25
$$

where $\dot{h}_{A M}$ is in $\mathrm{W} / \mathrm{kg}$ and $\mu_{F T}$ represents the proportion of fast-twitch fibres in a given musculotendon unit. These quantities are tabulated for the musculotendon units of interest in this study in Table 2.1.

\subsubsection{Shortening and Lengthening Heat Rate}

As muscle fibres shorten during contraction, the additional heat rate can be modelled empirically as a function of a coefficient $\alpha_{S}$ and contraction velocity $V^{M}$, where $\alpha_{S}$ varies inversely with $V^{M}$. It has been shown that slow twitch fibres at maximal $V^{M}$ have much larger heat rates as compared to fast-twitch fibres, resulting in the 
following relationships with units in s [44]:

$$
\begin{aligned}
\alpha_{S(S T)} & =\frac{100}{\bar{V}_{\max -S T}^{M}} \\
\alpha_{S(F T)} & =\frac{153}{\bar{V}_{\max -F T}^{M}}
\end{aligned}
$$

where $\bar{V}_{\max -S T}^{M}$ and $\bar{V}_{\max -F T}^{M}$ are the maximum normalized contraction velocities for slow- and fast-twitch fibres, assumed to be $10 \mathrm{~s}^{-1}$ and $25 \mathrm{~s}^{-1}$ respectively from in vivo estimates of human muscle [44]. Hence, the shortening heat rate in $\mathrm{W} / \mathrm{kg}$ is given by 44 :

$$
\dot{h}_{S L}=-\alpha_{S(S T)} \bar{V}^{M}\left(1-\mu_{F T}\right)-\alpha_{S(F T)} \bar{V}^{M} \mu_{F T}
$$

\section{Lengthening}

Heat rates during muscle lengthening have been experimentally determined to be proportional to $V^{M}$ by applying a scalar multiple of $\alpha_{S(S T)}$ such that 44 :

$$
\alpha_{L}=4 \alpha_{S(S T)}
$$

so that the heat rate for lengthening can then be expressed as [44]:

$$
\dot{h}_{S L}=\alpha_{L} \bar{V}^{M}
$$

\subsubsection{Scaling Factors}

As with the force-length relationship in contraction dynamics, heat rates $\dot{h}_{A M}$ and $\dot{h}_{S L}$ scale with muscle activation such that these quantities are maximum at $L_{o}^{M}$ 
and decrease as lengths deviate from $L_{o}^{M}$. As such, the normalized force-length relationship $f_{L}$ can be applied as a scaling factor when $L^{M}>L_{o}^{M}$ 44.

Motor unit recruitment depends both on fibre type, and on the task being performed, making it nonlinear and asynchronous in nature as activation varies. As such, while muscle force and length may remain constant, local oscillations in force and length can result in changes in $\dot{h}_{A M}$ and $\dot{h}_{S L}$ at low activation levels. To account for these changes, a scaling factor, $\xi$, is defined, expressed as a function of neural excitation $u$ and muscle activation $a$ [44]:

$$
\xi= \begin{cases}u & u>a \\ (u+a) / 2 & u \leq a\end{cases}
$$

Empirically, $\xi$ has been shown to scale with $\dot{h}_{A M}$ by a factor of $\xi^{0.6}$, while $\dot{h}_{S L}$ can be scaled by $\xi^{2.0}$ for shortening velocities, and $\xi$ for lengthening [44], consistent with those used in the force-velocity relationships in contraction dynamics.

Finally, depending on the pathway used for synthesizing ATP (aerobic, anaerobic, or breakdown of phosphocreatine), the number of ATP molecules produced differs, thereby influencing heat generation. To account for these, a scaling factor $\zeta$ is introduced, where $\zeta=1.0$ for anaerobic conditions, and $\zeta=1.5$ for aerobic conditions 44 . 


\subsubsection{Total Energy Rate}

Combining the various heat and work relationships with the activation and ATP synthesis scaling factors, the total energy rate in $\mathrm{W}$ is obtained by:

$$
\text { if } \begin{aligned}
& L^{M} \leq L_{o}^{M} \\
& \qquad \begin{aligned}
\dot{E} & =m^{M} \xi^{0.6} \zeta \dot{h}_{A M} \\
& + \begin{cases}m^{M} \xi^{2.0} \zeta\left[-\alpha_{S(S T)} \bar{V}^{M}\left(1-\mu_{F T}\right)-\alpha_{S(F T)} \bar{V}^{M} \mu_{F T}\right] & \bar{V}^{M} \leq 0 \\
m^{M} \xi \zeta \alpha_{L} \bar{V}^{M} & \bar{V}^{M}>0\end{cases} \\
& -F^{M} V^{M}
\end{aligned}
\end{aligned}
$$

$$
\text { if } \begin{aligned}
L^{M} & >L_{o}^{M} \\
\dot{E} & =m^{M} \xi^{0.6} \zeta\left(0.4+0.6 f_{L}\right) \dot{h}_{A M} \\
& + \begin{cases}m^{M} \xi^{2.0} \zeta f_{L}\left[-\alpha_{S(S T)} \bar{V}^{M}\left(1-\mu_{F T}\right)-\alpha_{S(F T)} \bar{V}^{M} \mu_{F T}\right] & \bar{V}^{M} \leq 0 \\
m^{M} \xi \zeta \alpha_{L} f_{L} \bar{V}^{M} & \bar{V}^{M}>0\end{cases} \\
& -F^{M} V^{M}
\end{aligned}
$$

\subsection{Musculoskeletal Geometry}

In order to propagate the contraction dynamics model in time, accurate musculotendon lengths $L^{M T}$, and moment arms $r^{M}$ are required, which are a function of joint angles. Delp's lower extremity model [26], illustrated in Figure 2.5, is often considered the standard for use with research in gait analysis and optimal control techniques, as it is developed from numerous studies of anatomic data, thereby representing mean values of muscle coordinates and wrapping geometry, but retaining plausible 
anatomical and anthropometric dimensions [47]. The model considers the changes in musculotendon lengths and moment arms as a function of joint angles in 3DOF at the hip and ankle joints and 1DOF at the knee joint, and models the elaborate geometry associated with the wrapping of the musculotendon unit around bone and soft tissue. This is especially critical for biarticulate muscles - those spanning multiple joints and thereby generating torque about those joints, such as the hamstrings, which have lengths and moment arms that are multivariate functions of more than one joint angle. The geometry for the musculotendon units of interest have been numerically
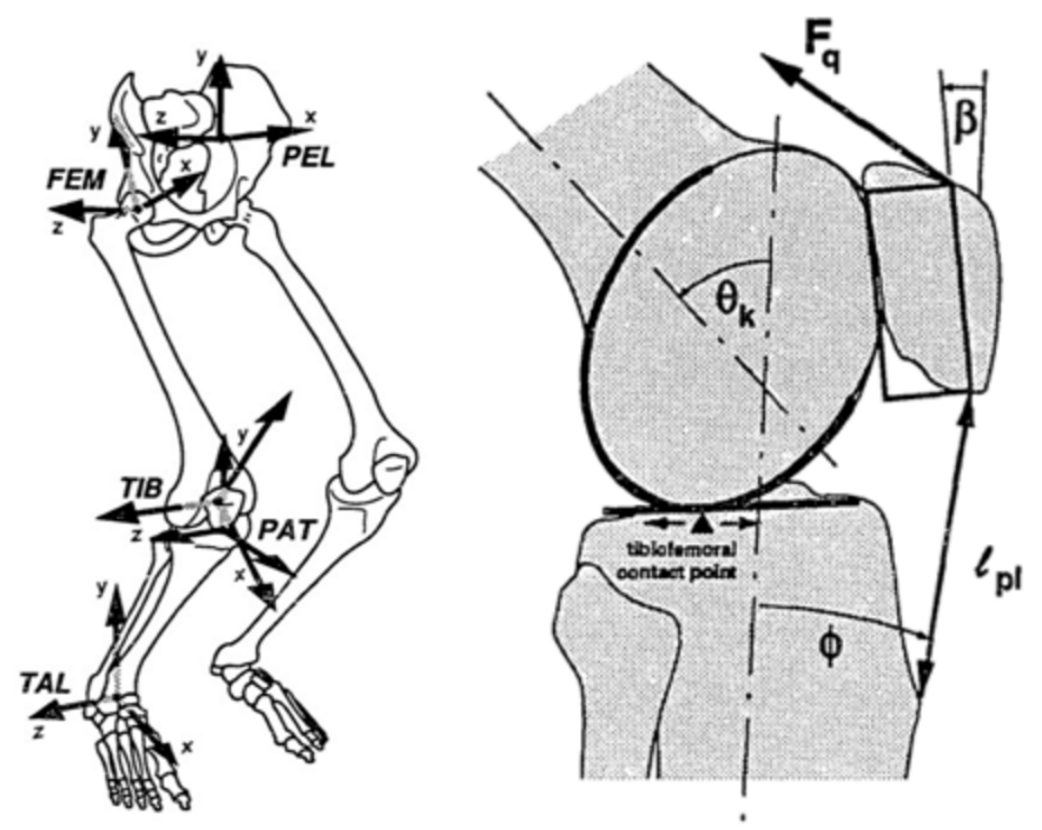

Figure 2.5: Delp's lower extremity model (left) and sagittal-plane knee geometry model for determining kinematics and moment arms (right) [26].

modelled by Menegaldo 47] based on Delp's lower extremity model. The model uses a set of differentiable regression equations to ensure continuously-differentiable relationships to facilitate simulation of nonlinear state-space equations. In addition to ankle, knee, and hip flexions in the sagittal plane, the model accounts for hip rotation (transverse plane), hip adduction-abduction (frontal plane), ankle rotation (transverse plane), and ankle pronation-supination (frontal plane). The neutral 
configuration $\left(0^{\circ}\right)$ was assumed for the generalized coordinates associated with these additional DOF, resulting in the simplified model depicted in Equations 2.49 2.53 and Table 2.5. The model requires ankle, knee, and hip joint angles in degrees relative to each segment as defined below and illustrated in Figure 2.2.

Hip angle $\left(\theta_{H}\right):-10^{\circ}$ extension to $+95^{\circ}$ flexion relative to vertical Knee angle $\left(\theta_{K}\right):-120^{\circ}$ flexion to $0^{\circ}$ full extension Ankle angle $\left(\theta_{A}\right):-30^{\circ}$ plantar flexion to $+30^{\circ}$ dorsiflexion

The joint angle conversions between modelling conventions are further described in Section 3.4.1. The simplified equations for the various generalized coordinates $\left(\theta_{A}, \theta_{K}\right.$, $\left.\theta_{H}\right)$ used to compute musculotendon lengths $L^{M T}$ and moment arms $r^{M}$, derived from Menegaldo's work [47] are as follows. Kinematics for monoarticular musculotendon units which are a function of one generalized coordinate $f\left(\theta_{1}\right)$ can be expressed as:

$$
\begin{aligned}
& L^{M T}, r_{1}^{M}\left(\theta_{1}\right)=a_{1}+a_{2} \theta_{1} \\
& L^{M T}, r_{1}^{M}\left(\theta_{1}\right)=a_{1}+a_{2} \theta_{1}+a_{3} \theta_{1}^{2} \\
& L^{M T}, r_{1}^{M}\left(\theta_{1}\right)=a_{1}+a_{2} \theta_{1}+a_{3} \theta_{1}^{2}+a_{4} \theta_{1}^{3}
\end{aligned}
$$

Similarly, kinematics for biarticular musculotendon units which are a function of two generalized coordinates $f\left(\theta_{1}, \theta_{2}\right)$ can be expressed as:

$$
\begin{aligned}
& L^{M T}, r_{1}^{M}, r_{2}^{M}\left(\theta_{1}, \theta_{2}\right)=a_{1}+a_{2} \theta_{1}+a_{3} \theta_{2}+a_{4} \theta_{1}^{2}+a_{5} \theta_{2}^{2} \\
& L^{M T}, r_{1}^{M}, r_{2}^{M}\left(\theta_{1}, \theta_{2}\right)=a_{1}+a_{2} \theta_{1}+a_{3} \theta_{2}+a_{4} \theta_{1}^{2}+a_{5} \theta_{2}^{2}+a_{6} \theta_{1}^{3}+a_{7} \theta_{2}^{3}
\end{aligned}
$$

where moment arms $r_{n}^{M}$ correspond to their respective generalized coordinate $\theta_{n}$. The joint torque contributions for joint $j$ from each musculotendon unit $i$ can then 
be computed as the product of its respective tendon force and the moment arm for joint $j$ :

$$
T_{j}=\sum_{i=1}^{7} F_{i}^{T} r_{i, j}^{M}
$$

\subsection{Rigid Body Dynamic Equations of Motion}

\subsubsection{Model Description}

A three-segment inverted pendulum model is selected to model the musculoskeletal dynamic model actuated by the seven major musculotendon units of the lowerextremity selected in this study (see Figure 2.2). This assumes that prescribed boat deck motions in surge, heave, and rotation about pitch, $\dot{x}, \dot{y}$, and $\dot{\theta}_{s}$, are completely known, and that the three segments of the human body are actuated about the ankle, knee, and hip joints exclusively in the sagittal plane, as shown in Figure 2.6.

Anthropometric parameters, shown in Table 2.3, are selected such that body segment lengths, $l$ are expressed as a fraction of body height $H$; segment masses $m$ are expressed as a fraction of total body mass $M$, and centre of mass locations $k$ are expressed as a fraction of segment length from the proximal joint. Inertial properties are computed from the radius of gyration $r_{g}$ about the CoM as a fraction of segment length such that the inertia for each link about its CoM can be computed as [20]:

$$
I=m r_{g}^{2}
$$


Table 2.2: Musculotendon geometry model, simplified from Menegaldo [4]. Moment arms reflect generalized coordinates in the order they appear; coefficients listed are as shown in their respective equations.

\begin{tabular}{|c|c|c|c|c|c|c|c|}
\hline \multicolumn{2}{|c|}{ Parameter } & \multicolumn{2}{|r|}{$L^{M T}$} & \multicolumn{2}{|r|}{$r_{1}^{M}$} & \multicolumn{2}{|r|}{$r_{2}^{M}$} \\
\hline Muscle & Gen. Coord. & Eqn. & Coef. & Eqn. & Coef. & Eqn. & Coef. \\
\hline GLU & $f\left(\theta_{H}\right)$ & 2.50 & $\begin{array}{c}1.9920 \mathrm{e}-01 \\
7.8172 \mathrm{e}-04 \\
-2.6248 \mathrm{e}-06\end{array}$ & 2.49 & $\begin{array}{c}-4.6424 \mathrm{e}-02 \\
4.1756 \mathrm{e}-04\end{array}$ & - & - \\
\hline $\mathrm{RF}$ & $f\left(\theta_{H}, \theta_{K}\right)$ & 2.53 & $\begin{array}{c}4.0883 \mathrm{e}-01 \\
-6.5657 \mathrm{e}-04 \\
-7.6018 \mathrm{e}-04 \\
-4.9235 \mathrm{e}-06 \\
1.7649 \mathrm{e}-06 \\
4.1737 \mathrm{e}-08 \\
2.3524 \mathrm{e}-08\end{array}$ & 2.52 & $\begin{array}{r}4.2355 \mathrm{e}-02 \\
5.4937 \mathrm{e}-04 \\
-2.6219 \mathrm{e}-06 \\
-7.0711 \mathrm{e}-06 \\
-8.1646 \mathrm{e}-09\end{array}$ & 2.53 & $\begin{array}{c}4.7207 \mathrm{e}-02 \\
1.6816 \mathrm{e}-05 \\
-3.3832 \mathrm{e}-04 \\
-1.7342 \mathrm{e}-07 \\
-1.0729 \mathrm{e}-05 \\
-4.5999 \mathrm{e}-10 \\
-4.9849 \mathrm{e}-08\end{array}$ \\
\hline VAS & $f\left(\theta_{K}\right)$ & 2.51 & $\begin{array}{c}1.8367 \mathrm{e}-01 \\
-7.2127 \mathrm{e}-04 \\
1.0752 \mathrm{e}-06 \\
1.7009 \mathrm{e}-08\end{array}$ & 2.51 & $\begin{array}{c}4.5187 \mathrm{e}-02 \\
-1.3850 \mathrm{e}-04 \\
-6.2529 \mathrm{e}-06 \\
-2.8406 \mathrm{e}-08\end{array}$ & - & - \\
\hline $\mathrm{BF}$ & $f\left(\theta_{H}, \theta_{K}\right)$ & 2.53 & $\begin{array}{c}4.4624 \mathrm{e}-01 \\
7.9020 \mathrm{e}-04 \\
1.2610 \mathrm{e}-04 \\
4.4165 \mathrm{e}-06 \\
-9.9684 \mathrm{e}-06 \\
-4.3108 \mathrm{e}-08 \\
-3.9658 \mathrm{e}-08\end{array}$ & 2.52 & $\begin{array}{c}-5.1408 \mathrm{e}-02 \\
-5.1057 \mathrm{e}-04 \\
2.0330 \mathrm{e}-05 \\
7.3343 \mathrm{e}-06 \\
1.4408 \mathrm{e}-07\end{array}$ & 2.53 & $\begin{array}{c}-1.2799 \mathrm{e}-02 \\
-3.7578 \mathrm{e}-05 \\
7.5078 \mathrm{e}-04 \\
5.9150 \mathrm{e}-07 \\
-2.2937 \mathrm{e}-07 \\
-9.4552 \mathrm{e}-10 \\
-3.6403 \mathrm{e}-08\end{array}$ \\
\hline TA & $f\left(\theta_{A}\right)$ & 2.50 & $\begin{array}{c}3.0372 \mathrm{e}-01 \\
-7.0653 \mathrm{e}-04 \\
-1.1404 \mathrm{e}-06\end{array}$ & 2.50 & $\begin{array}{c}4.2111 \mathrm{e}-02 \\
1.2308 \mathrm{e}-04 \\
-4.3648 \mathrm{e}-06\end{array}$ & - & - \\
\hline GAS & $f\left(\theta_{K}, \theta_{A}\right)$ & 2.53 & $\begin{array}{c}4.5108 \mathrm{e}-01 \\
2.6319 \mathrm{e}-04 \\
7.2473 \mathrm{e}-04 \\
-1.6841 \mathrm{e}-07 \\
-1.5958 \mathrm{e}-06 \\
-2.0153 \mathrm{e}-09 \\
-3.3438 \mathrm{e}-08\end{array}$ & 2.53 & $\begin{array}{c}-1.7133 \mathrm{e}-02 \\
-6.0857 \mathrm{e}-05 \\
-7.6966 \mathrm{e}-17 \\
-5.7306 \mathrm{e}-07 \\
5.5347 \mathrm{e}-15 \\
-3.2352 \mathrm{e}-09 \\
1.0887 \mathrm{e}-19\end{array}$ & 2.52 & $\begin{array}{c}-4.1809 \mathrm{e}-02 \\
7.6311 \mathrm{e}-15 \\
1.7968 \mathrm{e}-04 \\
5.1625 \mathrm{e}-17 \\
5.7323 \mathrm{e}-06\end{array}$ \\
\hline SOL & $f\left(\theta_{A}\right)$ & 2.51 & $\begin{array}{c}2.9074 \mathrm{e}-01 \\
7.1550 \mathrm{e}-04 \\
-2.1727 \mathrm{e}-06 \\
-3.0692 \mathrm{e}-08\end{array}$ & 2.50 & $\begin{array}{c}-4.1314 \mathrm{e}-02 \\
2.4505 \mathrm{e}-04 \\
5.2709 \mathrm{e}-06\end{array}$ & - & - \\
\hline
\end{tabular}




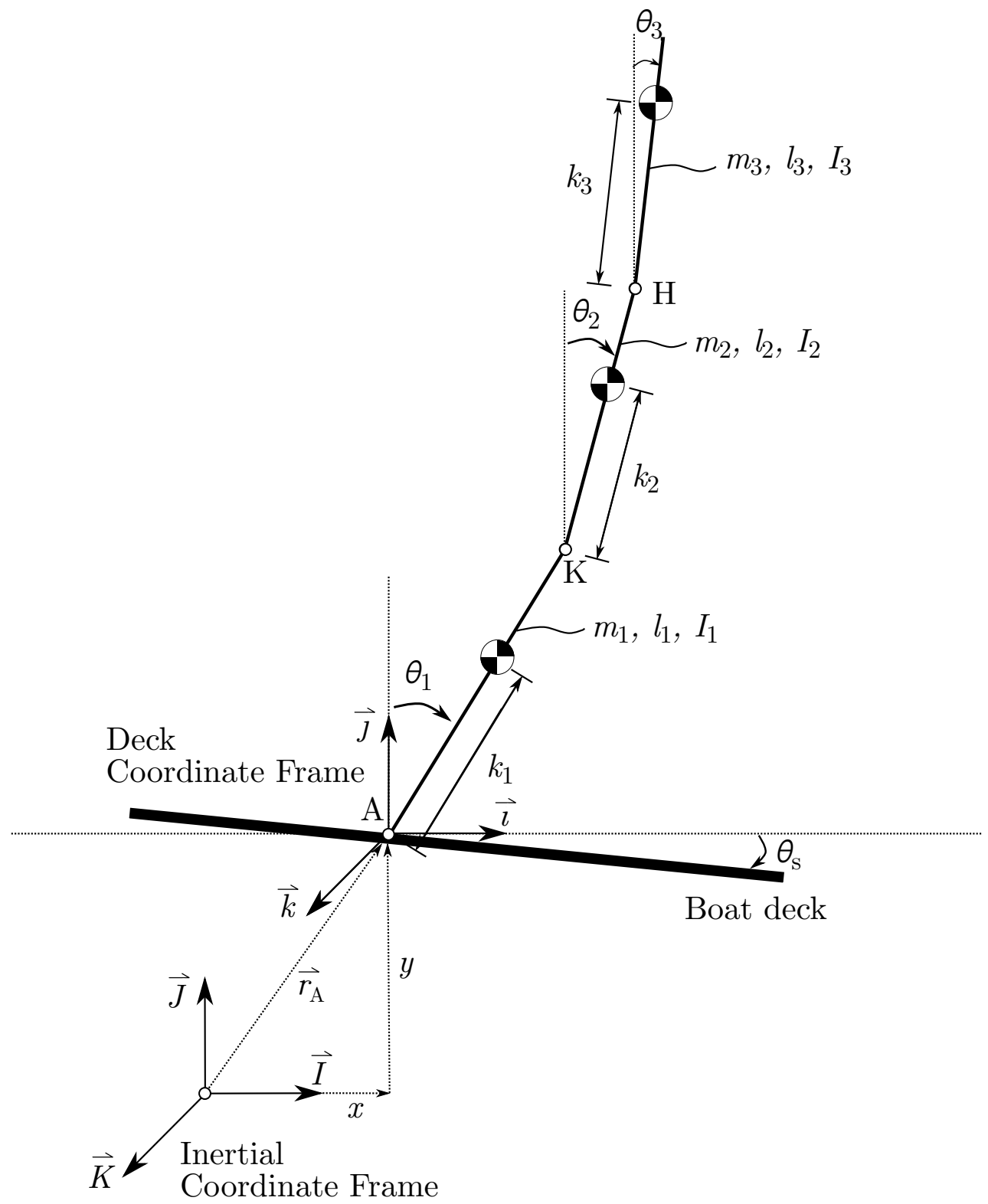

Figure 2.6: Three-link inverted pendulum model of leg, thigh, and HAT rotating about ankle, knee, and hip joints, respectively, under prescribed platform motions $\dot{x}, \dot{y}$, and $\dot{\theta}_{s}$. 
Table 2.3: Inertial parameters of three-link biodynamic model [20, p. 83-86].

\begin{tabular}{ccccc}
\hline Link & $m(\mathrm{~kg})$ & $l(\mathrm{~m})$ & $k(\mathrm{~m})$ & $r_{g}(\mathrm{~m})$ \\
\hline 1 & $0.0465 M$ & $0.246 \mathrm{H}$ & $0.567 l_{1}$ & $0.302 l_{1}$ \\
2 & $0.100 M$ & $0.245 H$ & $0.567 l_{2}$ & $0.323 l_{2}$ \\
3 & $0.678 M$ & $0.288 H$ & $0.626 l_{3}$ & $0.496 l_{3}$ \\
\hline
\end{tabular}

\subsubsection{Joint Positions and Velocities}

To derive the equations of motion, velocities of the joint centres, about which the limb segments rotate, are required in body-fixed coordinates for each segment. This is done by transforming the joint velocities in the global coordinate frame to the body-fixed coordinate frames using Euler angles as shown below.

\section{Joint Positions}

With respect to the global reference frame, joint positions can be derived as follows:

$$
\begin{aligned}
\vec{r}_{A}=\left[\begin{array}{l}
x \\
y
\end{array}\right] \\
\vec{r}_{K}=\vec{r}_{A}+\vec{r}_{K / A} \\
=\left[\begin{array}{l}
x+l_{1} \sin \theta_{1} \\
y+l_{1} \cos \theta_{1}
\end{array}\right]
\end{aligned}
$$




$$
\begin{aligned}
\vec{r}_{H} & =\vec{r}_{K}+\vec{r}_{H / K} \\
& =\left[\begin{array}{l}
x+l_{1} \sin \theta_{1}+l_{2} \sin \theta_{2} \\
y+l_{1} \cos \theta_{1}+l_{2} \cos \theta_{2}
\end{array}\right]
\end{aligned}
$$

\section{Joint velocities in global coordinates}

Differentiating the joint positions in time yields the joint velocities in the global reference frame:

$$
\begin{gathered}
\left\{\vec{v}_{A}\right\}^{g}=\left[\begin{array}{l}
\dot{x} \\
\dot{y}
\end{array}\right] \\
\left\{\vec{v}_{K}\right\}^{g}=\left[\begin{array}{l}
\dot{x}+l_{1} \dot{\theta}_{1} \cos \theta_{1} \\
\dot{y}-l_{1} \dot{\theta}_{1} \sin \theta_{1}
\end{array}\right] \\
\left\{\vec{v}_{H}\right\}^{g}=\left[\begin{array}{l}
\dot{x}+l_{1} \dot{\theta}_{1} \cos \theta_{1}+l_{2} \theta_{2} \cos \theta_{2} \\
\dot{y}-l_{1} \dot{\theta}_{1} \sin \theta_{1}-l_{2} \theta_{2} \sin \theta_{2}
\end{array}\right]
\end{gathered}
$$




\section{Joint velocities in body-fixed (local) coordinates}

Transforming the joint velocities to body-fixed (local) coordinates, defined with $\vec{\jmath}$ directed along the segment length from its proximal rotating joint yields:

$$
\begin{gathered}
\left\{\vec{v}_{A}\right\}^{l}=\left[\begin{array}{l}
\dot{x} \cos \theta_{1}-\dot{y} \sin \theta_{1} \\
\dot{x} \sin \theta_{1}-\dot{y} \cos \theta_{1}
\end{array}\right] \\
\left\{\vec{v}_{K}\right\}^{l}=\left[\begin{array}{l}
l_{1} \dot{\theta}_{1} \cos \left(\theta_{1}-\theta_{2}\right)+\dot{x} \cos \theta_{2}-\dot{y} \sin \theta_{2} \\
-l_{1} \dot{\theta}_{1} \sin \left(\theta_{1}-\theta_{2}\right)+\dot{x} \sin \theta_{2}+\dot{y} \cos \theta_{2}
\end{array}\right] \\
\left\{\vec{v}_{H}\right\}^{l}=\left[\begin{array}{l}
\dot{x} \cos \theta_{3}-\dot{y} \sin \theta_{3}+l_{1} \dot{\theta}_{1} \cos \left(\theta_{1}-\theta_{3}\right)+l_{2} \dot{\theta}_{2} \cos \left(\theta_{2}-\theta_{3}\right) \\
\dot{x} \sin \theta_{3}-\dot{y} \cos \theta_{3}-l_{1} \dot{\theta}_{1} \sin \left(\theta_{1}-\theta_{3}\right)-l_{2} \dot{\theta}_{2} \sin \left(\theta_{2}-\theta_{3}\right)
\end{array}\right]
\end{gathered}
$$

\subsubsection{Kinetic and Potential Energy}

Using standard formulations for rigid body dynamics, the kinetic and potential energy functions, $T$ and $V$ respectively, for the inverted pendulum model can be derived as follows with velocities in body-fixed (local) coordinates as defined above:

$$
\begin{array}{r}
T=\frac{1}{2} m_{1} v_{A}^{2}+\frac{1}{2}\left(I_{1}+m_{1} k_{1}^{2}\right) \dot{\theta}_{1}^{2}+m_{1} v_{A_{x}} \dot{\theta}_{1} k_{1}+\frac{1}{2} m_{2} v_{K}^{2}+\frac{1}{2}\left(I_{2}+m_{2} k_{2}^{2}\right) \dot{\theta}_{2}^{2} \\
+m_{2} v_{K_{x}} \dot{\theta}_{2} k_{2}+\frac{1}{2} m_{3} v_{H}^{2}+\frac{1}{2}\left(I_{3}+m_{3} k_{3}^{2}\right) \dot{\theta}_{3}^{2}+m_{3} v_{H_{x}} \dot{\theta}_{3} k_{3}
\end{array}
$$




$$
\begin{array}{r}
V=m_{1} g\left(k_{1} \cos \theta_{1}+y\right)+m_{2} g\left(l_{1} \cos \theta_{1}+k_{2} \cos \theta_{2}+y\right) \\
+m_{3} g\left(l_{1} \cos \theta_{1}+l_{2} \cos \theta_{2}+k_{3} \cos \theta_{3}+y\right)
\end{array}
$$

\subsubsection{Lagrange's Equations of Motion}

The equations of motion can then be derived using Lagrange's Equation:

$$
\frac{\mathrm{d}}{\mathrm{dt}}\left(\frac{\partial T}{\partial \dot{\theta}_{r}}\right)-\frac{\partial T}{\partial \theta_{r}}+\frac{\partial V}{\partial \theta_{r}}=Q_{\theta_{r}}
$$

where the generalized forces $Q_{\theta_{r}}$ are the sum of the musculotendon torques $T_{A}, T_{K}$, and $T_{H}$ about the ankle, knee, and hip respectively. The resulting equations, corresponding to the system's three degrees of freedom are:

$$
\begin{array}{r}
\ddot{\theta}_{1}\left[m_{1} k_{1}^{2}+m_{2} l_{2}^{2}+m_{3} l_{1}^{2}+I_{1}\right]+\ddot{\theta}_{2}\left[m_{2} l_{1} k_{2}+m_{3} l_{1} l_{2}\right] \cos \left(\theta_{1}-\theta_{2}\right) \\
+\ddot{\theta}_{3}\left[m_{3} l_{1} k_{3}\right] \cos \left(\theta_{1}-\theta_{3}\right)+\dot{\theta}_{2}^{2}\left[m_{2} l_{1} k_{2}+m_{3} l_{1} l_{2}\right] \sin \left(\theta_{1}-\theta_{2}\right) \\
+\dot{\theta}_{3}^{2}\left[m_{3} l_{1} k_{3}\right] \sin \left(\theta_{1}-\theta_{3}\right)+\ddot{x}\left[m_{1} k_{1}+m_{2} l_{1}+m_{3} l_{1}\right] \cos \theta_{1} \\
-\ddot{y}\left[m_{1} k_{1}+m_{2} l_{1}+m_{3} l_{1}\right] \sin \theta_{1}-g\left[m_{1} k_{1}+m_{2} l_{1}+m_{3} l_{1}\right] \sin \theta_{1} \\
-T_{K}=0 \\
\ddot{\theta}_{2}\left[m_{2} l_{1} k_{2}+m_{3} l_{1} l_{2}\right] \cos \left(\theta_{1}-\theta_{2}\right)+\ddot{\theta}_{2}\left[m_{2} k_{2}^{2}+m_{3} l_{2}^{2}+I_{2}\right] \\
+\ddot{\theta}_{3}\left[m_{3} k_{3} l_{2}\right] \cos \left(\theta_{2}-\theta_{3}\right)-\dot{\theta}_{1}^{2}\left[m_{2} k_{2} l_{1}+m_{3} l_{1} l_{2}\right] \sin \left(\theta_{1}-\theta_{2}\right) \\
+\dot{\theta}_{3}^{2}\left[m_{3} k_{3} l_{2}\right] \sin \left(\theta_{2}-\theta_{3}\right)+\ddot{x}\left[m_{2} k_{2}+m_{3} l_{2}\right] \cos \theta_{2} \\
-\ddot{y}\left[m_{2} k_{2}+m_{3} l_{2}\right] \sin \theta_{2}-g\left[m_{2} k_{2}+m_{3} l_{2}\right] \sin \theta_{2}-T_{K}+T_{H}=0
\end{array}
$$




$$
\begin{array}{r}
\ddot{\theta}_{1}\left[m_{3} l_{1} k_{3}\right] \cos \left(\theta_{1}-\theta_{3}\right)+\ddot{\theta}_{2}\left[m_{3} l_{2} k_{3}\right] \cos \left(\theta_{2}-\theta_{3}\right)+\ddot{\theta}_{3}\left[m_{3} k_{3}^{2}+I_{3}\right] \\
-\dot{\theta}_{1}^{2}\left[m_{3} k_{3} l_{1}\right] \sin \left(\theta_{1}-\theta_{3}\right)-\dot{\theta}_{2}^{2}\left[m_{3} l_{2} k_{3}\right] \sin \left(\theta_{2}-\theta_{3}\right)+\ddot{x}\left[m_{3} k_{3}\right] \cos \theta_{3} \\
-\ddot{y}\left[m_{3} k_{3}\right] \sin \theta_{3}-g\left[m_{3} k_{3}\right] \sin \theta_{3}-T_{H}=0
\end{array}
$$

These equations can be expressed in matrix form as:

$$
[M(\theta)]\{\ddot{\theta}\}=\{F(\theta, \dot{\theta}, \ddot{x}, \ddot{y})+T\}
$$

where $M$ represents the inertial matrix, $F$ represents the gravitational, Coriolis, and ground reaction force components, and $T$ contains the net muscle-applied joint torques. The system of equations can be integrated with respect to time to solve for the joint angular accelerations $\ddot{\theta}$ for use in kinematic analysis.

\subsection{Chapter Summary}

This chapter investigated various existing techniques in literature to model each of the required elements of the musculoskeletal dynamics modelling process. Modifications to these models were derived to suit the objective of characterizing postural stability in a 3DOF motion environment. This was done to facilitate numerical implementation for subsequent validation of this model with experimental data. 


\section{Chapter 3}

\section{Experiment Design and Instrumentation}

\subsection{Overview}

The objective of the neuromusculoskeletal model developed in this study is to estimate joint torques about the ankle, knee, and hip joints using a forward dynamic approach with neuromuscular stimuli, and predict muscle energetics in a motion environment. This chapter focuses on the data collection techniques and experiment design to validate the model developed.

Seven participants were recruited and asked to maintain balance on a motion base while neuromuscular activity, postural kinematics, and respiration metrics were measured. As inputs, the model requires neural excitation of the muscles derived from surface electromyography (sEMG), and prescribed kinematics of the motion base. To validate model output, joint kinematics were recorded using a spatial motion capture system, and respiration metrics were recorded for an independent measurement of metabolic energy output. The experimental setup is illustrated in Figure 3.1 and summarized in Table 3.1. 
Table 3.1: Experimental parameters for model validation.

\begin{tabular}{lcc}
\hline Instrument & Raw measurements & Computed Parameters \\
\hline Tape measure & Height & Segment lengths \\
Weighing scale & Weight & Segment masses, inertias \\
Motion platform & Positional trajectories & Joint kinematics \\
EMG amplifier & Surface EMG & Neural excitation \\
Motion capture & Marker trajectories & Joint kinematics \\
Respirometry & Respiratory volumes & Energy expenditure
\end{tabular}

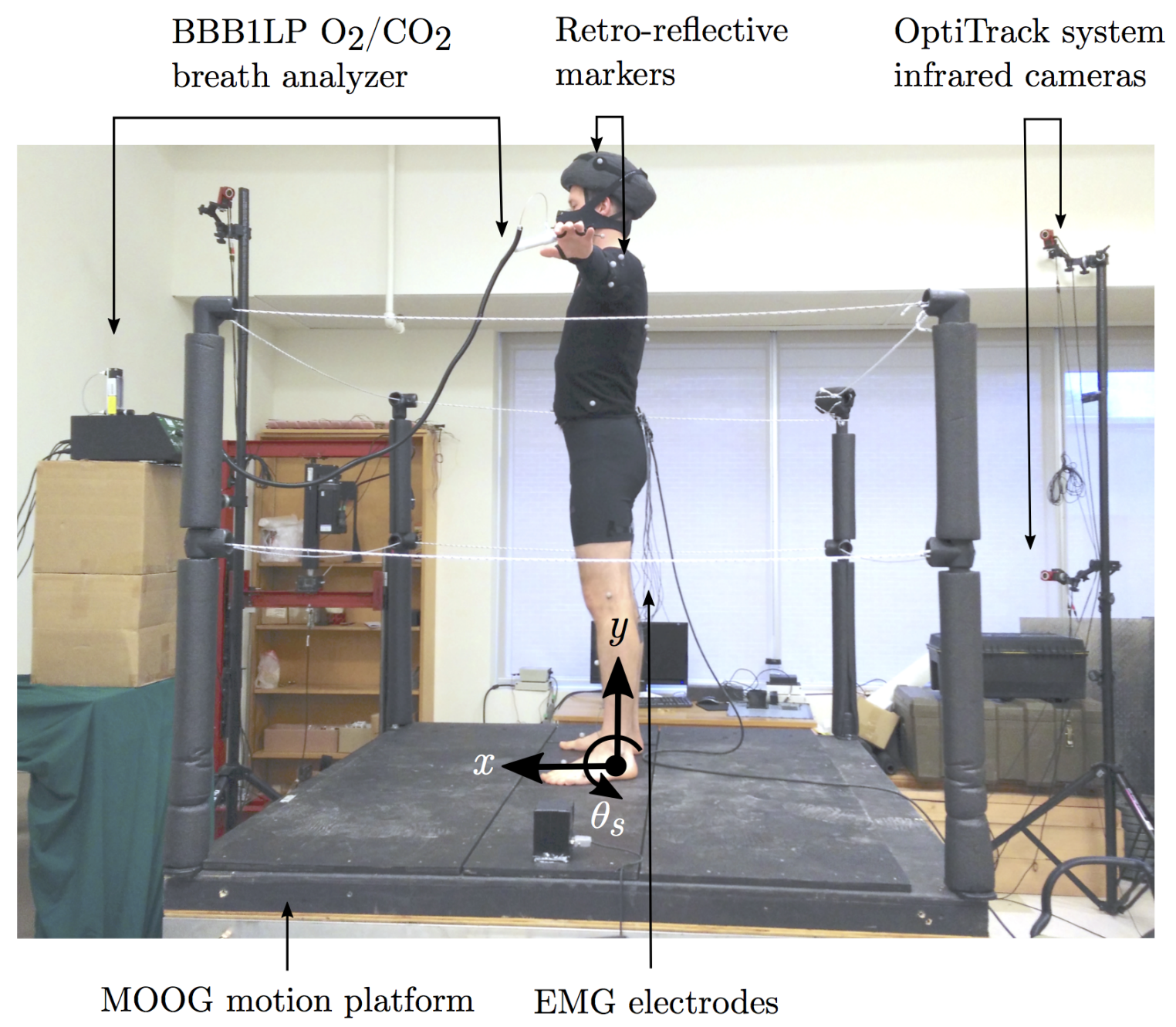

Figure 3.1: Experimental setup illustrating instrumentation and the 3DOF motions in surge, heave, and pitch corresponding to the $x, y$, and $\theta_{s}$ directions shown. 


\subsection{Anthropometric Parameters}

Anthropometric parameters define geometric and inertial properties of limb segments for the skeletal dynamic model developed in this study. These values were parameterized as functions of body mass and height as outlined in Section 2.6. In addition to body mass and height, segment lengths of the shank, thigh, and HAT, illustrated in Figure 3.2, were recorded for verification as follows 20,48]:

Shank length: lateral malleolus to lateral femoral epicondyle;

Thigh length: lateral femoral epicondyle to greater trochanter; and HAT length: greater trochanter to glenohumeral joint.

Palpable bony prominences approximating joint centres were selected as landmarks for measuring segment lengths as illustrated in Figure 3.2. These segment lengths were found to be within $\pm 5 \%$ of model-derived lengths and were therefore not used in the analysis. Anthropometric parameters for all seven participants are summarized in Table 3.2 .

Table 3.2: Measured and computed anthropometric parameters [51].

\begin{tabular}{cccccccc}
\hline Subject & Sex & Age & Height $(\mathrm{m})$ & Mass $(\mathrm{kg})$ & Shank $(\mathrm{m})$ & Thigh $(\mathrm{m})$ & HAT $(\mathrm{m})$ \\
\hline 1 & F & 30 & 1.55 & 46 & 0.38 & 0.38 & 0.45 \\
2 & M & 30 & 1.83 & 77 & 0.45 & 0.45 & 0.53 \\
3 & M & 27 & 1.75 & 76 & 0.43 & 0.43 & 0.50 \\
4 & M & 24 & 1.80 & 102 & 0.44 & 0.44 & 0.52 \\
5 & M & 25 & 1.85 & 74 & 0.45 & 0.45 & 0.53 \\
6 & F & 24 & 1.75 & 65 & 0.43 & 0.43 & 0.50 \\
7 & M & 23 & 1.75 & 75 & 0.43 & 0.43 & 0.50 \\
\hline
\end{tabular}




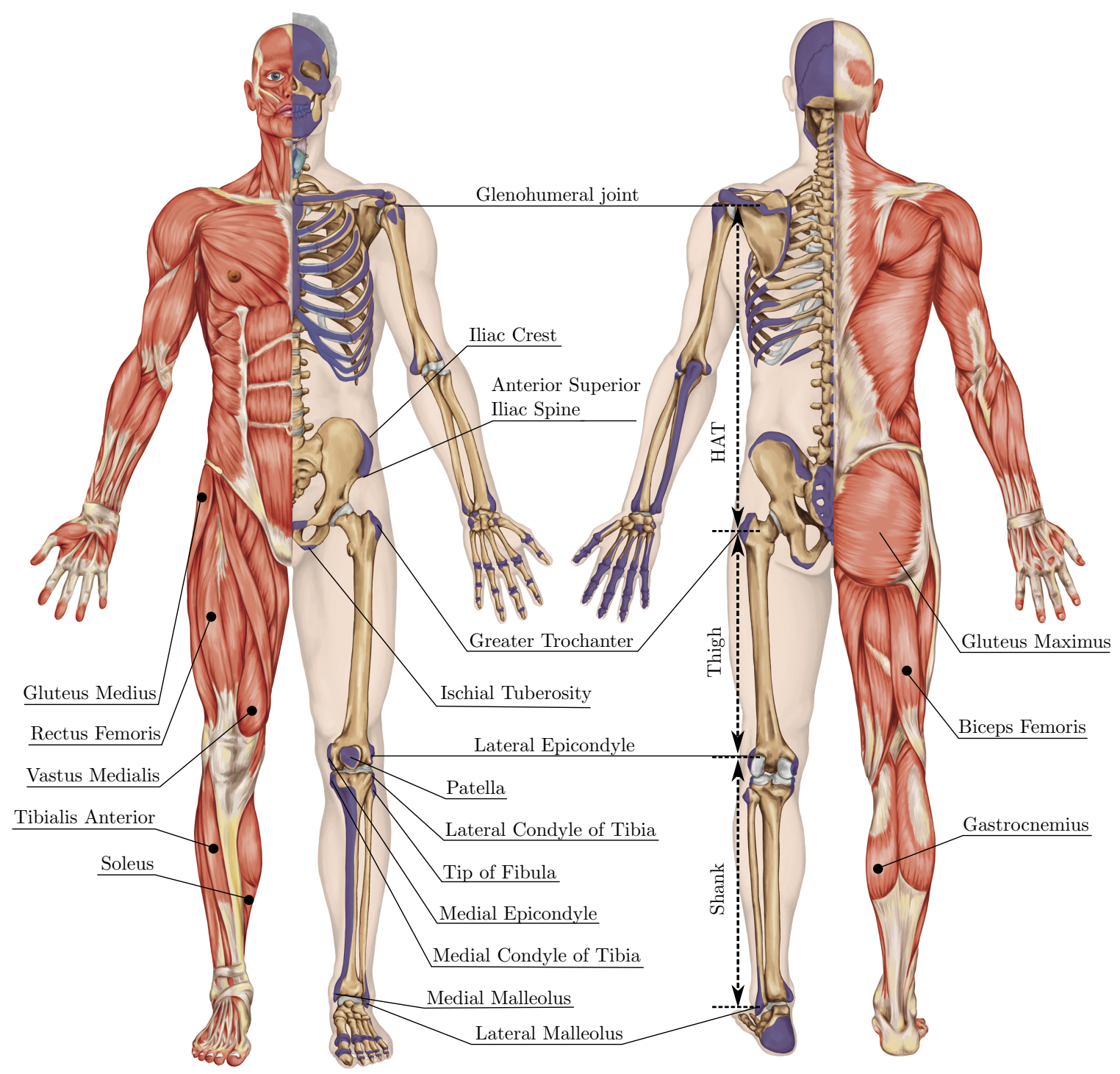

Figure 3.2: Palpable bony prominences in the lower extremity used to define segment lengths and locate muscle origin and insertion points. SENIAMrecommended [49] ideal muscle belly locations for electrode placements are identified by circular markers [50]. 


\subsection{Motion Platform}

Boat deck motion was simulated on a MOOG 6DOF-2000E Gough-Stewart platform [51]. As shown in Figure 3.3, the robot consists of two rigid frames separated by six linear actuators configured in parallel and is capable of motion in six degreesof-freedom. An inverse kinematic model allows the platform to be commanded by specifying positional data for each degree-of-freedom, for which actuator lengths are computed. When commanded by an input, the platform attempts to move to the

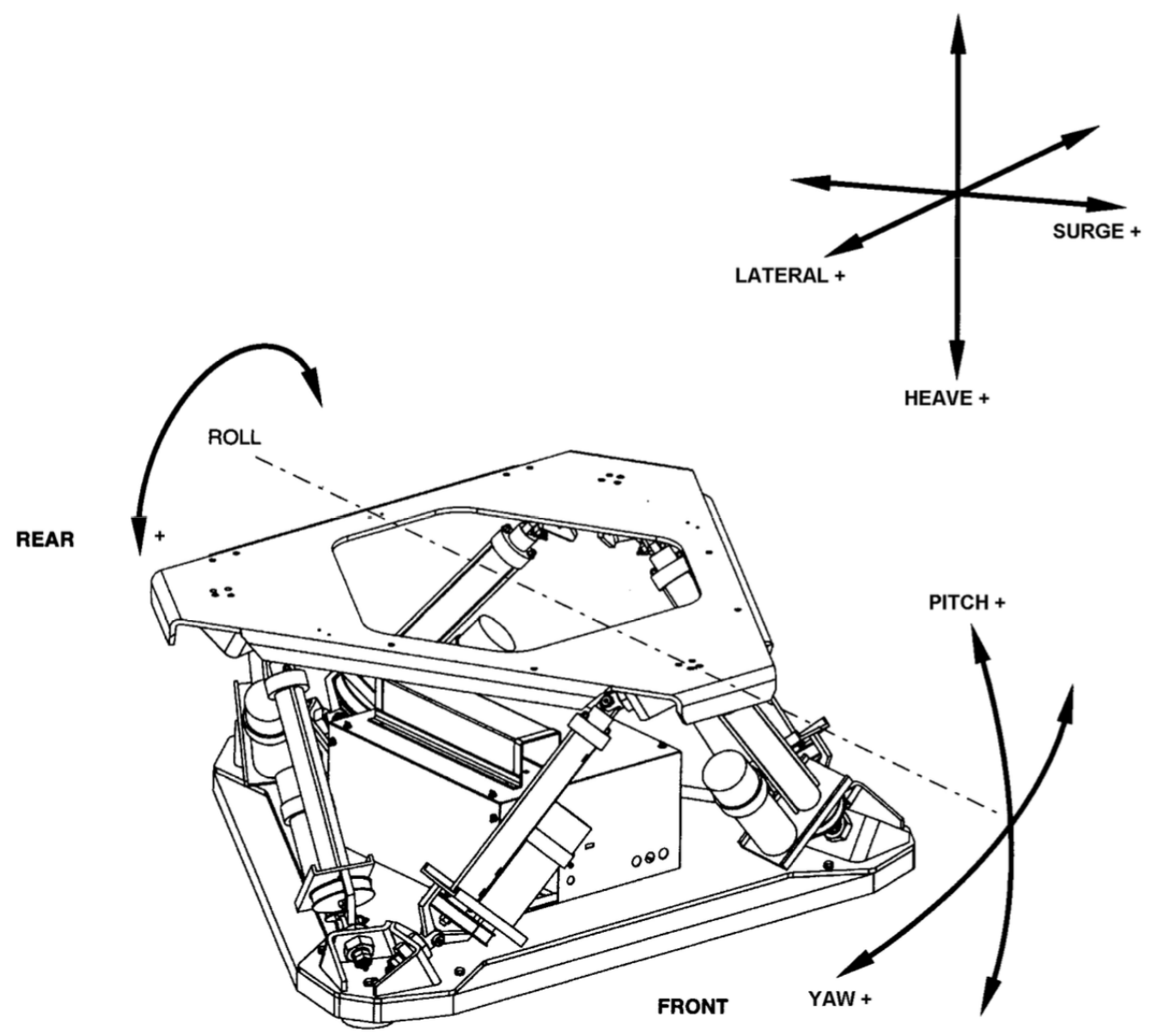

Figure 3.3: MOOG 6DOF-2000E Gough-Stewart platform [51.

target position instantaneously, within its kinematic bounds. Smooth motions can be produced by specifying discrete positional trajectories at $60 \mathrm{~Hz}$. The platform's 
software kinematic limits are listed in Table 3.3. It is important to note that these kinematic limits apply for each DOF individually. Generating motions with multiple DOF generally reduces the range of motion in all DOF due to the physical constraints of the linear actuators. Given the model's limitations to planar motion, the simulated

Table 3.3: MOOG platform software limits for degrees of freedom [51].

\begin{tabular}{cccc}
\hline DOF & Displacement & Velocity & Acceleration \\
\hline Pitch & $\pm 22 \mathrm{deg}$ & $\pm 30 \mathrm{deg} / \mathrm{s}$ & $\pm 500 \mathrm{deg} / \mathrm{s}^{2}$ \\
Roll & $\pm 21 \mathrm{deg}$ & $\pm 30 \mathrm{deg} / \mathrm{s}$ & $\pm 500 \mathrm{deg} / \mathrm{s}^{2}$ \\
Yaw & $\pm 22 \mathrm{deg}$ & $\pm 40 \mathrm{deg} / \mathrm{s}$ & $\pm 400 \mathrm{deg} / \mathrm{s}^{2}$ \\
Heave & $\pm 0.18 \mathrm{~m}$ & $\pm 0.3 \mathrm{~m} / \mathrm{s}$ & $\pm 0.5 \mathrm{~g}$ \\
Surge & $\pm 0.25 \mathrm{~m}$ & $\pm 0.5 \mathrm{~m} / \mathrm{s}$ & $\pm 0.6 \mathrm{~g}$ \\
Sway & $\pm 0.25 \mathrm{~m}$ & $\pm 0.5 \mathrm{~m} / \mathrm{s}$ & $\pm 0.6 \mathrm{~g}$ \\
\hline
\end{tabular}

motion was confined to move in three degrees-of-freedom: surge, heave, and pitch. While all motion types are possible, frigate data was used for validation for transferability to marine vessels and the HSC environment. Since the high-acceleration deck motions on HSC are not reproducible in the laboratory environment with the available motion platform, the frigate data was scaled to maximize accelerations according to the kinematic limitations of the MOOG platform, as shown in Table 3.3 . Practically, the platform's stroke was the limiting kinematic constraint, before any accelerations approaching the platform's limit could be produced. In order to take advantage of the kinematic limits, recorded frigate motion was therefore increased in frequency via a horizontal compression factor of 3 and displacement amplitude in heave was halved to increase velocity and acceleration in the combined 3DOF motion profile. The profiles were limited to $200 \mathrm{~s}$ in duration to reduce the effects of subject fatigue throughout the experimental trials.

Four motion profiles were generated for experimental trials. Chirp (frequency sweep) profiles in each of the 3DOF individually were generated with a duration of 
$200 \mathrm{~s}$ each, of the form:

$$
x(t)=A \sin \left[2 \pi\left(f_{0} t+\frac{k_{f}}{2} t^{2}\right)\right]
$$

where $f_{0}$ is the starting frequency, $k_{f}$ is the rate of frequency increase or chirp rate, and $A$ is the amplitude. In addition, a 100 s combined 3DOF motion profile was generated from frigate motion under superimposed wave conditions with varying frequency and amplitudes, of the form:

$$
x(t)=\sum_{i=1}^{n} A_{i} \sin \left(j \omega_{0} t+\phi_{i, R N D}\right)
$$

where $\omega_{0}$ is the frequency increment between harmonics, $A_{i}$ is the amplitude, and $\phi_{i, R N D}$ is the random phase component of the $i^{\text {th }}$ harmonic.

Chirp profiles were selected to investigate the frequency response of the neuromusculoskeletal system and to generate the largest accelerations possible for each individual DOF. The resulting motion profile parameters are shown in Table 3.4, and samples of the chirp profile in surge and a combined three-DOF profile are illustrated in Figure 3.4

Table 3.4: Motion profiles selected for experimental trials.

\begin{tabular}{ccccl}
\hline Profile & $A$ & $f_{0}$ & $k_{f}$ & Max. Acceleration \\
\hline Pitch Chirp & $0.064 \mathrm{rad}$ & $0.01 \mathrm{~Hz}$ & 0.00645 & $4.25 \mathrm{rad} / \mathrm{s}^{2}\left(243 \mathrm{deg} / \mathrm{s}^{2}\right)$ \\
Surge Chirp & $0.042 \mathrm{~m}$ & $0.01 \mathrm{~Hz}$ & 0.0093 & $5.77 \mathrm{~m} / \mathrm{s}^{2}(0.59 \mathrm{~g})$ \\
Heave Chirp & $0.03 \mathrm{~m}$ & $0.01 \mathrm{~Hz}$ & 0.0079 & $2.98 \mathrm{~m} / \mathrm{s}^{2}(0.3 \mathrm{~g})$ \\
& & & & Pitch: $1 \mathrm{rad} / \mathrm{s}^{2}\left(57 \mathrm{deg} / \mathrm{s}^{2}\right)$ \\
Combined & - & - & - & Surge: $0.55 \mathrm{~m} / \mathrm{s}^{2}(0.06 \mathrm{~g})$ \\
& & & & Heave: $0.59 \mathrm{~m} / \mathrm{s}^{2}(0.06 \mathrm{~g})$ \\
\hline
\end{tabular}



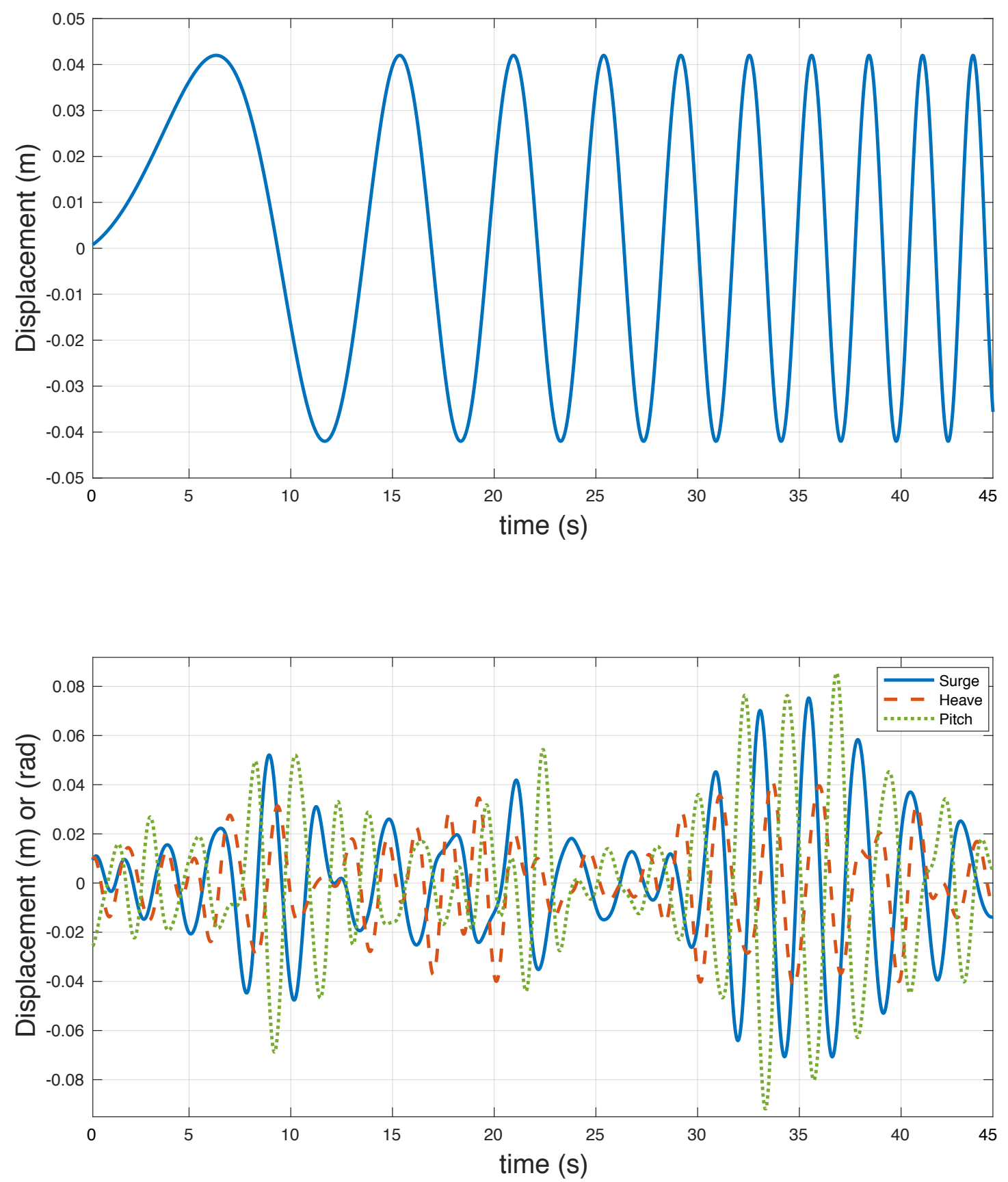

Figure 3.4: A sample of surge chirp (top) and combined 3DOF (bottom) motion profiles. 


\subsubsection{Post-processing Accelerations}

To derive accelerations of the generated motion for the derived equations of motion shown in Section 2.6, the motion profiles were resampled from $60 \mathrm{~Hz}$ to $100 \mathrm{~Hz}$ for consistency with other data sets. The chirp profiles trajectories were analytically differentiated as follows:

$$
\begin{gathered}
\frac{\mathrm{d} x}{\mathrm{~d} t}=2 \pi\left(f_{0}+k t\right) A \cos \left[2 \pi\left(f_{0} t+\frac{k_{f}}{2} t^{2}\right)\right] \\
\frac{\mathrm{d}^{2} x}{\mathrm{~d} t^{2}}=-4 \pi^{2}\left(f_{0}+k t\right)^{2} A \sin \left[2 \pi\left(f_{0} t+\frac{k_{f}}{2} t^{2}\right)\right]+2 \pi k A \cos \left[2 \pi\left(f_{0} t+\frac{k_{f}}{2} t^{2}\right)\right]
\end{gathered}
$$

where $x(t), \frac{\mathrm{d} x}{\mathrm{~d} t}$, and $\frac{\mathrm{d}^{2} x}{\mathrm{~d} t^{2}}$, represent the displacement, velocity, and acceleration respectively for pitch, surge, and heave in each of the chirp profiles. The combined 3DOF displacement motion trajectories were first fitted with fifth-order piecewise splines, then analytically differentiated to obtain velocities and accelerations for each of the 3DOF.

\subsection{Motion Capture}

A common approach for measuring kinematics of limb segments and other anatomical features is through the use of an optical motion capture system [24]. The motion capture system provides spatial positional trajectories of anatomical landmarks, which can be processed to extract joint angles of interest. In this study, an OptiTrack motion capture system was used with eight cameras surrounding the recording volume over the motion platform.

Well-placed cameras can significantly improve the tracking quality of the system. 
Since 3D marker positions are reconstructed from 2D camera views via triangulation, it is critical that each marker be visible by multiple cameras in any given position. As such, pairs of cameras were placed at each corner of the cubic capture volume, approximately equidistant to the participant, whereby the cameras forming each pair were placed at two elevations to maximize the collective field of view. Prior to calibrating the recording volume, any reflective surfaces, such as another camera's strobe ring, can be masked in software. This, however, creates blind spots in the cameras. Instead, any reflective features are physically masked or painted black wherever possible. The system of cameras is then calibrated by sweeping a reflective wand within the recording volume to identify blind spots for camera re-orientation. A calibration square containing three markers is then used to identify $x, y$, and $z$ coordinates to specify relative orientation of the recording volume's ground plane with respect to the cameras [52].

Participants were asked to wear a body-tight black velcro suit, on which 34 retroreflective spherical markers were placed at joint centres and other bony landmarks which remain relatively stationary with respect to the skin as limb segments are moved. The strategic placement of the markers allows the OptiTrack Arena software to fit a rigid body model to the participant, where each limb segment is modelled as a rigid body attached to spherical joints, as shown in Figure 3.5. The model is calibrated with a T-pose to verify visibility of all markers, where the participant is asked to face the positive $z$-axis with arms extended on either side. As shown in Figure 3.1, the positive $z$-axis of the OptiTrack system corresponds to the positive $x$, or surge, axis defined in the dynamic model [52]. The recorded motion capture data often contains gaps in the marker trajectories that need to be filled via interpolation. This is done by post-processing the data in Arena by manually specifying the identity of the dropped marker and applying an appropriate interpolation algorithm that smoothly fits the trajectory. The processed recording is then trajectorized and exported to a Biovision 

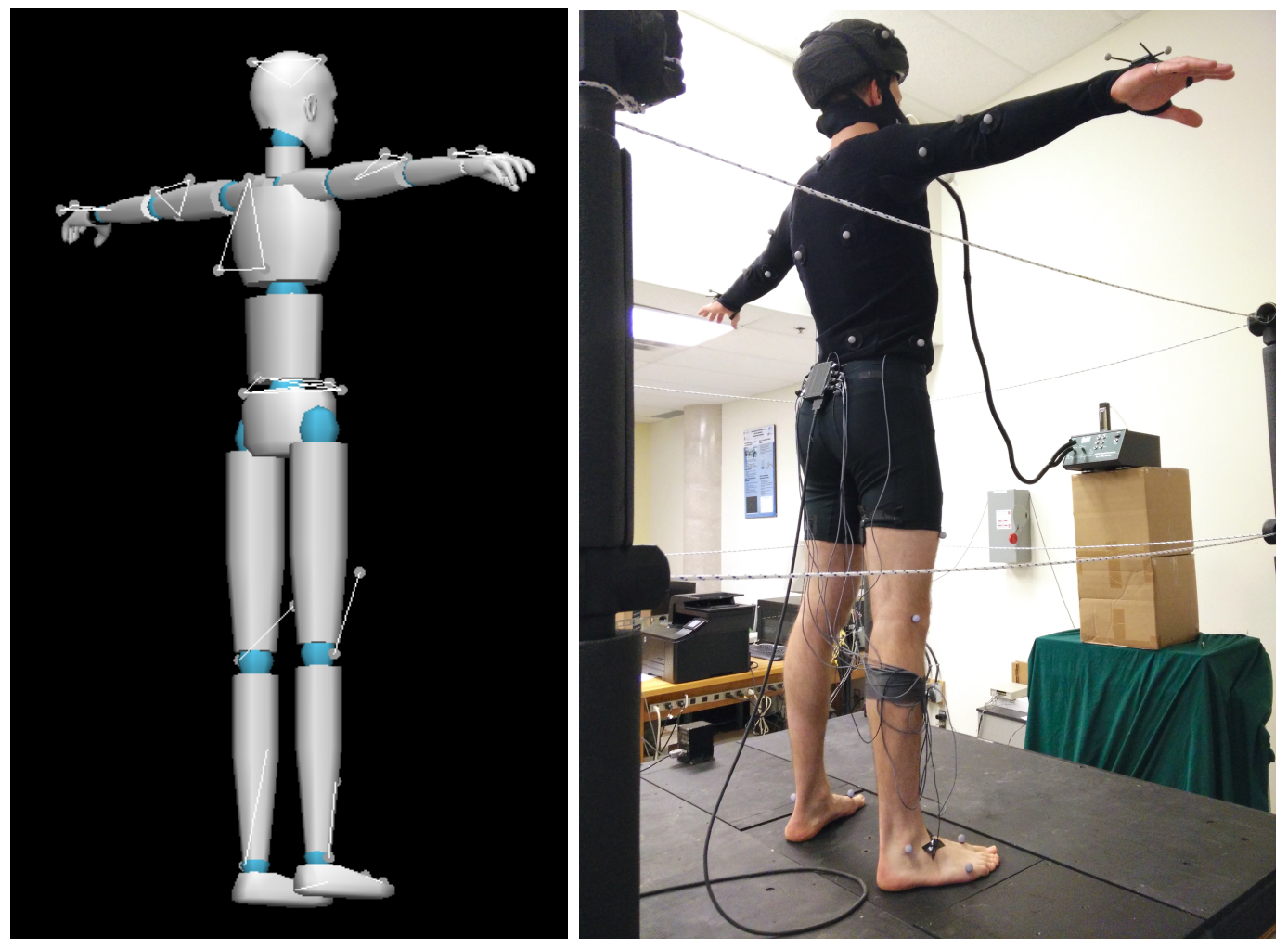

Figure 3.5: OptiTrack rigid-body skeleton model fit to participant's T-pose illustrating reflective marker placements and corresponding rigid-body limb segments. 
Hierarchy $(\mathrm{BVH})$ file containing the skeleton hierarchy of all the major joint centres and their positional trajectories in a cartesian coordinate system.

\subsubsection{Post-processing Joint Kinematics}

The BVH file contains a 3D skeleton hierarchy composed of 23 markers representing joint centres. Markers on the right leg and neck were selected to resolve the positional trajectories of the joint centres in the $y-z$ (sagittal) plane to an inverted pendulum model, as shown in Figure 3.6. Joint angles were then computed as the relative angle between line segments connecting these joint centres. Joint angles in the BVH convention require conversion, as shown below, for use with Menegaldo's 47 musculoskeletal geometry model convention as well as that described by the inverted pendulum skeletal dynamic (3IP) model developed in Section 2.6. Note that angles in the musculoskeletal geometry model and the BVH conventions are relative to limb segments in degrees, whereas those defined in the dynamic model are absolute with respect to the vertical in radians, but are shown here in degrees for convenience. Angles in the BVH convention can be converted to the musculoskeletal geometry [47] convention as follows:

$$
\begin{gathered}
\theta_{A}=90^{\circ}-\theta_{1, B} \\
\theta_{K}=\theta_{2, B}-180^{\circ} \\
\theta_{H}=180^{\circ}-\theta_{3, B}
\end{gathered}
$$



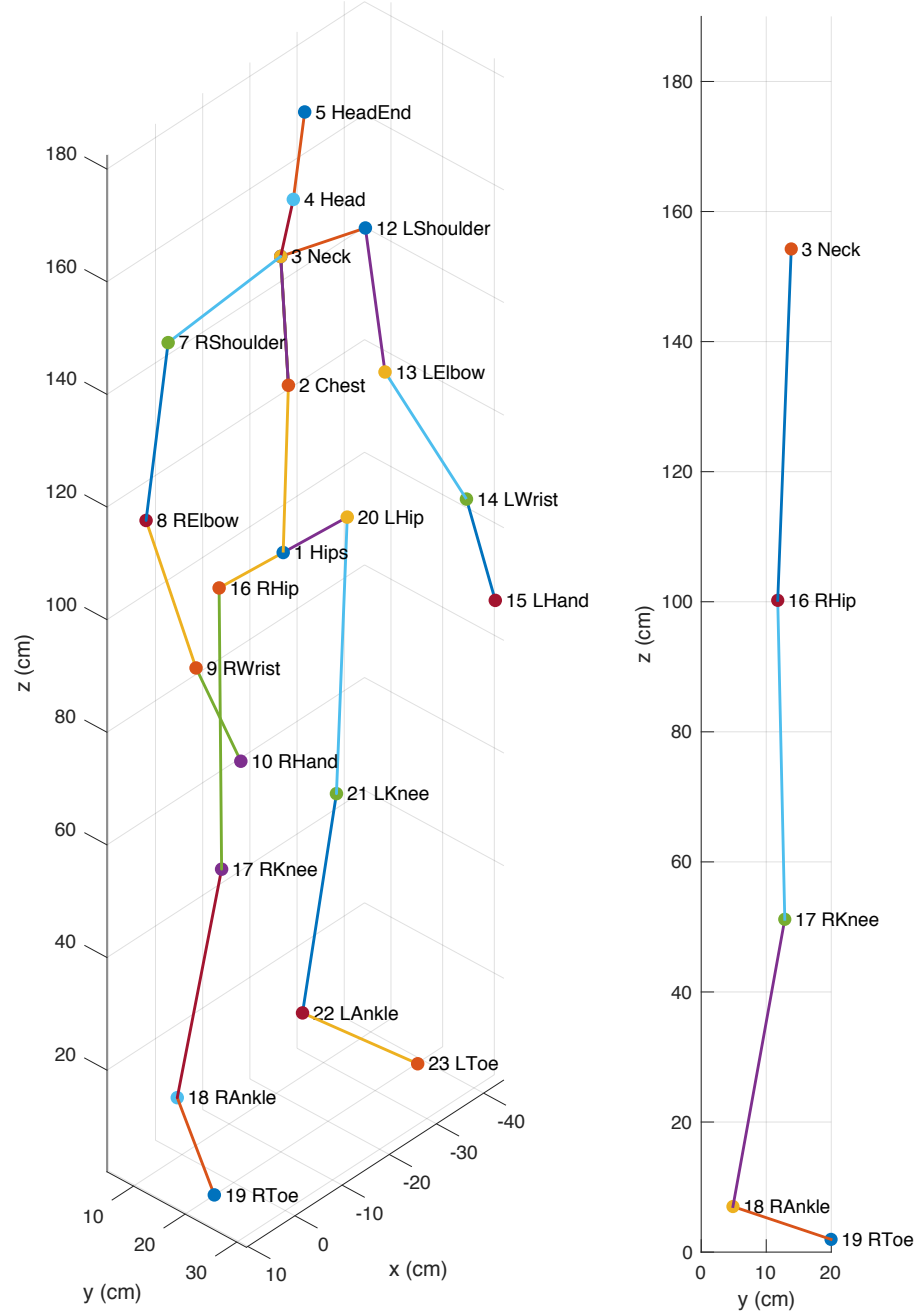

Figure 3.6: The 23 major joint centres defining the BVH skeleton (left), and the resolved planar inverted pendulum model (right). 


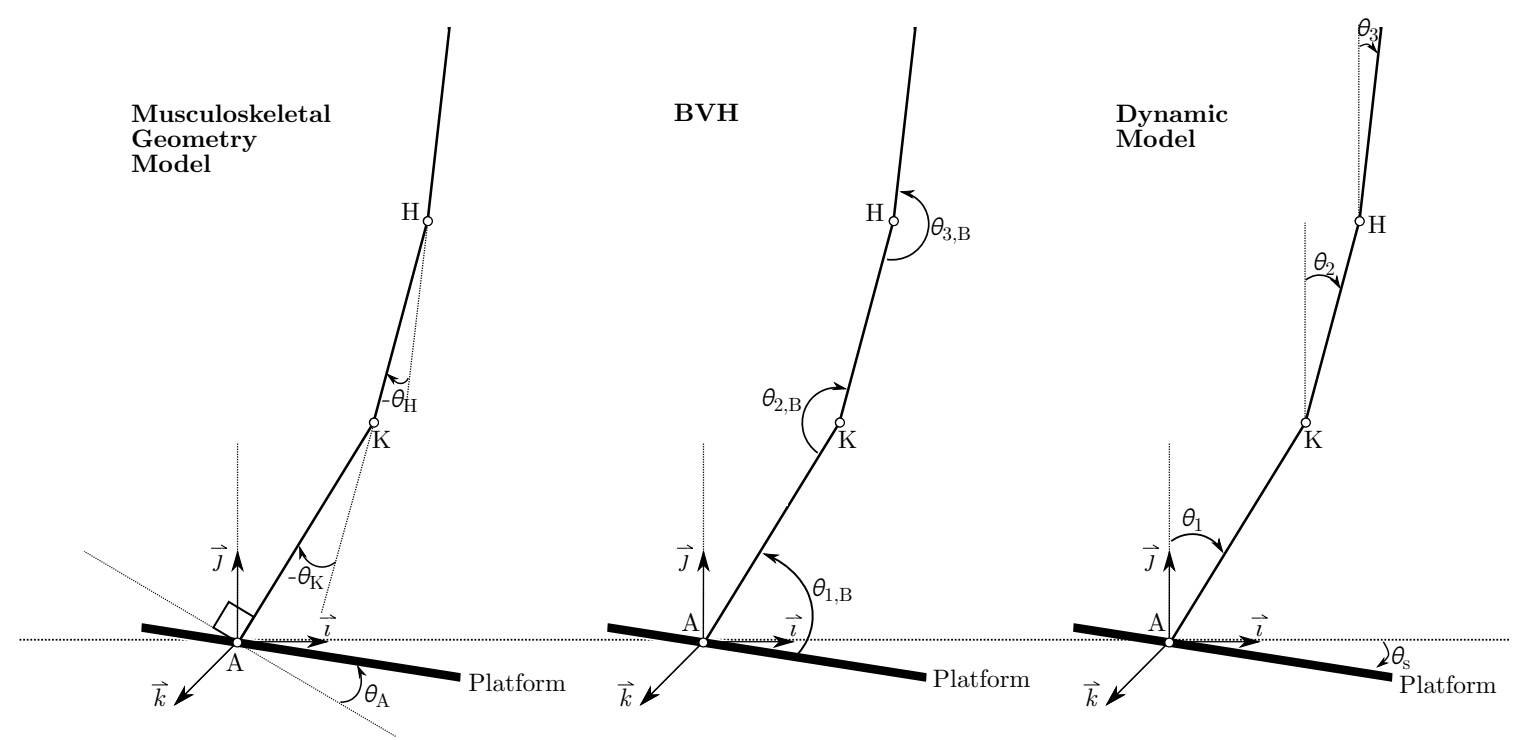

Figure 3.7: Joint angle conventions of the (left to right) musculoskeletal geometry model [47], BVH model, and 3IP dynamic model used in data post-processing.

Similarly, angles in the BVH convention can be converted to the dynamic model convention as follows:

$$
\begin{aligned}
& \theta_{1, B}=90^{\circ}-\theta_{1}+\theta_{s} \\
& \theta_{2, B}=180^{\circ}-\theta_{1}+\theta_{2} \\
& \theta_{3, B}=180^{\circ}+\theta_{2}-\theta_{3}
\end{aligned}
$$

The motion-capture-derived joint displacements contain noise in the form of electromagnetic interference (EMI) from the optoelectronic devices, and spatial precision in the digitizing process, both of which introduce random errors in the converted data which require smoothing [20, p. 64]. Joint displacement time histories were low pass filtered at a cutoff frequency of $6 \mathrm{~Hz}$ for chirp profiles, and $3 \mathrm{~Hz}$ for all other movements using a fourth-order zero-lag Butterworth filter, which is the preferred choice for human kinematics [20, p. 69]. These cutoff frequencies were sufficient to capture the high-frequency dynamics in movement, and are consistent with the low 
pass filtering used for muscle activations as found in other studies [22]. The filtered displacement curves were spline fitted using fifth-order piecewise polynomials such that they could be analytically differentiated twice to obtain smooth, continuouslydifferentiable angular velocity and acceleration trajectories for model validation.

\subsection{Electromyography}

To compute muscle activations, sEMG was recorded for seven muscles on the right leg. Electrodes were placed on ideal muscle belly locations following recommendations from the SENIAM project (Surface Electromyography for the Non-invasive Assessment of Muscles) [49]. Electrode placements for the seven muscles of interest are summarized in Table 3.5. Muscles were located using bony landmark references, as shown in Figure 3.2. Some muscles contain multiple muscle groups, whereby a particular muscle was selected as a proxy for the group: gluteus medius for glutei; vastus medialis for vasti; and medial gastrocnemius for gastrocnemii. Prior to electrode placement, the skin was prepared by shaving, lightly sanding [53], and cleaning the area with isopropyl alcohol to remove the surface layer of dead skin cells, thus improving conductivity and reducing motion artifact. Motion artifact corresponds to the electrical noise caused by skin abrasion, electrode movement at the metal/electrolyte interface, and cable movement [54], whereby scratching the skin with a mild abrasive has been shown to be very effective in minimizing its effects [53]. Standard procedure [49] advocates the use of a pair of circular $\mathrm{Ag}-\mathrm{AgCl}$ gel electrodes, $10 \mathrm{~mm}$ in diameter, with an inter-electrode distance (IED) of $20 \mathrm{~mm}$. This ensures sufficient area to gather data from a range of motor units, while reducing cross-talk from neighbouring muscles. It has been shown that the IED influences mean spectral frequency and signal amplitude, whereby large IEDs of around $20 \mathrm{~mm}$ have been used to maximize signal amplitude, while remaining small enough to reduce amplitude variability 
Table 3.5: Electrode placements for the seven major lower-extremity muscles investigated in this study [49].

\begin{tabular}{|l|l|l|}
\hline Muscle & Location & Fibre Orientation \\
\hline $\begin{array}{l}\text { Gluteus } \\
\text { Medius }\end{array}$ & $\begin{array}{l}50 \% \text { between iliac crest \& greater } \\
\text { trochanter }\end{array}$ & $\begin{array}{l}\text { Parallel to adjoining line be- } \\
\text { tween locations }\end{array}$ \\
\hline $\begin{array}{l}\text { Rectus } \\
\text { Femoris }\end{array}$ & $\begin{array}{l}50 \% \text { between anterior superior iliac } \\
\text { spine \& superior part of patella }\end{array}$ & $\begin{array}{l}\text { Parallel to adjoining line be- } \\
\text { tween locations }\end{array}$ \\
\hline $\begin{array}{l}\text { Vastus } \\
\text { Medialis }\end{array}$ & $\begin{array}{l}80 \% \text { between anterior superior iliac } \\
\text { spine \& joint space at anterior bor- } \\
\text { der of medial ligament of knee }\end{array}$ & $\begin{array}{l}\text { Nearly perpendicular to ad- } \\
\text { joining line between loca- } \\
\text { tions }\end{array}$ \\
\hline Biceps Femoris & $\begin{array}{l}50 \% \text { between ischial tuberosity \& } \\
\text { lateral condyle of tibia }\end{array}$ & $\begin{array}{l}\text { Parallel to adjoining line be- } \\
\text { tween locations }\end{array}$ \\
\hline $\begin{array}{l}\text { Gastrocnemius } \\
\text { Medial Head }\end{array}$ & Most prominent bulge of the muscle & Parallel to the shank \\
\hline $\begin{array}{l}\text { Tibialis } \\
\text { Anterior }\end{array}$ & $\begin{array}{l}33 \% \text { between tip of fibula \& medial } \\
\text { malleolus }\end{array}$ & $\begin{array}{l}\text { Parallel to adjoining line be- } \\
\text { tween locations }\end{array}$ \\
\hline Soleus & $\begin{array}{l}66 \% \text { between medial epicondyle of } \\
\text { femur \& medial malleolus }\end{array}$ & $\begin{array}{l}\text { Parallel to adjoining line be- } \\
\text { tween locations }\end{array}$ \\
\hline
\end{tabular}


during dynamic conditions [55]. The electrode pairs were oriented parallel to the muscle fibres, providing a differential signal which was referenced to the body's floating potential using a ground electrode placed at the ankle - a bony surface uncorrupted by muscle activity. An 8-channel Delsys Bagnoli EMG system was used for data

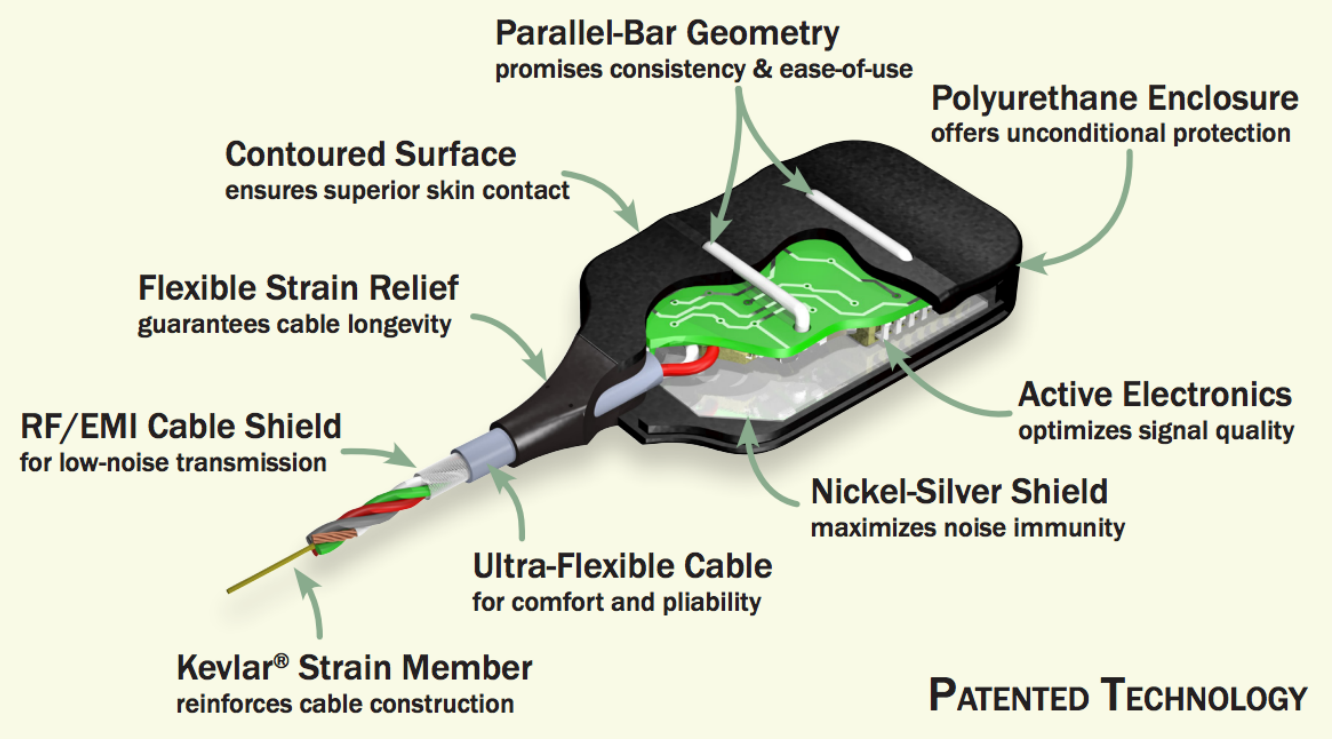

Figure 3.8: Active bar electrodes used with the Delsys Bagnoli EMG system [56.

collection, which uses bar electrodes with a $10 \mathrm{~mm}$ IED, as shown in Figure 3.8. These proprietary electrodes contain active electronics to pre-amplify the signal for improved signal-to-noise ratio characteristics, and employ a unique geometry which has been shown to reduce muscle cross-talk [57]. The sampling frequency was set to $4000 \mathrm{~Hz}$ with a default gain of 1000 to amplify the sEMG signals. A nominal gain of 1000 is suited for typical sEMG signals ranging from $\pm 100 \mu \mathrm{V}$ to $\pm 5 \mathrm{mV}$ resulting in output voltage signals of $\pm 100 \mathrm{mV}$ to $\pm 5 \mathrm{~V}[58]$. The signals were also notch filtered at $60 \mathrm{~Hz}$ prior to acquisition to remove line noise. 


\subsubsection{Post-processing Muscle Activations}

Raw EMG is a bipolar, superimposed signal of multiple motor units firing simultaneously. The signal's amplitude and frequency content are dictated by the intensity of contraction. As the contraction intensifies, more motor units are recruited and their firing rate increases; referred to as spatial and temporal recruitment, respectively $[25$, p. 6, 71]. The recorded signal contains low-frequency noise from motion artifact 22]. As such, the signals were band-pass filtered between 30-450 Hz using a zero-lag fourth-order Butterworth filter, then rectified, normalized to the respective muscle's maximum voluntary contraction, and low-pass filtered at $6 \mathrm{~Hz}$ to acquire the EMG envelope $e(t)$ which resembles the time-varying force-generation profile. As is typical of mechanical motors, muscle exhibits low-pass-filtering characteristics in force generation due to its viscoelasticity and time delays associated with the transmission of action potentials 22 .

Maximum voluntary contractions (MVC) require carefully-planned protocols as simply asking participants to perform an isometric flex is not representative; whereby higher amplitude signals can be seen during dynamic manoeuvres within normal data collection. Alternatively, performing a specific dynamic task has been shown to produce higher MVC peaks and better uniformity across participants [59]. For the experiments, a squat-jump MVC protocol was devised whereby participants were asked to perform ten, maximum-height squat jumps with feet placed shoulder-width apart. It was found that all seven muscles sustained maximum contractions at different phases throughout the repetition. The largest contraction peak for each muscle was individually selected within the ten repetitions performed.

Once the normalized envelope $e(t)$ is obtained, the discretized recursive filter and activation dynamics model, described in Section 2.2, can be used to compute neural excitation $u(t)$ and muscle activation $a(t)$. The post-processing steps for EMG are 
summarized in Figure 3.9 .

\subsection{Energy Expenditure}

An independent measure of metabolic energy expenditure was required to validate the muscle energy expenditure model described in Section 2.4. A common form of measuring energy expenditure is through the use of a metabolic respirometry analyzer [16]. The body's respiratory system serves to provide $\mathrm{O}_{2}$ to the blood while removing $\mathrm{CO}_{2}$ through gas exchange at the capillaries in the lungs' alveoli. In a relaxed state, $12-15$ breaths are taken per minute at a volume of $300-500 \mathrm{~mL}$ each, known as the tidal volume [60]. As the rate of exercise increases, the demand for $\mathrm{O}_{2}$ increases, and with it, the tidal frequency rises to meet this demand. Simultaneously, the body responds by increasing heart rate to facilitate the increased gas exchange at the alveoli. As a result, the $\mathrm{O}_{2}$ consumed and $\mathrm{CO}_{2}$ produced in each breath increases with the rate of respiration.

The Qubit breath-by-breath BBB1LP 60 metabolic analyzer used in this study measures the tidal volume in each breath, as well as the $\mathrm{O}_{2}$ and $\mathrm{CO}_{2}$ concentrations in the inhaled and exhaled air. This determines the instantaneous production of $\mathrm{CO}_{2}$ and consumption of $\mathrm{O}_{2}$. The ratio between these metrics is used to calculate the respiratory exchange ratio $(\mathrm{RER})$ :

$$
\mathrm{RER}=\frac{\mathrm{CO}_{2} \text { production }}{\mathrm{O}_{2} \text { consumption }}
$$

When metabolism is at steady state, the RER is a good estimate of the respiratory quotient (RQ), which indicates the proportions of carbohydrates, fats, and proteins metabolized to obtain chemical energy for the body [60]. For instance, the oxidation 

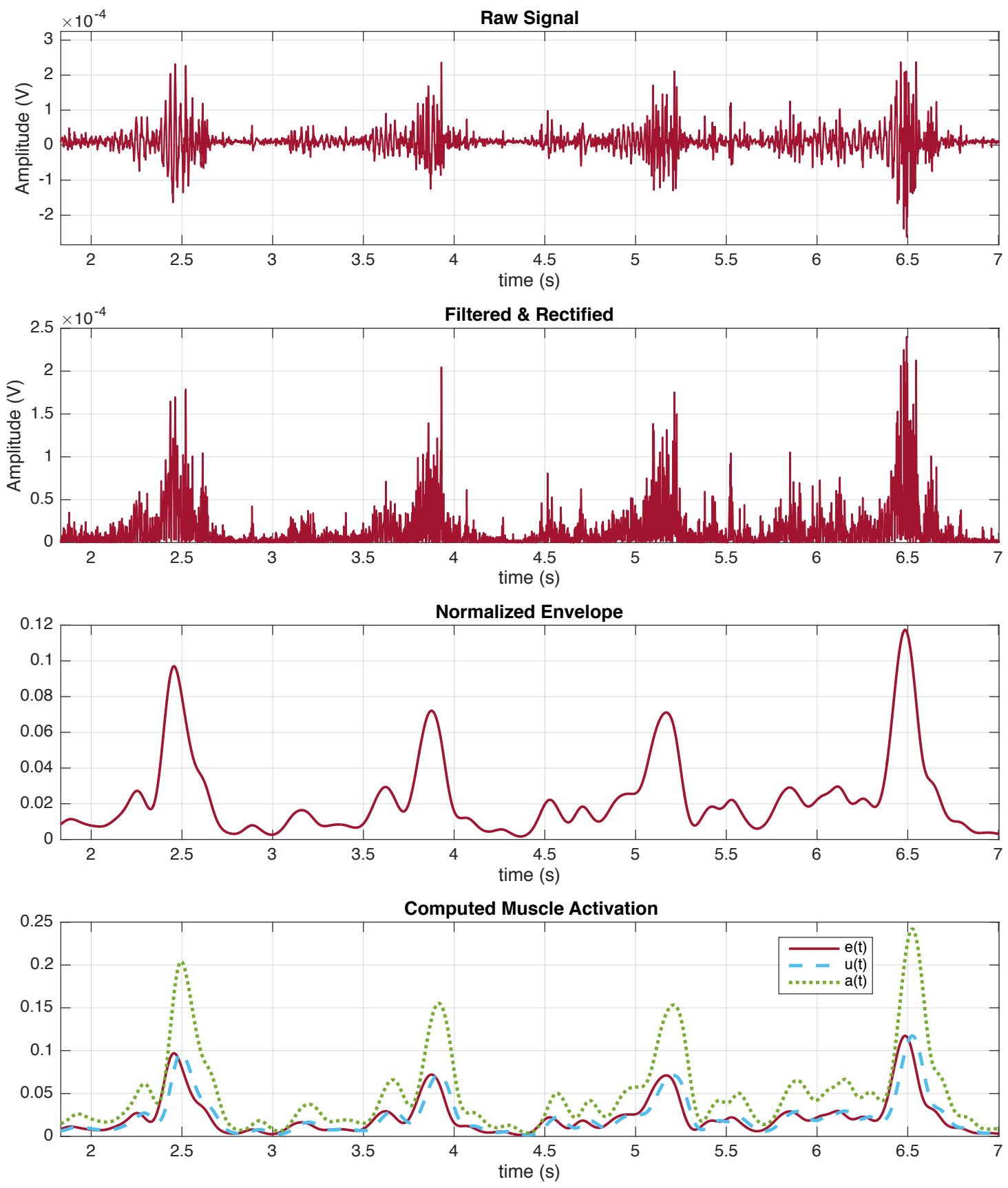

Figure 3.9: Processing of raw EMG signal. 
of glucose, a carbohydrate is given by 60 :

$$
6 \mathrm{O}_{2}+\mathrm{C}_{6} \mathrm{H}_{12} \mathrm{O}_{6} \rightarrow 6 \mathrm{CO}_{2}+6 \mathrm{H}_{2} \mathrm{O}
$$

yielding an RQ of:

$$
\mathrm{RQ}=\frac{6 \mathrm{CO}_{2}}{6 \mathrm{O}_{2}}=1
$$

By comparison, oxidation of palmitic acid, a fatty acid is given by [60]:

$$
23 \mathrm{O}_{2}+\mathrm{C}_{16} \mathrm{H}_{32} \mathrm{O}_{2} \rightarrow 16 \mathrm{CO}_{2}+16 \mathrm{H}_{2} \mathrm{O}
$$

yielding an RQ of:

$$
\mathrm{RQ}=\frac{16 \mathrm{CO}_{2}}{23 \mathrm{O}_{2}}=0.7
$$

The metabolic ratio of these macromolecules used depends on the type of exercise performed and the individual's diet. Typically $40 \%$ of energy is derived from carbohydrates and $60 \%$ from fats during rest, resulting in an RQ of 0.85 . As work increases, energy production shifts to the much faster oxidation of carbohydrates. The RER can exceed 1.0 during intense exercise above the lactate threshold. The lactate threshold represents the exercise intensity at which blood concentration of lactate or lactic acid begins to increase exponentially, which usually occurs at $75 \%$ of maximum oxygen intake. Protein is typically used when carbohydrate reserves are low from starvation or extreme, prolonged exercise 60].

Assuming steady state metabolism, by measuring the RER at each breath, the relative metabolism of fats and carbohydrates can be estimated, which determines the amount of energy produced per litre of $\mathrm{O}_{2}$ as shown in Table 3.6. It is important to 
Table 3.6: Relationship between RER, energy, and food source 60.

\begin{tabular}{llll}
\hline RER & $\begin{array}{l}\text { Energy } \\
\left(\mathrm{kCal} / \mathrm{LO}_{2}\right)\end{array}$ & $\begin{array}{l}\text { \% Energy from } \\
\text { Carbohydrate }\end{array}$ & $\begin{array}{l}\text { \% Energy } \\
\text { from Fat }\end{array}$ \\
\hline 0.70 & 4.69 & 0 & 100 \\
0.75 & 4.74 & 15.6 & 84.4 \\
0.80 & 4.80 & 33.4 & 66.6 \\
0.85 & 4.86 & 50.7 & 49.3 \\
0.90 & 4.92 & 67.5 & 32.5 \\
0.96 & 4.99 & 84.0 & 16.0 \\
1.00 & 5.05 & 100 & 0 \\
\hline
\end{tabular}

note the differences in trained and untrained individuals when analyzing respirometry measurements. Trained subjects generally have a higher stroke volume - more blood is pumped from the ventricles with each beat of the heart; and they ventilate less - lower breathing frequency, at rest. As a result, exhaled air concentrations differ considerably at about $14 \% \mathrm{O}_{2}$ and $18 \% \mathrm{O}_{2}$ for trained and untrained subjects respectively. The reduced breathing frequency due to training means that air remains longer in the lungs, resulting in more efficient gas exchange. Thus untrained individuals work at a higher relative level of exercise during the same absolute level of activity [60]. The metabolic energy consumption measurements used in this study were relative to the resting metabolic rate at the beginning of the experiments, and were collected for an additional $100 \mathrm{~s}$ after each motion profile to allow breathing frequency and $\mathrm{O}_{2}$ and $\mathrm{CO}_{2}$ levels to return to resting levels. As shown in Figure 3.10, participants wore a face mask, which through the pneumotach, measures flow rate as a differential pressure across the pneumotach via the ptach port on the S147 analyzer measuring atmospheric pressure. Inhaled and exhaled air is sub-sampled at a fixed, known flow rate of $300 \mathrm{~mL} / \mathrm{min}$ controlled by the rotameter. Prior to entering the sampling tube, moisture is equilibrated to ambient air via the Nafion tube, and a 25 micron filter is 
used to prevent contamination of the optical gas sensors in the analyzer module. The Lab Pro data acquisition module digitizes the signals for subsequent reduction of data within the LoggerPro software, thereby displaying metabolic energy expenditure and other metrics of interest. Logger Pro performs the necessary volume conversions using the ideal gas law for data collected at ambient and body temperature to standard temperature and pressure $\left(0^{\circ} \mathrm{C}, 101.32 \mathrm{kPa}\right)$. Energy expenditure is then computed in kcal/day using the modified Weir equation [60]:

$$
\dot{E}=3.94 \mathrm{VO}_{2}+1.11 \mathrm{VCO}_{2}
$$

where $\mathrm{VO}_{2}$ and $\mathrm{VCO}_{2}$ represent the volume of $\mathrm{O}_{2}$ consumed and $\mathrm{CO}_{2}$ produced, respectively at standard temperature and pressure conditions. Logger Pro computes the rate of energy expenditure which is then output in kcal/day at $5 \mathrm{~s}$ intervals $(0.2 \mathrm{~Hz})$, which can be converted to $\mathrm{W}$ for comparison with model output.

\subsection{Data Collection Procedure}

Using the instrumentation described in this chapter, the data collection procedure can be summarized as follows.

1. The participant's anthropometric parameters were measured: height, weight, and body segment lengths.

2. On the right leg, skin preparation as outlined in Section 3.5 was performed at SENIAM-recommended [49] muscle belly locations for electrode placements.

3. The participant was asked to conduct ten jump-squats to establish maximum voluntary contraction (MVC) for each muscle.

4. 34 retro-reflective markers were placed on joint centres and other anatomical 


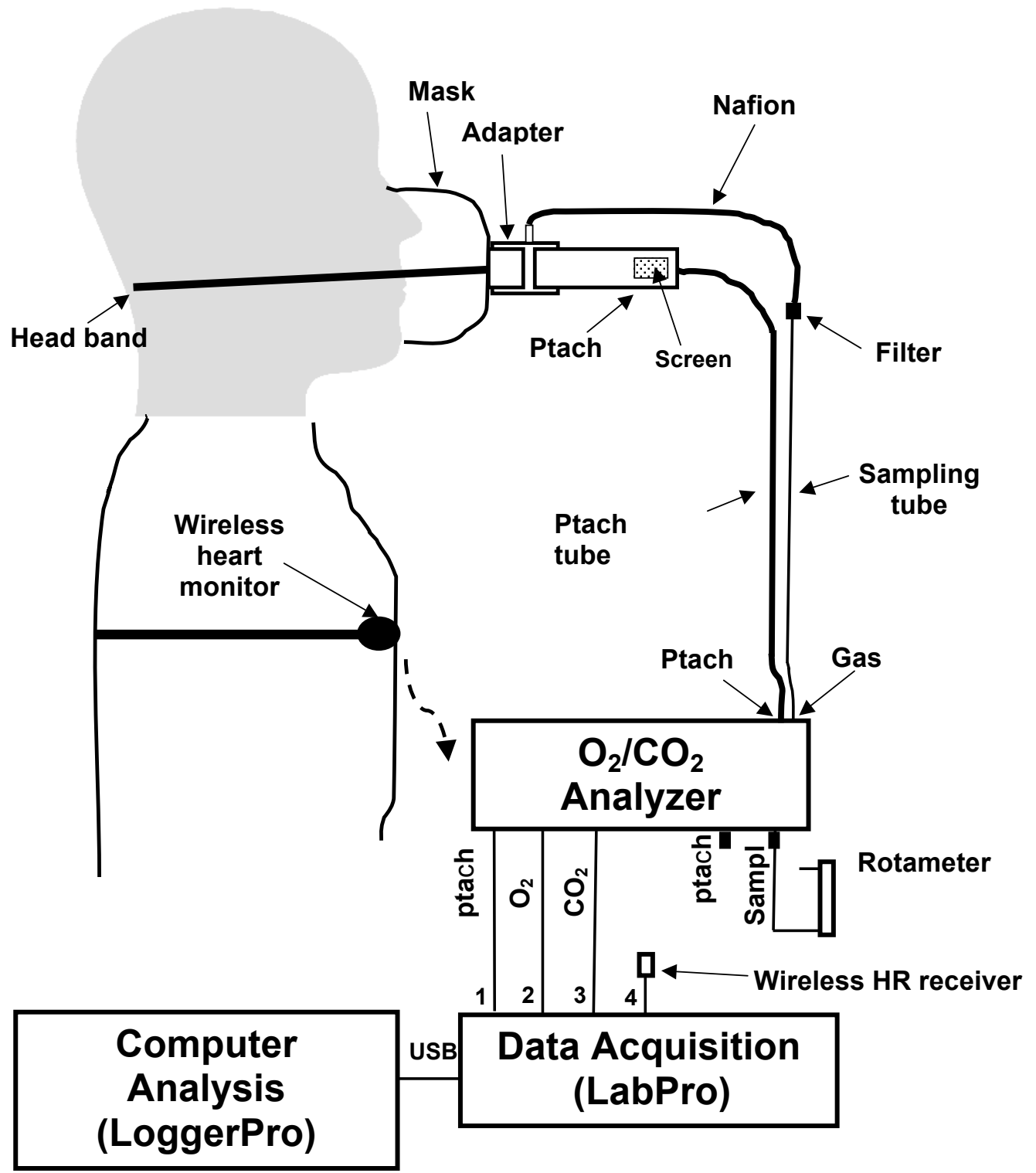

Figure 3.10: Breath by breath metabolic analyzer system setup [60]. 
locations suggested by OptiTrack to define a skeletal model for the subject and to track the motion of limb segments to determine joint rotations.

5. The Qubit Breath-by-Breath BBB1LP $[60]$ respirometry analyzer was configured by having the participant wear a face mask to measure breath flow rate and inhalation and exhalation concentrations of $\mathrm{O}_{2}$ and $\mathrm{CO}_{2}$.

6. The motion profile was deployed to the MOOG platform [51] and the participant was instructed to stomp their right foot and sharply exhale simultaneously as the motion profile was initiated in order to synchronize EMG, respirometry, motion base, and motion capture signals.

7. Step 6 was repeated for a total of four distinct motion profiles: chirp profiles in the pitch, surge, and heave directions individually, as well as a combined 3-DOF profile resembling frigate motion.

The post-processed data was resampled at $100 \mathrm{~Hz}$ and time-synchronized for model validation. By matching the distinct foot stomp signatures in knee angle and muscle activations in the knee-extensors vastus medialis and rectus femoris, joint kinematics and muscle activations could be time-aligned to motion profile data. These raw data signatures are shown in Figure 3.11. Note that the activations have been arbitrarily scaled up to plot alongside knee angle. 


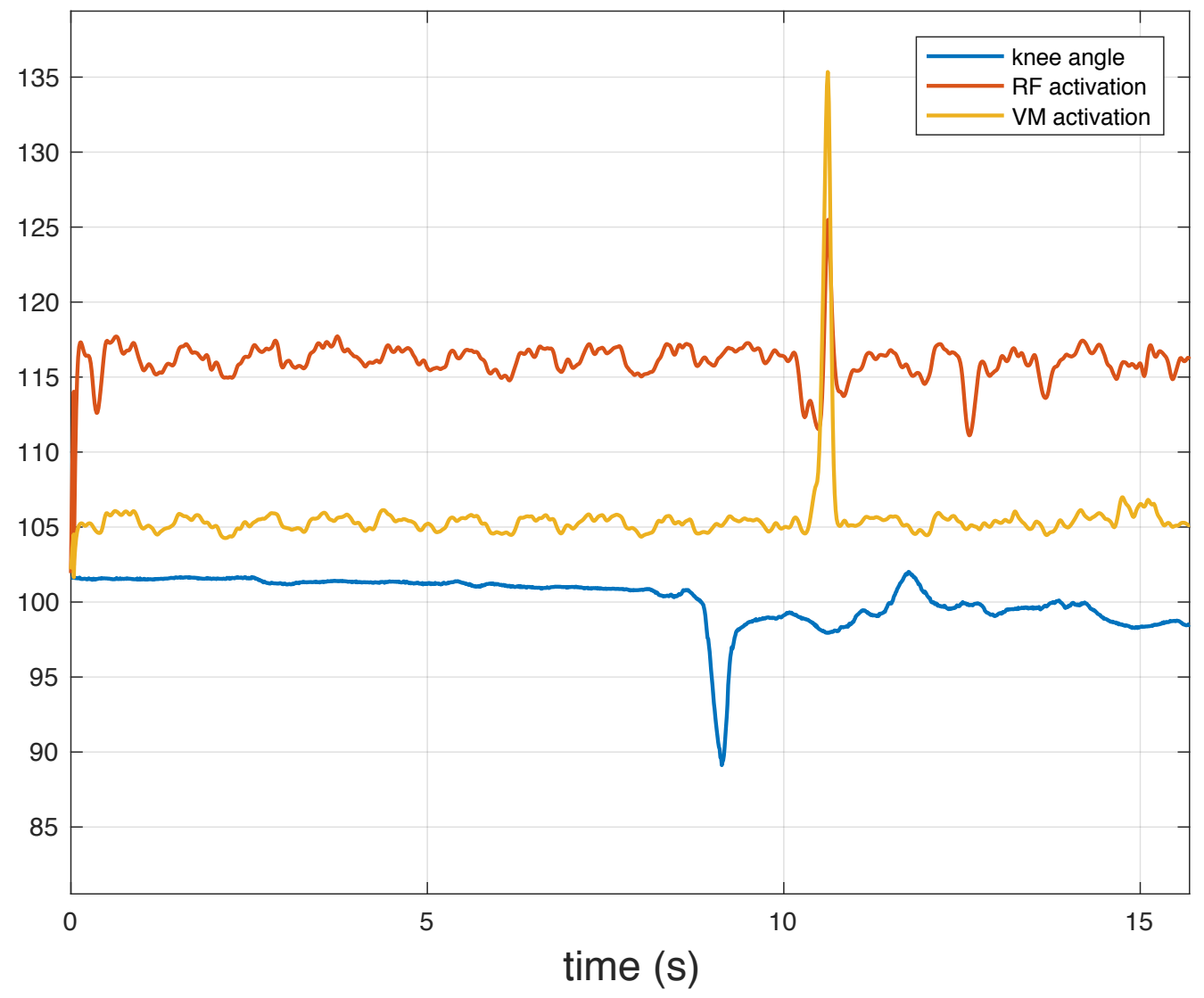

Figure 3.11: Foot stomp signatures in raw data evident from knee angle and knee extensor activations; units are omitted as signals are arbitrarily scaled for collective plotting. 


\section{Chapter 4}

\section{Model Validation}

In order to validate the dynamic modelling framework developed in Chapter 2 , the data collection procedures outlined in Chapter 3 were used to derive model inputs to predict joint torques and energy expenditure to evaluate model output. Referring to Figure 2.1, the model was validated through a comparison of joint torques - through forward dynamics using the musculotendon model (MTM), and through inverse dynamics using the inverted-pendulum skeletal model (3IP). This approach was selected for feasibility as a fully-forward dynamic model used to predict joint kinematics directly would be highly erroneous due to the propagation of errors in simulation. The following sections of this chapter detail the calibration process to tune model parameters to an individual's physiology using a set of training data by verifying the calibrated model with data collected during the various motion profiles, followed by a discussion of the results and their implications.

\subsection{Model Calibration}

Model calibration refers to the process of tuning musculotendon parameters and their inputs to an individual. This is done by first normalizing EMG signals to a maximum voluntary contraction, to quantify muscle activation effort appropriately. Prior to 
data collection, participants were instructed to perform ten maximum height squat jumps to maximally activate all seven muscles throughout the squat cycles. The normalization process of these measurements is detailed in Section 3.5.1.

Second, musculotendon properties require tuning to account for changes in anthropometry and strength to accurately predict musculotendon forces. Given the difficulty in measuring subject-specific parameters, calibration is typically done via optimization on a set of training data. After motion profile data collection, participants were instructed to perform a series of repeated, full-range-of-motion body-weight squats with feet shoulder width apart on the stationary platform. This served as an independent data set for calibration.

\subsubsection{Parameter Tuning}

With the large number of parameters in each portion of the musculotedon modelling process, the risk of "overfitting" the model to a data set is high if too many parameters are tuned. To provide good predictive capability, it is important that strictly physiological parameters are adjusted within reasonably determined bounds to attain a match, such that the tuned model can be applied to novel tasks. The common approach is to vary subject-specific physiological parameters such as optimal muscle fibre length $L_{o}^{M}$, tendon slack length $L_{s}^{T}$, and maximum isometric force $F_{i s o}^{\max }$, as these represent characteristics that are unique to an individual as a function of their anthropometry and physique. For simplicity, the default musculotendon geometrical parameters $\left(L_{o}^{M}, L_{s}^{T}\right)$ derived by Delp [26] were kept constant to maintain consistency with the musculotendon geometry model, which was also unadjusted. To tune the model, only maximum isometric force was varied during a nonlinear least-squares optimization. The parameters were adjusted by applying a scaling factor allowed to

vary within a biologically reported range of $\pm 50 \%$ based on standard deviations of average muscle parameters from numerous investigators [40]. The cost function serves 
to minimize root mean square error between torque predictions over $n$ data points as follows:

$$
\begin{gathered}
J_{j}=R M S E=\sqrt{\frac{1}{n} \sum_{i=1}^{n}\left(T_{i, j}^{3 I P}-T_{i, j}^{M T M}\right)^{2}} \\
\|\mathbf{J}\|=\sqrt{J_{A}^{2}+J_{K}^{2}+J_{H}^{2}}
\end{gathered}
$$

where for a given joint $j$ and time instant $i, T_{i, j}^{M}$ and $T_{i, j}^{3 I P}$ represent the resultant torques derived using the MTM and 3IP models, respectively. This cost function represents the minimization of root mean square error (RMSE), and yields a $J_{j}$ value for each joint. The Euclidean norm $\|\mathbf{J}\|$ is taken as the scalar minimization criteria such that the largest error in torques is penalized over the other two.

Using this optimization process, a match between joint torques was attained by tuning force parameters for each of the seven musculotendon units for the seven participants. Figure 4.1 illustrates the match in joint torques attained through model calibration, and the tuned parameters are tabulated in Table 4.1. While the minimization process is simplified to one parameter, such that the results should only be used as a guideline, they suggest gender differences in thigh musculature of the vasti and glutei as expected. Since no further instruction was provided, participants were found to raise their arms in front of them to maintain balance, and slightly raise their heels, due to inflexibility, at the end of their range of motion. These explain the observed errors, particularly in ankle torque, as they violate the assumptions made for moment of inertia estimation of the HAT segment, and the geometrical constraints between foot and platform for correct force balance. Muscle recruitment patterns and forces are plotted along with corresponding joint angles in Figure 4.2. Agonist and antagonist co-activation for knee extensors and flexors is clearly illustrated in 

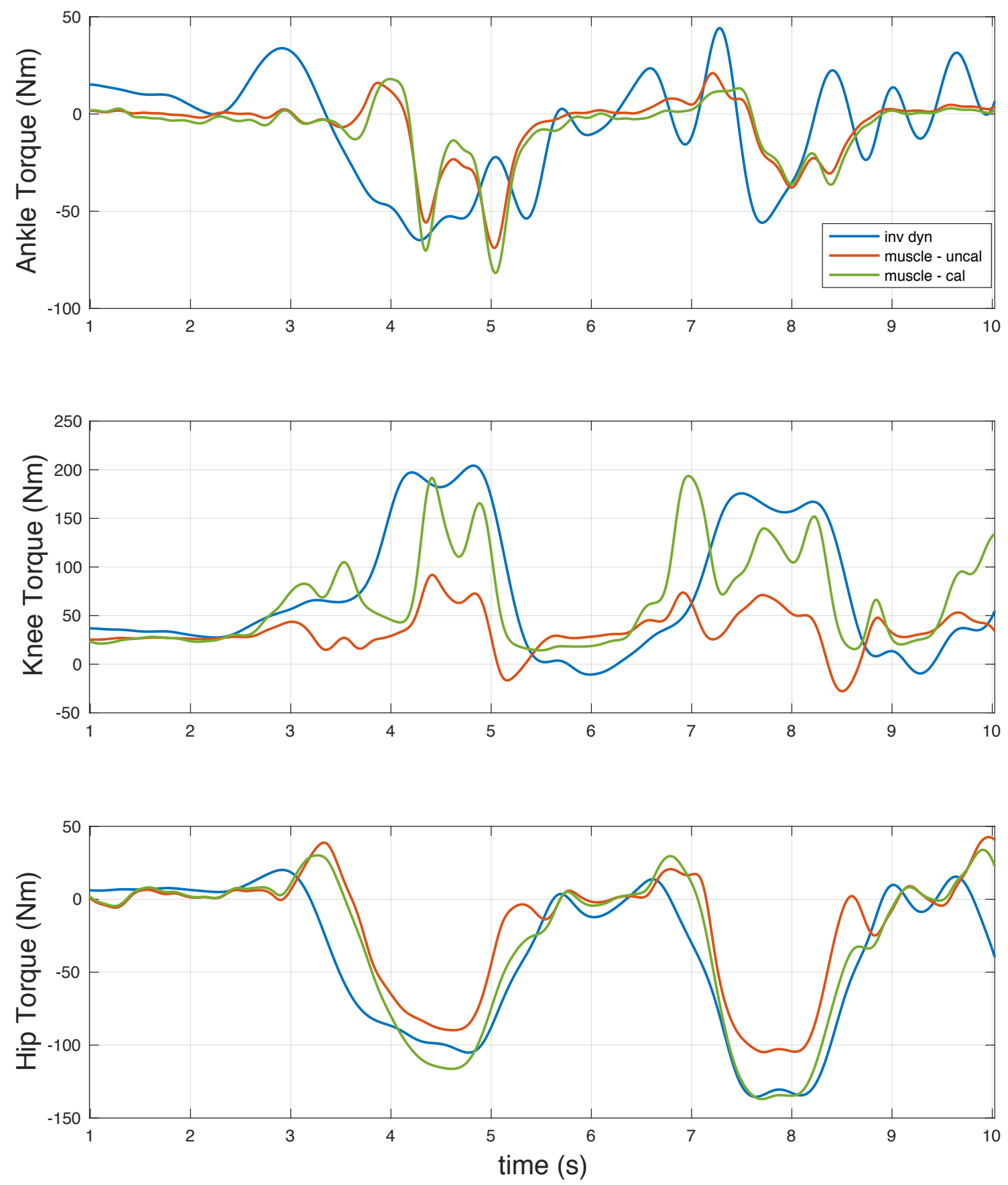

Figure 4.1: Calibrated and uncalibrated joint torques comparison of MTM and 3IP models during body-weight squats. 

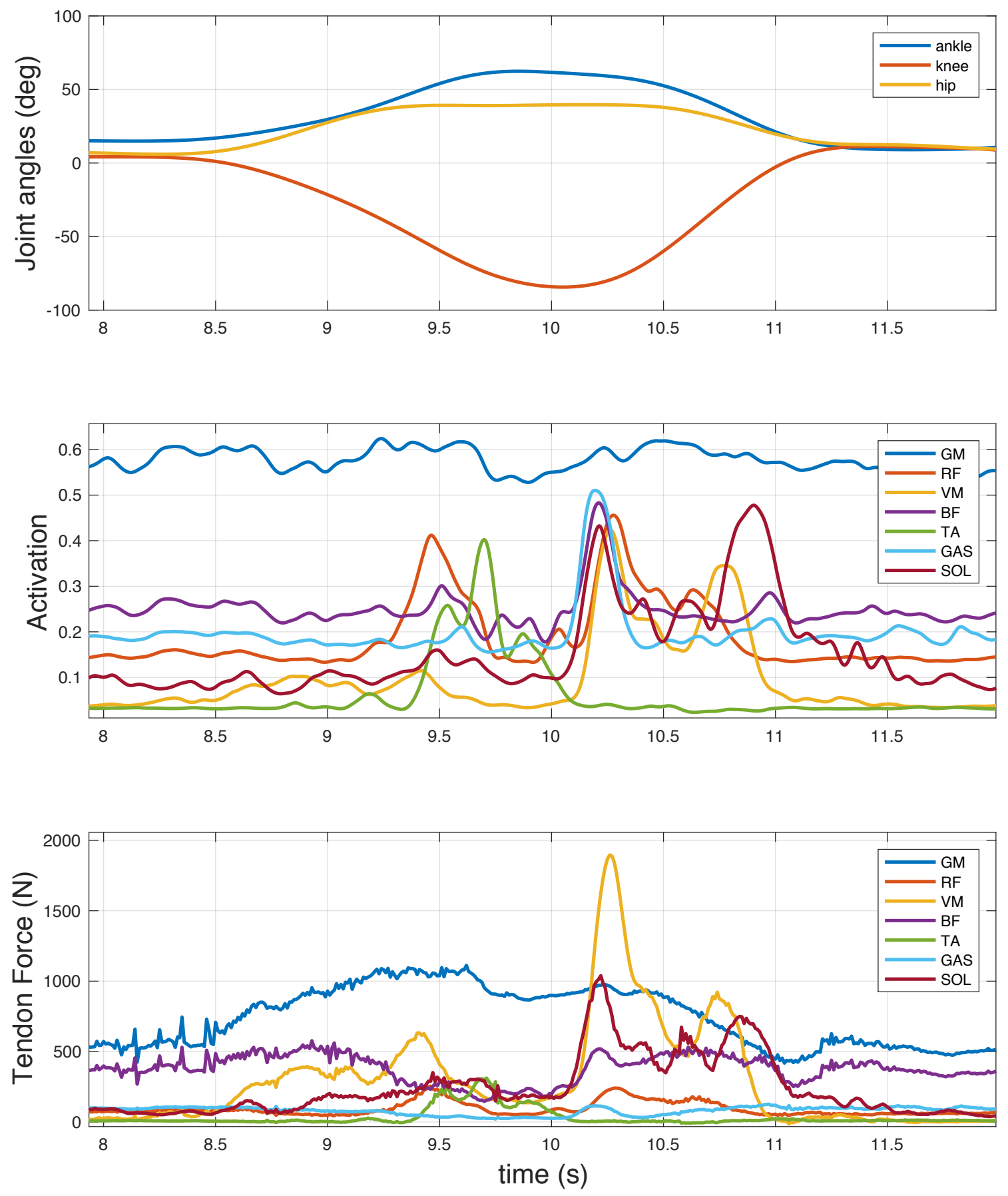

Figure 4.2: Joint kinematics, muscle activations and musculotendon forces during a body-weight squat. 
Table 4.1: Tuned maximum isometric muscle forces $(\mathrm{N})$ for seven participants with nominal values taken from OpenSim 26] representing averages from numerous investigators.

\begin{tabular}{lcccccccc}
\hline Subject & Nom. & 1 & 2 & 3 & 4 & 5 & 6 & 7 \\
\hline Height $(\mathrm{m})$ & - & 1.55 & 1.83 & 1.75 & 1.80 & 1.85 & 1.75 & 1.75 \\
Mass $(\mathrm{kg})$ & - & 46 & 77 & 76 & 102 & 74 & 65 & 75 \\
Sex & - & $\mathrm{F}$ & $\mathrm{M}$ & $\mathrm{M}$ & $\mathrm{M}$ & $\mathrm{M}$ & $\mathrm{F}$ & $\mathrm{M}$ \\
\hline GLU & 1944 & 972 & 1555 & 978 & 974 & 1567 & 1038 & 1179 \\
RF & 1169 & 1224 & 1420 & 936 & 1750 & 1358 & 835 & 1646 \\
VAS & 4530 & 2267 & 6659 & 4133 & 6785 & 6342 & 3530 & 4461 \\
BF & 2594 & 1297 & 1322 & 1302 & 1298 & 1304 & 1315 & 1298 \\
TA & 1759 & 2026 & 2628 & 2646 & 1880 & 1909 & 2550 & 2635 \\
GAS & 2241 & 1122 & 3358 & 3370 & 3362 & 2365 & 3361 & 2988 \\
SOL & 5137 & 2569 & 4814 & 5737 & 7706 & 6153 & 3345 & 2571 \\
\hline
\end{tabular}

activation profiles of the vasti and biceps femoris during both descent and ascent to produce controlled, coordinated motion. During an investigation of muscle recruitment in deep squat cycles [61], vasti (VM), gastrocnemius (GAS), and soleus (SOL) involvement was found to be generally dominant, and these muscles are activated throughout the squat cycle. Dahlkvist [61] reports vasti forces reaching up to $3000 \mathrm{~N}$ during ascent, while soleus and gastrocnemius forces reach 500-600 N. These findings are consistent with those seen in Figure 4.2. Due to heel rise, however, the observed forces suggest a compensation in soleus effort, due to the corresponding reduction in gastrocnemius force, which can be attributed to individual form and the range of motion. 


\subsection{Results}

\subsubsection{Chirp Motion Profiles}

The simulated chirp profiles consist of frequency sweeps from 0.01 to approximately $2 \mathrm{~Hz}$ in the pitch, surge, and heave directions. The objective was to gradually subject participants to the maximum possible acceleration attainable on the MOOG platform while observing changes in coping mechanisms.

\section{Joint Kinematics}

Literature 62 suggests that during low-velocity surface translations, the body behaves as an inverted pendulum by displacing the body's CoM about the ankle while keeping the trunk aligned with the lower extremity. When velocity of surface translations increases, however, hip torques are added to ankle torque to maintain equilibrium.

Subjects portrayed slightly different behaviour during data collection with chirp profiles. Figure 4.3 illustrates the changes in coping mechanisms throughout the frequency sweep of a typical subject. While joint angles suggest a similar trend where ankle displacements increase with surface translation velocity, muscle activations suggest postural stability was easier to maintain at very low and very high velocities, while requiring active effort for mid-range frequencies, 0.7 to $1.5 \mathrm{~Hz}$, corresponding to 60 to $160 \mathrm{~s}$ in Figure 4.3. The increased effort is particularly prominent for hip flexors and extensors: glutei, rectus femoris, and biceps femoris. Joint displacements confirm this transition period with a distinct change in configuration at $60 \mathrm{~s}$ to cope with this. During the mid-frequency period, hip displacements see the largest oscillations, while ankle displacements continue to rise, until finally steadying at $160 \mathrm{~s}$. This behaviour was generally shared across all participants, with varying prominences in peaks. 

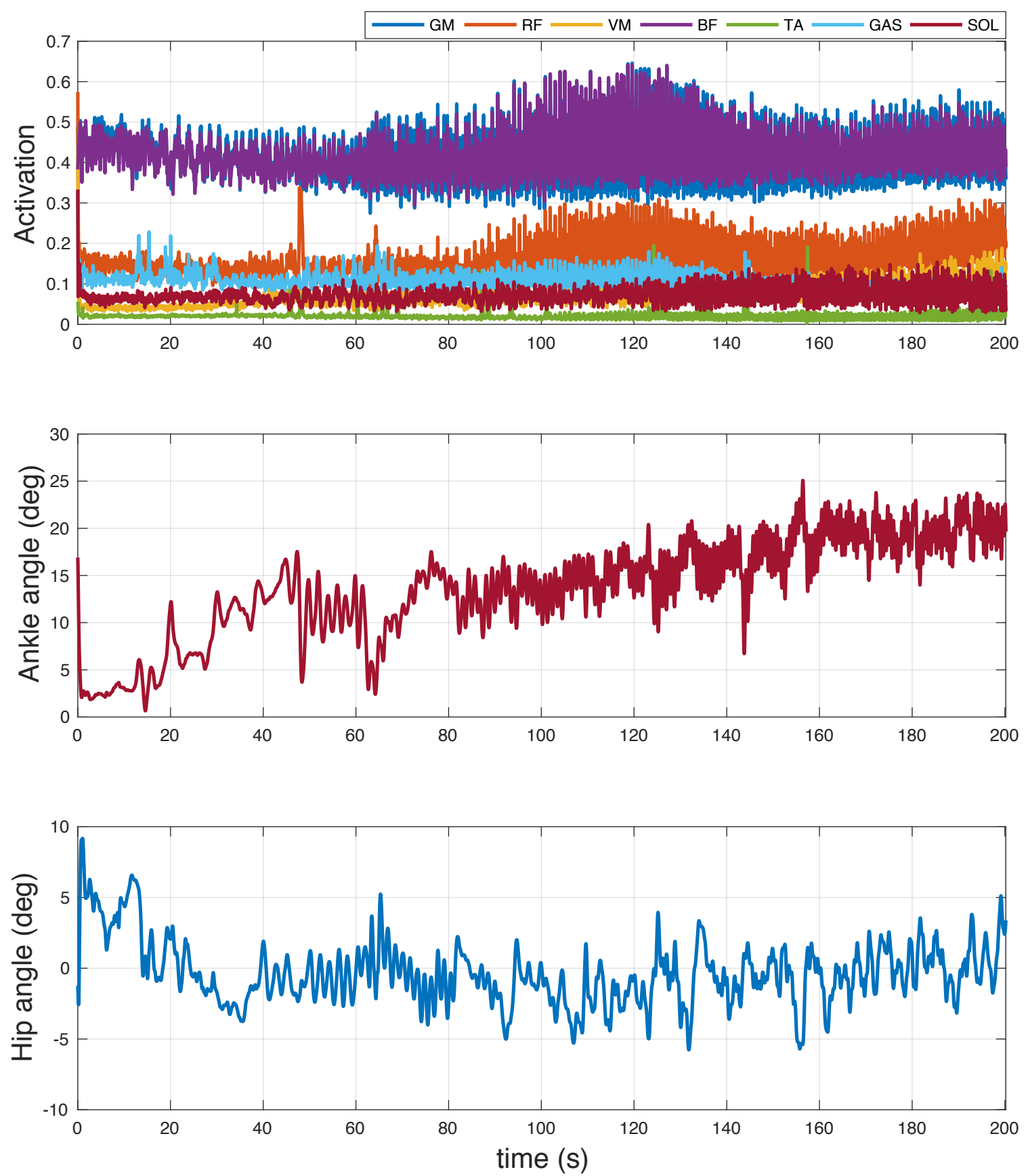

Figure 4.3: Muscle activations and joint kinematics illustrating coping mechanisms during a surge chirp profile. 


\section{Joint Torques}

Figure 4.4 illustrates typical MTM output of joint torques compared to those attained using inverse dynamics with the 3IP model during the surge oscillations. Peaks are tracked well across highly dynamic movement; however, an approximately $500 \mathrm{~ms}$ time delay is observed where the muscle model leads the inverse dynamics output for ankle torques, but lags for hip torques. This suggests a possible delay in force production resulting from a proactive and reactive compensation for the two joints, respectively. For instance, stiffening the joints in anticipation of shank displacement could explain early activations in ankle actuators, whereas hip actuator activation results from a necessity for trunk CoM correction.

\section{Energy Expenditure}

Since energy is both generated and dissipated by the muscles, muscle power output represents a bipolar signal, which is roughly equal in magnitude in the positive and negative directions for the symmetrical chirp motions. Positive work represents work done during a concentric contraction, whereby the muscle fibres shorten to perform work, whereas eccentric contractions result in muscle lengthening which corresponds to negative work being performed. Thus, during the motion profiles generated here, the muscles co-activate to both decelerate the body from the induced motion, and apply positive work to correct posture to balance the CoM. Similarly, for comparison, rate of mechanical work $\dot{W}$ using the 3IP model was computed as follows:

$$
\dot{W}_{i}=\sum_{j=1}^{3} T_{i, j}^{3 I P} \dot{\theta}_{i, j}
$$

where for each time instant $i$, the power output of joint $j$ is computed as the product

of the joint's torque contribution $T_{i}^{3 I P}$ and angular velocity $\dot{\theta}_{i}$. To evaluate energetics 

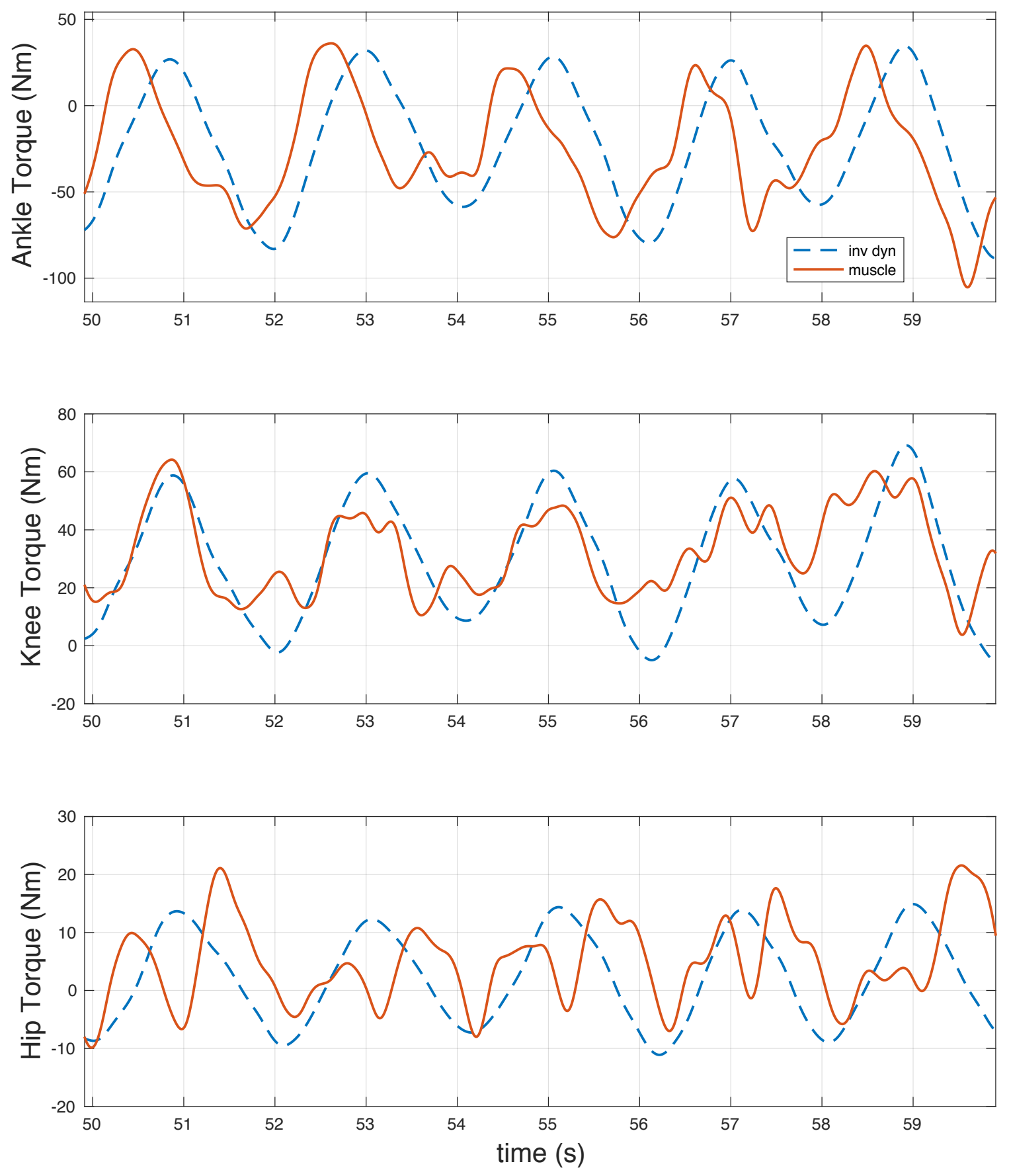

Figure 4.4: Joint torques from MTM muscle model and 3IP inverse dynamics during a surge chirp profile. 
during the chirp profiles, the MTM and 3IP model results of power output were rectified to compare with the total body energy expenditure measured by the BBB1LP metabolic analyzer. It is important to note that the metabolic analyzer measures total energy expenditure which includes a resting metabolic rate, diet-induced thermogenesis, and activity energy expenditure [60]. The combination of resting metabolic rate and diet-induced thermogenesis represent the energy required to maintain basic cellular function and metabolism, while activity energy expenditure alone can be measured from gross muscular activity. A comparison of the energetics is illustrated in Figure 4.5. The motion is stopped at $200 \mathrm{~s}$; however, data collection for respirometry was continued for an additional 100 s to observe a return to the resting metabolic rate. The general increase in the rate of energy expenditure as time progresses is shared by both models and the respirometry data; however, the discrepancy represents an overall increased energetic state of the body during the induced motions which cannot be captured by the task-related power output from seven muscles alone. This increased energetic state may include work done by other muscles such as the core stabilizers, swaying of the arms, and the increase in substrate uptake of carbohydrates, fats, and proteins to meet the increased energy demand.

Nevertheless, the results between MTM and 3IP models are generally consistent given the differences in energetics represented by the two signals. The two models are in agreement during the mid-frequency range during which muscle involvement is greatest, as seen in the activation plot in Figure 4.3. However, during the low- and high-frequency periods, the discrepancy between MTM and 3IP suggests an increased co-activation energy draw that is more accurately represented by the muscle model. These trends indicate that the body comfortably adapts and synchronizes to the induced motion in the mid-frequency range, whereas the effort involved to stiffen the joints in the low- and high-frequency ranges results in reduced angular velocity that underestimates the power requirement involved. 

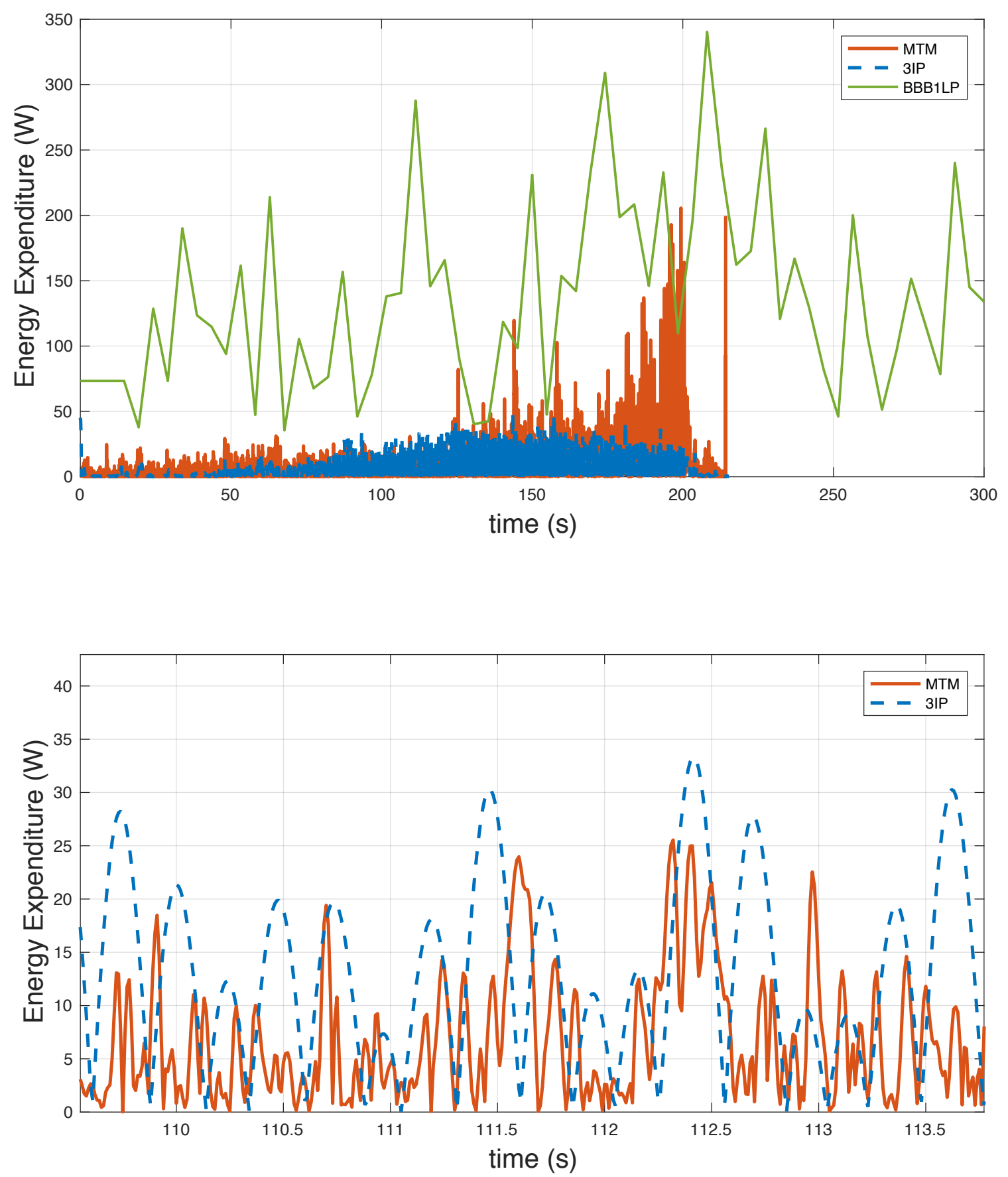

Figure 4.5: MTM and 3IP model energetics compared with total body energy expenditure measured with the BBB1LP analyzer during a surge chirp profile (top); a magnified view detailing the MTM and 3IP results (bottom). 


\subsubsection{DOF Motion Profiles}

Similarly, model output was evaluated for motion profiles containing simultaneous 3DOF motion, representing the stochastic nature of ship motion. The motions were designed such that no sliding or tipping instability is induced, and participants did not deviate foot position to maintain postural stability. However, participants were occasionally found to adopt knee-bent braced positions and made occasional arm movements to maintain stability. This represents a significant cognitive component to maintaining stability whereby kinematics of the motion alone are not good predictors of joint kinematics [13]. It was interesting to note that irrespective of the type of coping mechanism adopted, the head would remain steady while joint configuration adapted to the stochastic motion.

A comparison of joint torque trajectories during the 3DOF motion profile is shown in Figure 4.6, with torque profiles of all seven subjects presented in Appendix B. The resulting RMSE, computed using Equation 4.1, is tabulated for each of the seven subjects in Table 4.2. To evaluate inter-subject variability, RMSE values are also normalized to body mass, yielding values around $0.2 \mathrm{Nm} / \mathrm{kg}$ that are comparable to those obtained in similar studies evaluating muscle-derived torques [40]. In general, model-derived joint torques track the expected trends with occasional overshoots and oscillations. Similarly, a comparison of energetics during the 3DOF motions is illustrated in Figure 4.7. The results indicate power output of the MTM and 3IP models follow the general oscillatory trend seen in the BBB1LP measurements; however, a quantitative comparison is difficult given the coarse nature of the BBB1LP measurements and the short duration of the test. The results suggest that the increased energy rate from co-activation seen in the MTM results, as compared to the 3IP, are sustained throughout the profile given the unpredictable nature of the motion induced. 

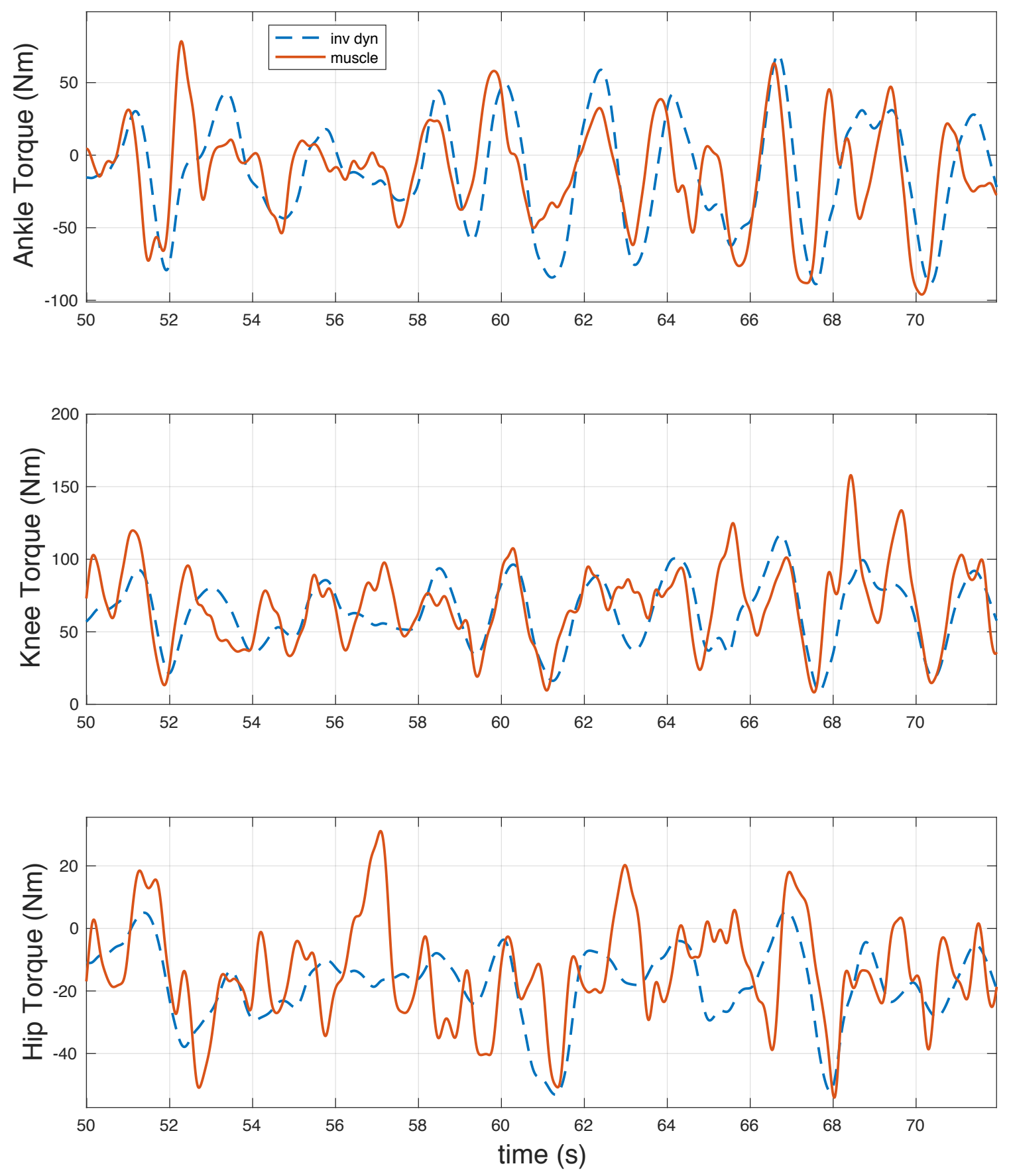

Figure 4.6: Joint torques from MTM muscle model and 3IP inverse dynamics during a combined 3DOF motion profile. 

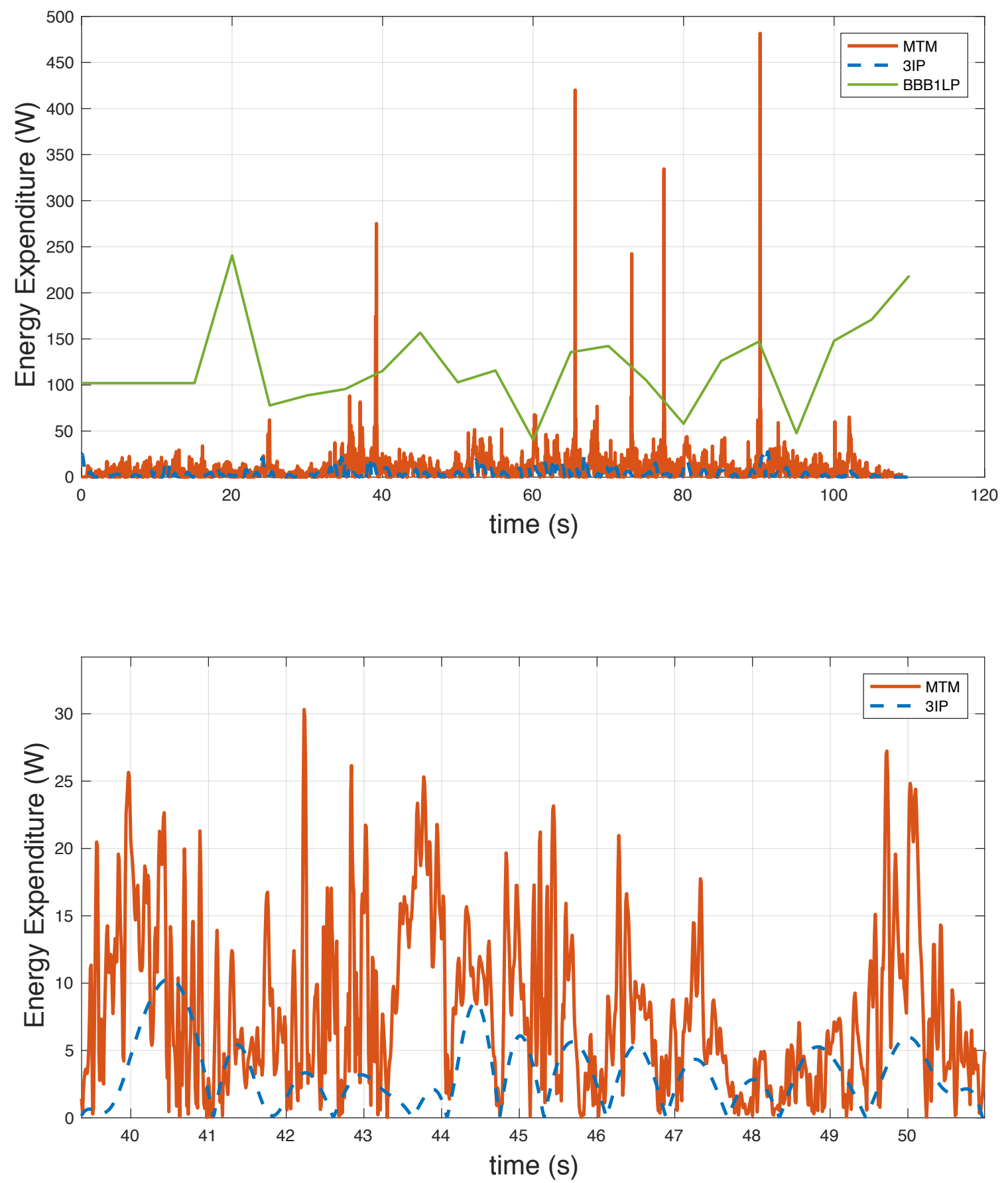

Figure 4.7: MTM and 3IP model energetics compared with total body energy expenditure measured with the BBB1LP analyzer during a combined 3DOF motion profile (top); a magnified view detailing the MTM and 3IP results (bottom). 
Table 4.2: Absolute and normalized (to subject body weight) RMSE of joint torque model predictions during combined 3DOF motion profile representative of frigate deck motion.

\begin{tabular}{ccccccc}
\hline Subject & $T_{A}(\mathrm{Nm})$ & $\bar{T}_{A}\left(\frac{\mathrm{Nm}}{\mathrm{kg}}\right)$ & $T_{K}(\mathrm{Nm})$ & $\bar{T}_{K}\left(\frac{\mathrm{Nm}}{\mathrm{kg}}\right)$ & $T_{H}(\mathrm{Nm})$ & $\bar{T}_{H}\left(\frac{\mathrm{Nm}}{\mathrm{kg}}\right)$ \\
\hline 1 & 11.98 & 0.26 & 8.11 & 0.18 & 6.76 & 0.15 \\
2 & 12.13 & 0.16 & 9.31 & 0.12 & 5.11 & 0.07 \\
3 & 11.96 & 0.16 & 9.98 & 0.13 & 5.89 & 0.08 \\
4 & 18.07 & 0.18 & 16.94 & 0.17 & 15.61 & 0.15 \\
5 & 17.95 & 0.24 & 14.05 & 0.19 & 12.94 & 0.17 \\
6 & 24.44 & 0.38 & 22.67 & 0.35 & 11.42 & 0.18 \\
7 & 31.19 & 0.42 & 23.20 & 0.31 & 10.46 & 0.14 \\
Mean & 18.25 & 0.26 & 14.89 & 0.21 & 9.74 & 0.13 \\
\hline
\end{tabular}

\subsection{Discussion}

\subsubsection{Modelling Assumptions}

A discussion of the assumptions is crucial to appreciating the quality of the match attained in the comparisons between joint kinetics during coordinated and highly dynamic movements. The anthropometric model used here was intentionally algebraically simple, using just body weight and height as input. However, in doing so, many physiological parameters such as body fat, body mass index (BMI), and bone density, which have a significant influence over inertial properties of limb segments, are neglected, which can greatly affect resulting torques from observed movement. Furthermore, the skeletal dynamic model derived assumes fixed joint centres. While this is a reasonable assumption for hip and ankle joints, it does not account for the complex kinematics of the knee joint, as shown in Figure 2.5, where both muscle moment arms and musculotendon geometry change nonlinearly with knee angle. Although the musculoskeletal geometry model accounts for these nonlinearities, it 
relies on an accurate measurement of joint angles from the motion capture system for musculotendon kinematics.

In using an untuned musculoskeletal geometry model, an implicit assumption is made whereby peak force production with joint kinematics remains constant. The musculoskeletal geometry model uses Delp's model [26] as a basis; however, these values will change with anthropometry and therefore are subject-specific, changing not just joint angles at which maximum force production occurs, but also the nonlinear relationship between moment arms which can result in vastly different torque profiles. Unlike detailed musculoskeletal dynamic models targeted towards the study of precise gait patterns and the effects of rehabilitation, the objective of the model developed here, however, is to provide a framework that is viable for operational planning and to mitigate the risk of injury using a generic model. As a result, the computational savings in the validation process are significant for the small loss in accuracy in doing so.

Similarly, by simplifying the model tuning process by neglecting the variations in tendon slack length and optimal muscle fibre length between participants, maximum force production is affected which will vary the results obtained from the optimization of maximum isometric force alone. It is, therefore, important to note that the tuned force parameters attained here should only be used as a guideline.

\subsubsection{Validation Techniques}

Validation of the model is best done through a comparison of musculotendon forces directly, however, since practical limitations inhibit in vivo measurements of muscle forces, the model is instead calibrated and validated with inverse dynamics estimates

of joint torques. The use of this approach relies on the accuracy of joint kinematics from motion capture. This generally introduces a number of inherent errors.

The relative displacement of markers with respect to the body is generally one of 
the largest sources of error for motion capture. During data collection, the spandex suit tends to crumple, which can have a significant impact on joint angles as it contains the hip and neck markers. Although lower leg markers were attached to the skin, they are susceptible to relative movement with respect to the bone for correct joint centre marking. The joint angles seen during motion profiles were typically under $20^{\circ}$, representing a poor signal to noise ratio. It is important to note that even small errors in joint angles can be responsible for large deviations in joint torques. Intersubject variability of physiological parameters, such as body fat, make it difficult to speculate the size of these errors.

\subsubsection{Calibration and Parameter Tuning}

A high degree of variability is introduced during EMG normalization through the use of MVC protocols. The squat jumps were used in this study to reduce this variability with the use of a dynamic task-based protocol as suggested by similar studies [59] rather than voluntary-flexion based. However, the nature of the protocol is subject to participant interpretation. Participants were instructed to perform ten, maximum height squat jumps. In some cases, individual fitness level and electrode cable tethering invoked reservations in exercising maximal effort during the jumps.

Calibration is generally a balance between physiological correctness and computational efficiency whereby varying additional parameters such as tendon slack length and optimal muscle fibre length can be used to improve model accuracy. The importance of these changes should not be underestimated. For instance, the surgical procedure of moving the insertion point for the rectus femoris to the tendon of the sartorius muscle can have tremendous effects on knee flexion/extension in order to correct stiff-legged gait [26]. To improve subject-specific tuning, it is recommended 
that future implementations include a set of comprehensive calibration trials with dynamometer measurements as a gold standard, and an investigation to make anatomical measurements to better estimate muscle parameters. For instance, musculotendon geometries can be measured through bone insertion and origin lengths, and maximum isometric force can be estimated through limb circumference at selected locations. Alternatively, some studies have suggested the use of scaling factors based on anthropometric data to provide estimates and optimization search spaces for musculotendon geometries [40]. Doing so has a number of advantages. Each joint can be calibrated individually, thereby reducing the optimization search space to tune force parameters. Second, a load cell has the added benefit of capturing high-frequency dynamic components without the noise generated from the differentiation of kinematic data for inverse dynamics computations.

\subsubsection{Rigid-tendon Simplification}

The rigid tendon simplification allows for forward-dynamic computation of muscle forces and torques in under $10 \mathrm{~s}$ (on a $2.3 \mathrm{GHz}$ Intel i7 processor) for a $200 \mathrm{~s}$ trial containing seven muscles, without significant loss in model accuracy [42]. The absolute and normalized RMSEs for torque predictions are similar in magnitude to those obtained from studies using the compliant tendon model for actuators of the knee joint [40]. Without this simplification, solving for these metrics would increase complexity by introducing a first-order differential equation for each muscle, in addition to the two first-order differential equations for each DOF in the rigid-body skeletal dynamic model. Similar models for gait studies have reported 5-14 hour computation times for a fully-forward dynamic model simulation [19]. For prediction in planning, this type of computationally-efficient modelling framework is sufficient in providing joint kinetics while still providing insight into musculotendon kinetics for fatigue and injury metrics. 


\subsubsection{Implications of the MTM Model}

Using a neuromuscular model to predict human-body kinetics has several advantages. The model contains constraints represented by the anatomy and physiology of the human body such as isometric musculotendon forces that are consistent with those observed in large populations from in vivo measurements in literature, as well as the complex geometry measurements made to model musculotendon wrapping and the moment arm relationships with joint angle changes. Second, the calibration protocols used allow for a realistic representation of the passive stiffness generated from musculotendon stretching as well as its active force generation. Representing this as a net torsional stiffness is not possible without modelling the mathematics involved at the individual musculotendon level. However, it is important to include all primary torque-producing muscles through a possible simulation of activations from neighbouring, similar-tasked muscles to ensure a viable prediction of passive and active effort through a better estimation of muscle forces [40].

Similarly, the differences in the rate of energy expenditure between the MTM and 3IP models suggest improved accuracy in the task-related energy rate predicted by the MTM model by including the effects of muscle co-activation. By quantifying power output peaks from physiological force generation profiles rather than joint kinematics, the muscle model has potential to provide improved resolution in the analysis of range-of-motion-based task efficiency and risk-of-injury criteria.

\subsection{Summary}

In this chapter, it was shown that the MTM model could be calibrated to physiological characteristics for an individual using a set of training data for generalized predictive capability for other tasks. While model accuracy is largely dependent on accurate anthropometric data and reliable joint kinematics, the model produced good 
agreement with joint torques derived from inverse dynamics, despite the potential for errors. Furthermore, a comparison of energy expenditure represents a plausible estimation of power output from muscle energetics with the limitations of representing only a subset from the seven muscles captured here. Overall, the model indicates good predictive capability with computational efficiency for musculotendon kinetics and energetics in a variety of motion environments. 


\section{Chapter 5}

\section{Conclusions and Future Work}

The harsh environments onboard HSC subject occupants to repeated, high-impact shock loading, forcing them to adopt a semi-squatted posture to attenuate impact with their legs in the interest of comfort and practicality. In doing so, these occupants suffer a host of acute and chronic musculoskeletal injuries that warrants a thorough understanding of human movement in these dangerous motion environments. This work addresses the need to characterize the involvement of active muscle engagement in maintaining postural stability, for the implications in injury prevention through prediction of muscle forces, joint torques, and energy expenditure. This chapter summarizes the developments in each of the preceding chapters by highlighting the contributions of this work, followed by recommendations for future work.

\subsection{Model Development}

Modern modelling techniques for the dynamics of muscle activation and contraction were investigated to refine the process for the development of a computationallyefficient framework for modelling multi-body dynamic models actuated by the major force-generating muscles in the lower extremity. A comprehensive musculoskeletal 
dynamic modelling framework was developed encompassing the geometry of musculotendon kinematics as well as the rigid-body skeletal dynamics for an inverted pendulum model to mimic sagittal-plane movement of the standing individual as a result of motions in 3DOF: pitch, surge, and heave. The developed model utilizes muscle activations and joint kinematics to estimate force production in the seven major musculotendon units in the lower extremity to predict the torques responsible for actuating limb segments about the ankle, knee, and hip joints. The framework is modular in that additional musculotendon units can be easily included by specifying a few physiological parameters.

\subsection{Experiment Design and Instrumentation}

To validate the modelling framework developed, experimental trials with seven participants were conducted by measuring participants' muscle activity, limb movement, and respiration metrics involved in maintaining postural stability during a series of motion profiles in 3DOF. Muscle activations were derived from sEMG measurements of the seven musculotendon units modelled, while joint kinematics were extracted from a motion capture system tracking positional trajectories of strategically-placed markers on limb segments and joint centres. The respirometry system allowed an independent measure of metabolic energy expenditure through volumetric flow rate of inhaled and expired air as well as the respective concentrations of $\mathrm{O}_{2}$ and $\mathrm{CO}_{2}$ in these air flows. A structured data collection procedure was developed to synchronize the measurements and for systematic, subject-specific calibration of muscle activations through a task-based maximum voluntary contraction protocol, as well as training data to calibrate physiological parameters for each of the musculotendon units. 


\subsection{Model Validation}

The modelling framework was calibrated by tuning generic muscle model parameters of a given individual, through an optimization routine to minimize the error in joint torques over a set of training data. The calibrated model allows for generalized predictive capability for musculoskeletal kinetics during generalized tasks, tested through a series of motion profiles exciting the musculoskeletal system in the sagittal-plane. Despite the potential measurement errors in joint kinematics and anthropometric assumptions, the model shows good agreement in joint torque profiles during highlydynamic movements when compared to those derived using inverse dynamics.

\subsection{Future Work}

Due to the stringent requirements of instrumentation and ethical concerns with acquiring experimental data with human participants onboard HSC, it is difficult to validate the dynamic modelling framework developed here with HSC data. The OptiTrack motion capture system requires a sufficient capture volume, a dark environment which is easily contaminated by reflective surfaces, and a specific configuration of marker placement to measure joint kinematics. Recent advancements in motion capture technology have allowed for contrast-based tracking of limb segments 63 that do not require infrared cameras, as well as markerless capture systems 64 that extract and fit silhouettes to a 3D model for the computation of joint kinematics. In addition to simplicity of setup, these systems offer numerous advantages in that they are not vulnerable to artifacts caused by relative movement of the markers with respect to the skin, and do not require a motion-capture suit which is impractical for HSC occupants already carrying heavy equipment. Recent improvements in wireless EMG systems [65] also offer the advantage of tetherless data capture of muscle 
activations without large cables to the amplifier and uninhibited movement for the participant. The following sections suggest improvements to the modelling framework with respect to data collection and model validation with HSC data.

\subsubsection{Optimal Controller}

Optimization techniques offer improved predictive capabilities with the existing modelling framework in a number of ways. In the absence of measured muscle activations, the optimization techniques described in Section 1.2.4 allow for a physiologicallycompliant solution for muscle redundancy to compute musculotendon forces via inverse dynamics through measured joint kinematics.

Numerous studies $14,18,23$ have used optimal control to mimic the CNS in order to generate joint torques to simulate human movement with multi-body models. Through parameter estimation with a comprehensive HSC data set with the current modelling framework, commanded neural excitation is treated as the optimization variable providing biofidelic estimations of musculotendon forces to initiate movement and model joint stiffening. This is ideal for predictive capabilities where no data is given, allowing for injury and risk management in foreseeable wave and boat deck conditions.

\subsubsection{Muscle Fatigue}

The modelling framework developed here is vulnerable to errors in force generation during heavy muscle fatigue. Recent work 66, 67 has introduced a three-compartment model, as shown in Figure 5.1, that describes muscle fibres as being activated $M_{A}$ (actively generating force), fatigued $M_{F}$ (incapable of generating force), or resting $M_{R}$ (recovering after being fatigued), which better represents the state of muscle fibres during sustained contractions. The transition of muscle fibre proportions be- 


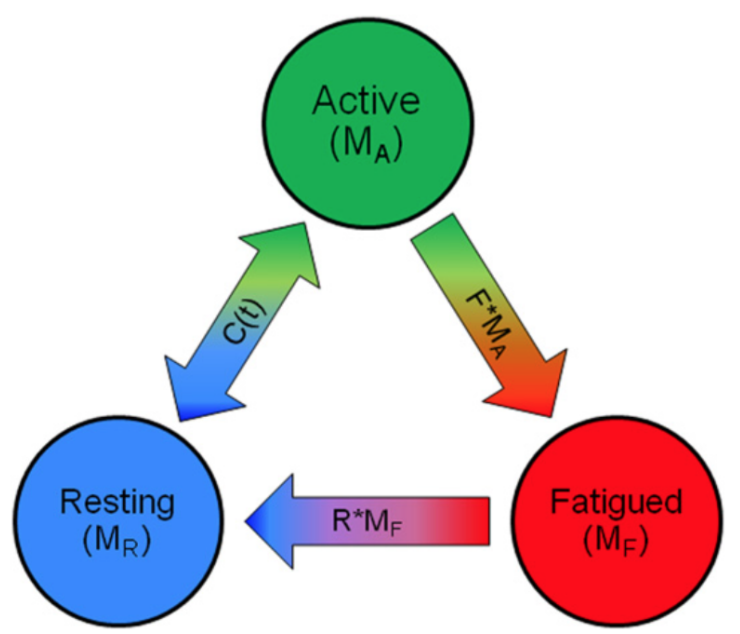

Figure 5.1: Schematic of the three-compartment model illustrating transition of muscle fibre states [67].

tween these states is governed by a muscle activation-deactivation drive $C(t)$, and fatigue and resting rates $F$ and $R$ respectively [66]. While current work is focused on generalizing the model for individual musculotendon units as a function of fibre composition, these parameters tend to remain task-specific for sustained isometric contractions, making application to other tasks with intermittent rest intervals or dynamic conditions difficult [67]. However, the addition of this type of approach to the existing framework has the potential to produce an altered force generation curve such that activation, rather than remaining linear, can mimic the recruitment of slowor fast-twitch fibres depending on the level of task intensity.

\subsubsection{Model Fidelity}

The rigid-body dynamic model developed adopts a number of simplifying assumptions that were suited specifically to sagittal-plane motions in the semi-squatted posture adopted by HSC occupants. To improve fidelity to encompass a larger range of movements, several improvements are suggested. Range of motion in the hip and ankle joints can be increased to 3DOF, namely hip rotation (transverse plane), 
hip adduction-abduction (frontal plane), ankle rotation (transverse plane), and ankle pronation-supination (frontal plane). In doing so, the body's response to complete 6DOF motion profiles can be modelled to include effects of roll, yaw, and sway motions. This allows the consideration of lateral disturbances, particularly movement as a result of roll motion, which is a common source of motion-induced interruptions on the boat deck. This requires modelling additional musculotendon units, which was limited by the number of EMG channels with the amplifier available for experimentation in this research. To properly consider muscular involvement in these additional DOF, the muscle geometry model is easily modified to accommodate 3DOF rotations at the hip and ankle, as well as modelling additional musculotendon units that have significant torque contributions to these joint rotations. The nature of this modelling framework allows additional musculotendon units to be defined with a handful of physiological parameters that are widely available in literature.

\subsubsection{Summary}

Overall, the modelling framework developed provides general applicability to postural stability in a wide range of motion environments and lends itself for improvements with respect to muscle fibre fatigue and increased range of motion for future investigation of injury criteria and occupant-seat interaction on high-speed craft. 


\section{References}

[1] S. D. Myers et al. Physiological consequences of military high-speed boat transits. European Journal of Applied Physiology, 111(9):2041-2049, 2011.

[2] ABCD Group. High Speed Craft Human Factors Engineering and Design Guide, ABCD-TR-08-01 V1.0. http://www.20knots-plus.com/book-downloadrefs/, 2008.

[3] W. Ensign et al. A survey of self-reported injuries among special boat operators. Technical report, DTIC Document, 2000.

[4] J. L. Colwell et al. Shock mitigation seat test and evaluation. The Royal Institution of Naval Architects, 2011.

[5] ISO. Mechanical Vibration and Shock: Evaluation of Human Exposure to Wholebody Vibration. Part 1, General Requirements: International Standard ISO 26311: 1997 (E). ISO, 1997.

[6] M. R. Riley et al. Ride severity index-a new approach to quantifying the comparison of acceleration responses of high-speed craft. In Proceedings of the 11th International Conference on Fast Sea Transportation, Hawaii, USA, 2011.

[7] R. Peterson et al. Shock mitigation for the human on high speed craft: development of an impact injury design rule. Technical report, DTIC Document, 2004.

[8] E. L. Stech and P. R. Payne. Dynamic models of the human body. Technical report, DTIC Document, 1969.

[9] ISO. Mechanical Vibration and Shock: Evaluation of Human Exposure to Wholebody Vibration. Part 5, Method for Evaluation of Vibration Containing Multiple Shocks: International Standard ISO 2631-5: 2004 (E). ISO, 2004. 
[10] TASS International. MADYMO. https://www.tassinternational.com/ madymo. Accessed: 2016-12-05.

[11] C. R. Bass. Injury criteria and numerical modeling for high speed craft. 2005.

[12] Zuneid Alam. Implementation of a drop test impact rig for dynamic testing of high speed craft shock mitigation seats and extraction of modal parameters. Master's thesis, Carleton University Ottawa, 2013.

[13] R. G. Langlois et al. Modelling sea trial motion induced interruption data using an inverted pendulum articulated postural stability model. International Journal of Maritime Engineering, 151:1-10, 2009.

[14] Hemami H. and V. C. Jaswa. On a three-link model of the dynamics of standing up and sitting down. Systems, Man and Cybernetics, IEEE Transactions on, 8(2):115-120, 1978.

[15] S. H. Koozekanani et al. On the role of dynamic models in quantitative posturography. Biomedical Engineering, IEEE Transactions on, (10):605-609, 1980.

[16] G. Kaur. Mechanical energy expenditure while maintaining postural stability in shipboard motion environments. Master's thesis, Carleton University Ottawa, 2013.

[17] N. Bourgeois. Prediction of Human Postural Response in Shipboard Environments using Multibody Dynamics and Sensory-based Control. PhD thesis, Carleton University Ottawa, 2015.

[18] A. D. Kuo. An optimal control model for analyzing human postural balance. IEEE Transactions on Biomedical Engineering, 42(1):87-101, 1995.

[19] M. S. Shourijeh and J. McPhee. Forward dynamic optimization of human gait simulations: a global parameterization approach. Journal of Computational and Nonlinear Dynamics, 9(3):031018, 2014.

[20] D. A. Winter. Biomechanics and motor control of human movement. John Wiley \& Sons, 2009

[21] A. Nagano and K. G. M. Gerritsen. Effects of neuromuscular strength training on vertical jumping performance-a computer simulation study. Journal of Applied Biomechanics, 17(2):113-128, 2001. 
[22] T. S. Buchanan et al. Neuromusculoskeletal modeling: estimation of muscle forces and joint moments and movements from measurements of neural command. Journal of Applied Biomechanics, 20(4):367, 2004.

[23] M. S. Shourijeh. Optimal Control and Multibody Dynamic Modelling of Human Musculoskeletal Systems. PhD thesis, University of Waterloo, 2013.

[24] D. Lee et al. A survey of modeling and simulation of skeletal muscle. $A C M$ Transactions on Graphics, 28(4):1-13, 2010.

[25] R. Merletti and P. A Parker. Electromyography: Physiology, Engineering, and Non-invasive Applications, volume 11. John Wiley \& Sons, 2004.

[26] S. L. Delp et al. An interactive graphics-based model of the lower extremity to study orthopaedic surgical procedures. Biomedical Engineering, IEEE Transactions on, 37(8):757-767, 1990.

[27] C. R. Ethier and C. A. Simmons. Introductory Biomechanics: From Cells to Organisms. Cambridge University Press, 2007.

[28] A. F. Huxley. Muscle structure and theories of contraction. Prog. Biophys. Biophys. Chem, 7:255-318, 1957.

[29] A. F. Huxley and R. M. Simmons. Proposed mechanism of force generation in striated muscle. Nature, 233(5321):533-538, 1971.

[30] G. I. Zahalak. A distribution-moment approximation for kinetic theories of muscular contraction. Mathematical Biosciences, 55(1-2):89-114, 1981.

[31] H. S. Gasser and A. V. Hill. The dynamics of muscular contraction. Proceedings of the Royal Society of London. Series B, containing papers of a biological character, 96(678):398-437, 1924.

[32] A. V. Hill. The heat of shortening and the dynamic constants of muscle. Proceedings of the Royal Society of London B: Biological Sciences, 126(843):136-195, 1938.

[33] F. E. Zajac. Muscle and tendon: properties, models, scaling, and application to biomechanics and motor control. Critical Reviews in Biomedical Engineering, 17(4):359-411, 1988.

[34] R. D. Crowninshield and R. A. Brand. A physiologically based criterion of muscle force prediction in locomotion. Journal of Biomechanics, 14(11):793-801, 1981. 
[35] F. C. Anderson and M. G. Pandy. Static and dynamic optimization solutions for gait are practically equivalent. Journal of Biomechanics, 34(2):153-161, 2001.

[36] M. G. Pandy et al. An optimal control model for maximum-height human jumping. Journal of Biomechanics, 23(12):1185-1198, 1990.

[37] S. L. Delp et al. Opensim: open-source software to create and analyze dynamic simulations of movement. IEEE Transactions on Biomedical Engineering, 54(11):1940-1950, 2007.

[38] F. E. Zajac. Understanding muscle coordination of the human leg with dynamical simulations. Journal of Biomechanics, 35(8):1011-1018, 2002.

[39] J. A. Friederich and R. A. Brand. Muscle fiber architecture in the human lower limb. Journal of Biomechanics, 23(1):91-95, 1990.

[40] D. G. Lloyd and T. F. Besier. An emg-driven musculoskeletal model to estimate muscle forces and knee joint moments in vivo. Journal of Biomechanics, 36(6):765-776, 2003.

[41] D. G. Thelen. Adjustment of muscle mechanics model parameters to simulate dynamic contractions in older adults. Journal of Biomechanical Engineering, 125(1):70-77, 2003.

[42] M. Millard et al. Flexing computational muscle: modeling and simulation of musculotendon dynamics. Journal of Biomechanical Engineering, 135(2):021005, 2013.

[43] K. Manal and T. S. Buchanan. Modeling the non-linear relationship between emg and muscle activation. Journal of Biomechanics, 36:1197-1202, 2003.

[44] B. R. Umberger et al. A model of human muscle energy expenditure. Computer Methods in Biomechanics and Biomedical Engineering, 6(2):99-111, 2003.

[45] C. T. John. Complete description of the thelen2003muscle model.

[46] L. J. Bhargava et al. A phenomenological model for estimating metabolic energy consumption in muscle contraction. Journal of Biomechanics, 37(1):81-88, 2004.

[47] L. L. Menegaldo et al. Moment arms and musculotendon lengths estimation for a three-dimensional lower-limb model. Journal of Biomechanics, 37(9):1447-1453, 2004. 
[48] D. Hawkins and M. L. Hull. A method for determining lower extremity muscletendon lengths during flexion/extension movements. Journal of Biomechanics, 23(5):487-494, 1990.

[49] H. J. Hermens et al. European recommendations for surface electromyography. Roessingh Research and Development, 8(2):13-54, 1999.

[50] Stihii/Shutterstock Inc. Anatomical Body. Image used under license from shutterstock.com. http://www.shutterstock.com/pic-225637777. Accessed: 2016-09-22.

[51] MOOG FCS. 6DOF2000E Motion System User's Manual, 2008.

[52] NaturalPoint Inc. Arena Motion Capture Software Tutorial. www.optitrack.com, 2009.

[53] H. Tam and J. G. Webster. Minimizing electrode motion artifact by skin abrasion. Biomedical Engineering, IEEE Transactions on, (2):134-139, 1977.

[54] J. G. Webster. Reducing motion artifacts and interference in biopotential recording. Biomedical Engineering, IEEE Transactions on, (12):823-826, 1984.

[55] D. Farina et al. Influence of anatomical, physical, and detection-system parameters on surface emg. Biological Cybernetics, 86(6):445-456, 2002.

[56] EMG Sensors Product Sheet. Delsys Surface EMG Sensors. www.delsys.com, 2016.

[57] D. Luca et al. Inter-electrode spacing of surface emg sensors: reduction of crosstalk contamination during voluntary contractions. Journal of Biomechanics, 45(3):555-561, 2012.

[58] Delsys Inc. Delsys Bagnoli ${ }^{T M}$ EMG System User's Guide. www.delsys.com, 2014.

[59] D. M. Rouffet et al. Emg normalization to study muscle activation in cycling. Journal of Electromyography and Kinesiology, 18(5):866-878, 2008.

[60] Qubit Systems Inc. BBB1LP Breath by Breath Metabolic Analyzer. www.qubitsystems.com, 2009.

[61] N. J. Dahlkvist et al. Forces during squatting and rising from a deep squat. Engineering in Medicine, 11(2):69-76, 1982. 
[62] S. M. Henry et al. Control of stance during lateral and anterior/posterior surface translations. IEEE Transactions on Rehabilitation Engineering, 6(1):32-42, 1998.

[63] Kinovea. http://www.kinovea.org. Accessed: 2016-11-30.

[64] Simi Reality Motion Systems GmbH. Simi Shape 3D. http://www.simi.com Accessed: 2016-11-30.

[65] Great Lakes NeuroTechnologies. BioRadio. https://glneurotech.com/ bioradio/. Accessed: 2016-11-30.

[66] T. Xia and L. A. Frey-Law. A theoretical approach for modeling peripheral muscle fatigue and recovery. Journal of Biomechanics, 41(14):3046-3052, 2008.

[67] L. A. Frey-Law et al. A three-compartment muscle fatigue model accurately predicts joint-specific maximum endurance times for sustained isometric tasks. Journal of Biomechanics, 45(10):1803-1808, 2012. 


\section{Appendix A}

\section{Ethics Clearance}


Carleton

Canada's Capital University

\section{Ethics Clearance Form - New Clearance}

Carleton University

Research Ethics Office

Research Ethics Board

509C and 511 Tory, 1125 Colonel By Drive

Ottawa, ON K1S 5B6 Canada

Tel: 613-520-2517, ethics@carleton.ca

This is to certify that the Carleton University Research Ethics Board has examined the application for ethical clearance. The REB found the research project to meet appropriate ethical standards as outlined in the Tri-Council Policy Statement: Ethical Conduct for Research Involving Human, 2nd edition, and the Carleton University Policies and Procedures for the Ethical Conduct of Research.

Date of Clearance: October 08, 2014

Researcher: Burhanuddin Terai (Student Research: Master's Student)

Department: Faculty of Engineering and DesignlMechanical \& Aerospace Engineering (Department of) University: Carleton University

Research Supervisors (if applicable): Prof. Robert Langlois and Prof. Fred Afagh

Project Number: 102032

Alternate File Number (if applicable):

Project Title: Metabolic energy expenditure in maintaining postural stability during shipboard motions

Clearance Expires: May 31, 2015

\section{All researchers are governed by the following conditions:}

Annual Status Report: You are required to submit an Annual Status Report to either renew clearance or close the file. Failure to submit the Annual Status Report will result in the immediate suspension of the project. Funded projects will have accounts suspended until the report is submitted and approved.

Changes to the project: Any changes to the project must be submitted to the Carleton University Research Ethics Board for approval. All changes must be approved prior to the continuance of the research.

Adverse events: Should a participant suffer adversely from their participation in the project you are required to report the matter to the Carleton University Research Ethics Board. You must submit a written record of the event and indicate what steps you have taken to resolve the situation.

Suspension or termination of clearance: Failure to conduct the research in accordance with the principles of the Tri-Council Policy Statement: Ethical Conduct for Research Involving Humans, 2nd edition and the Carleton University Policies and Procedures for the Ethical Conduct of Research may result in the suspension or termination of the research project.

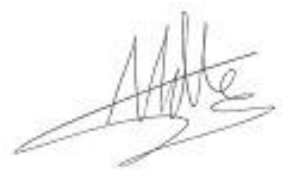

Andy Adler

Chair, Carleton University Research Ethics Board

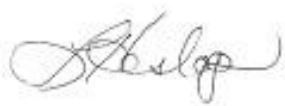

Louise Heslop

Vice-Chair, Carleton University Research Ethics Board 
Appendix B

Sample Joint Torque Profiles 

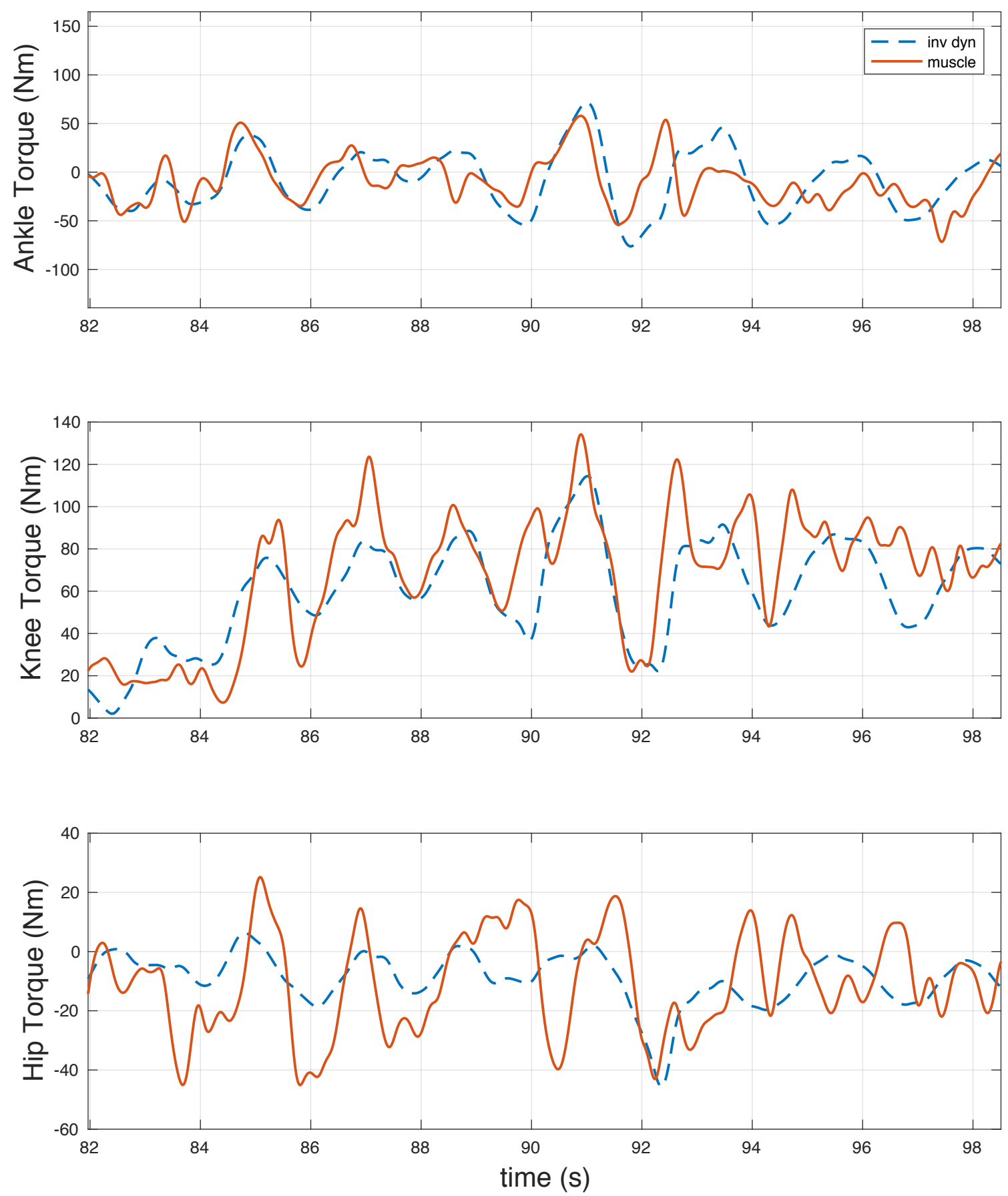

Figure B.1: Subject 1 joint torques from MTM muscle model and 3IP inverse dynamics during a combined 3DOF motion profile. 

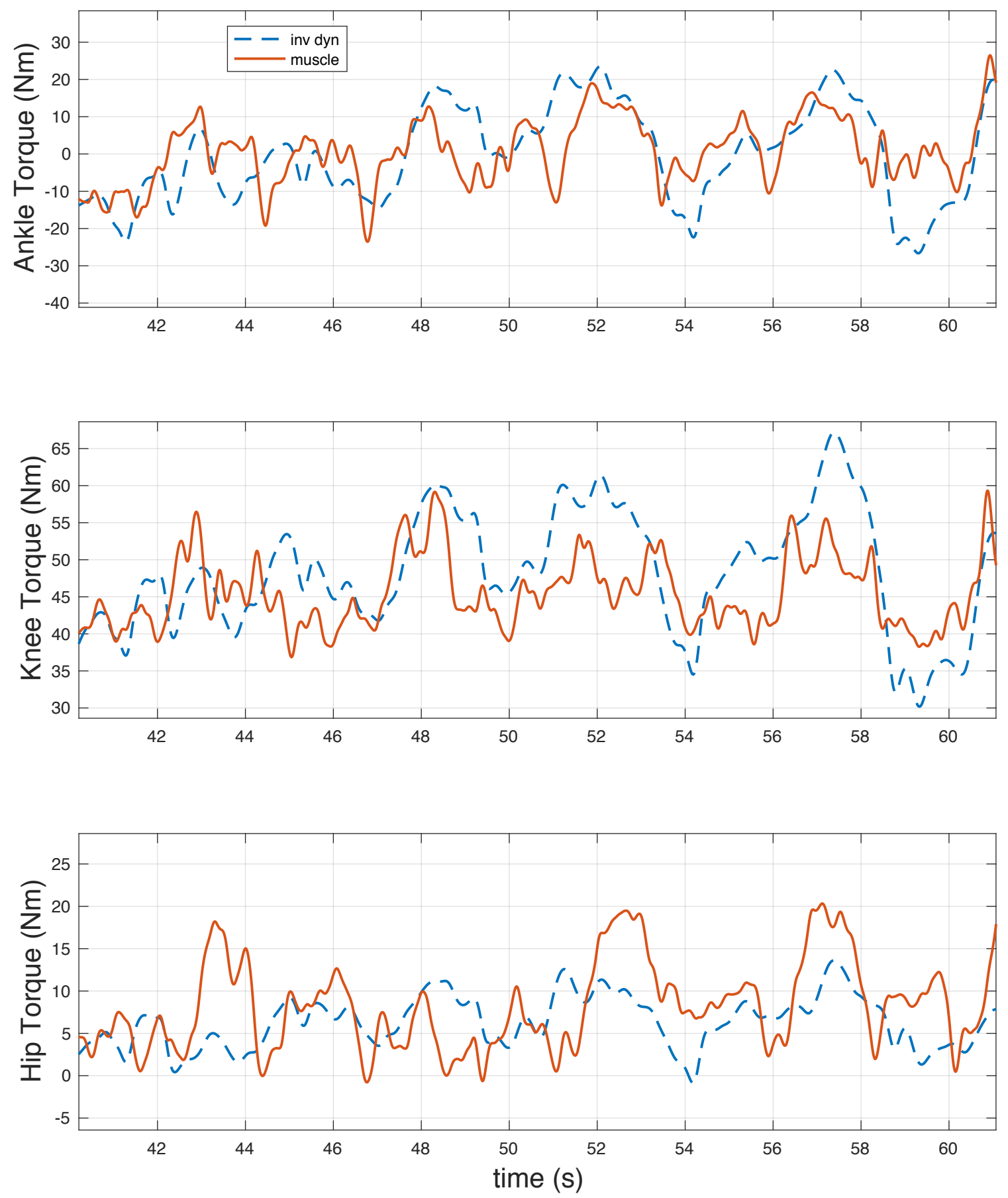

Figure B.2: Subject 2 joint torques from MTM muscle model and 3IP inverse dynamics during a combined 3DOF motion profile. 

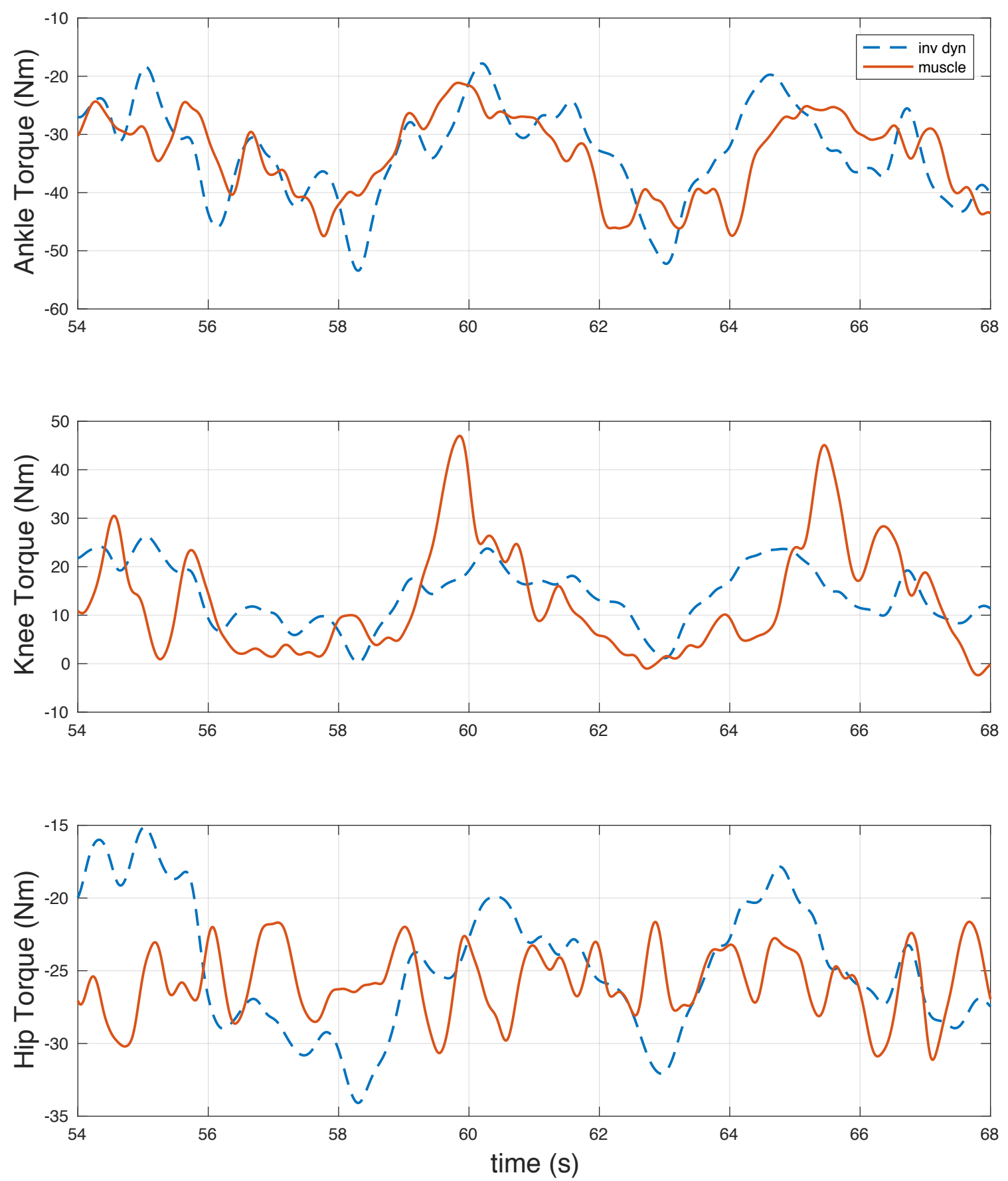

Figure B.3: Subject 3 joint torques from MTM muscle model and 3IP inverse dynamics during a surge chirp profile. 

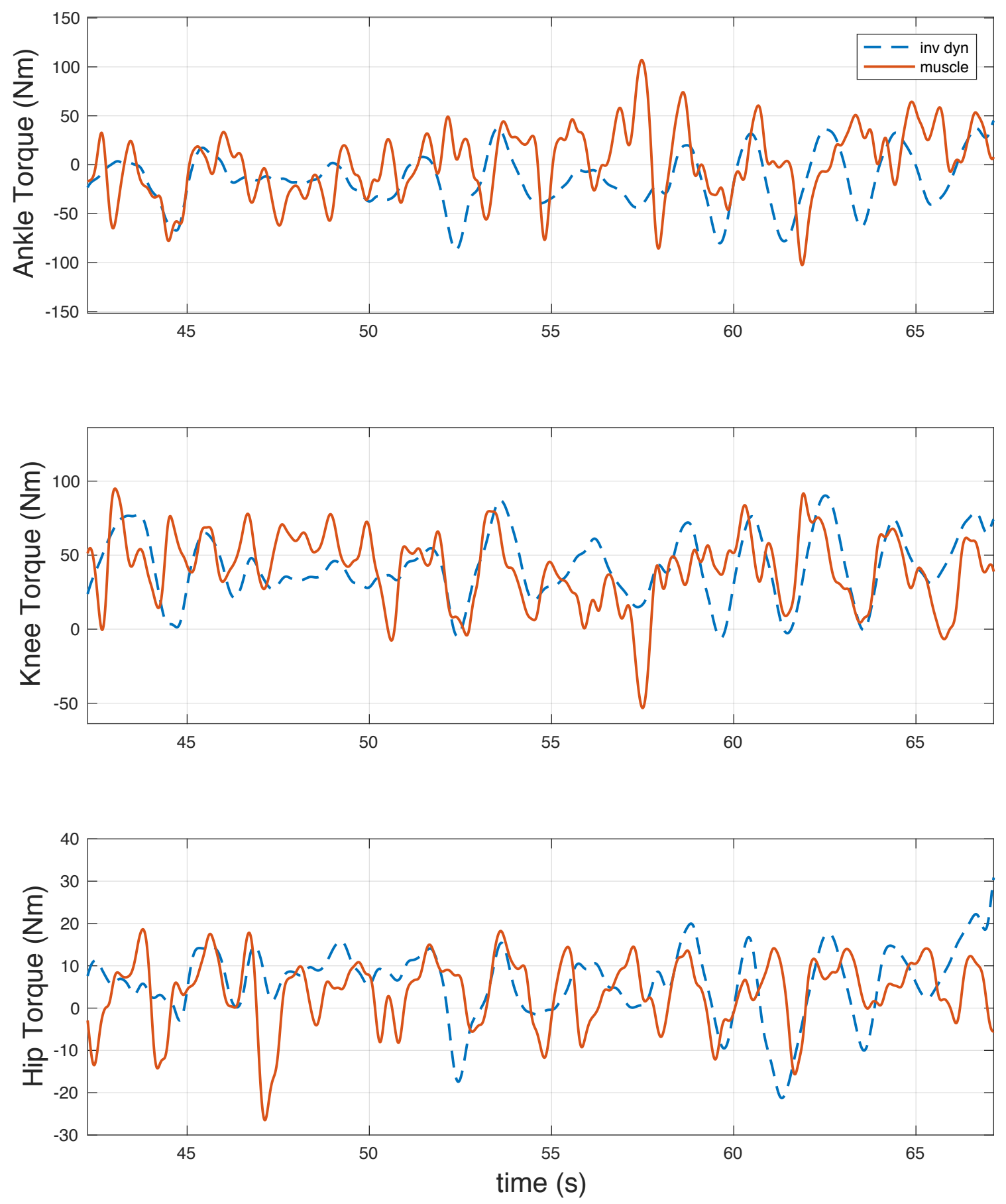

Figure B.4: Subject 4 joint torques from MTM muscle model and 3IP inverse dynamics during a combined 3DOF motion profile. 

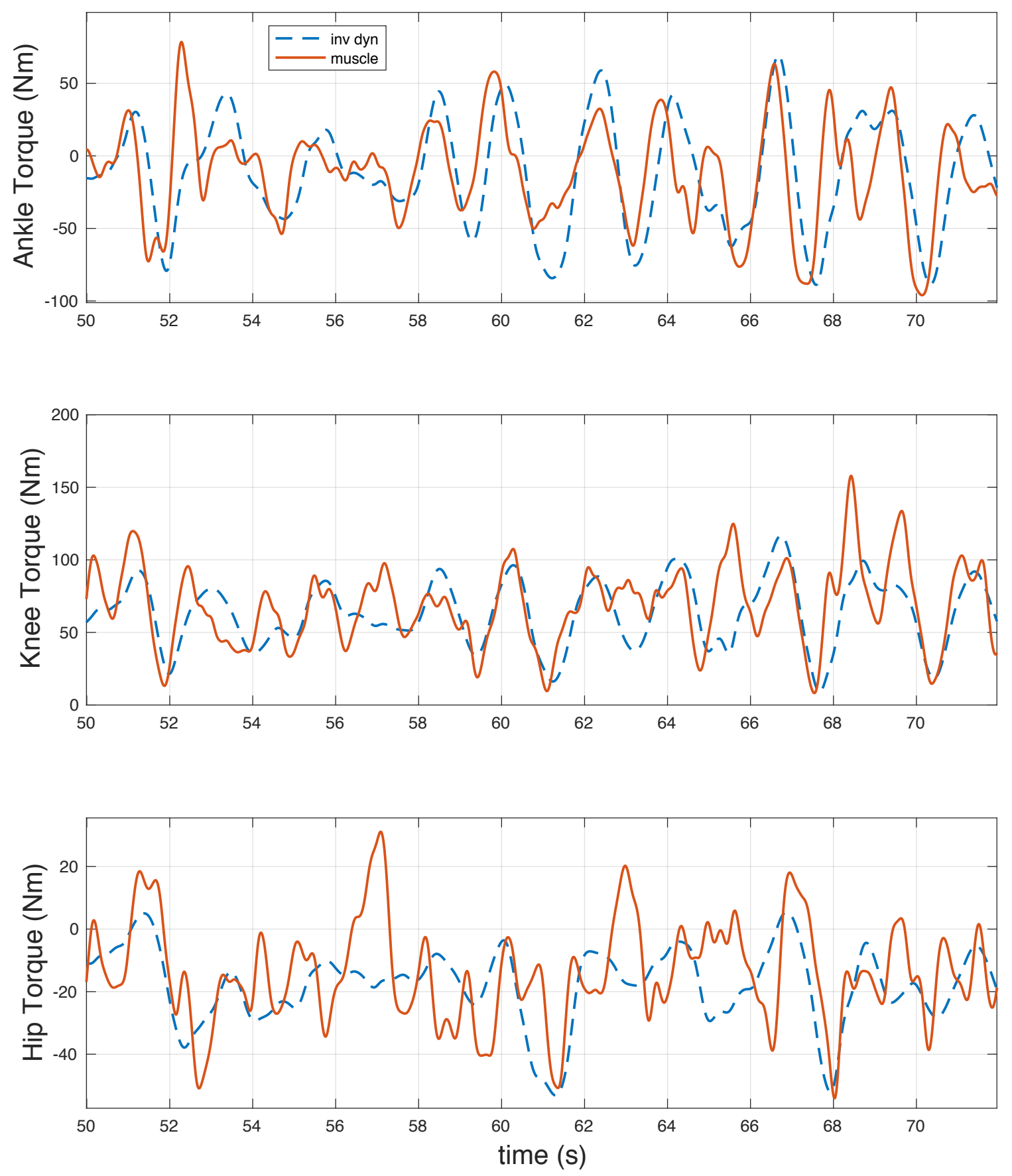

Figure B.5: Subject 5 joint torques from MTM muscle model and 3IP inverse dynamics during a combined 3DOF motion profile. 

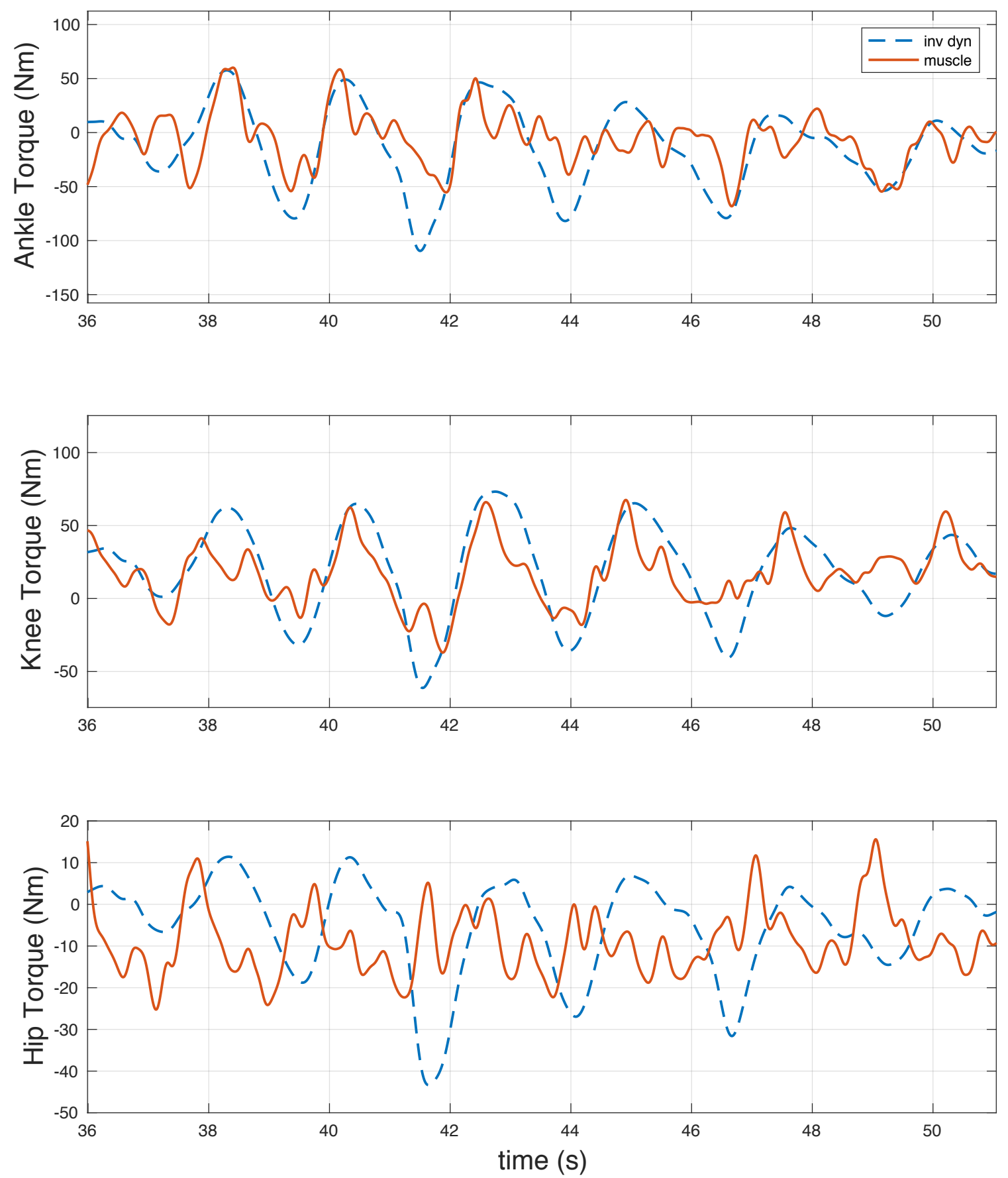

Figure B.6: Subject 6 joint torques from MTM muscle model and 3IP inverse dynamics during a combined 3DOF motion profile. 

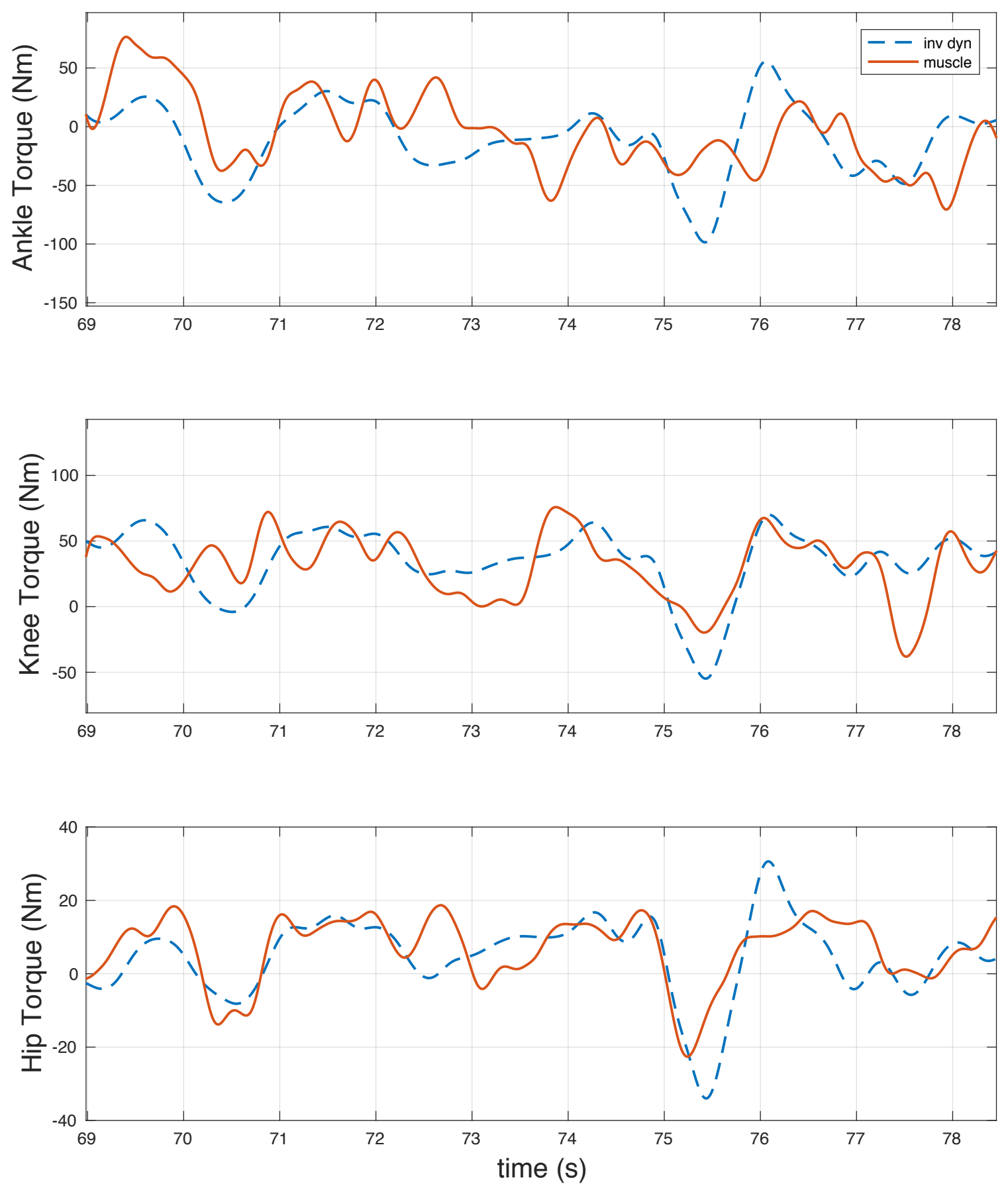

Figure B.7: Subject 7 joint torques from MTM muscle model and 3IP inverse dynamics during a combined $3 \mathrm{DOF}$ motion profile. 